\title{
Differential Equations for Machine Learning
}

Çağatay Yıldız

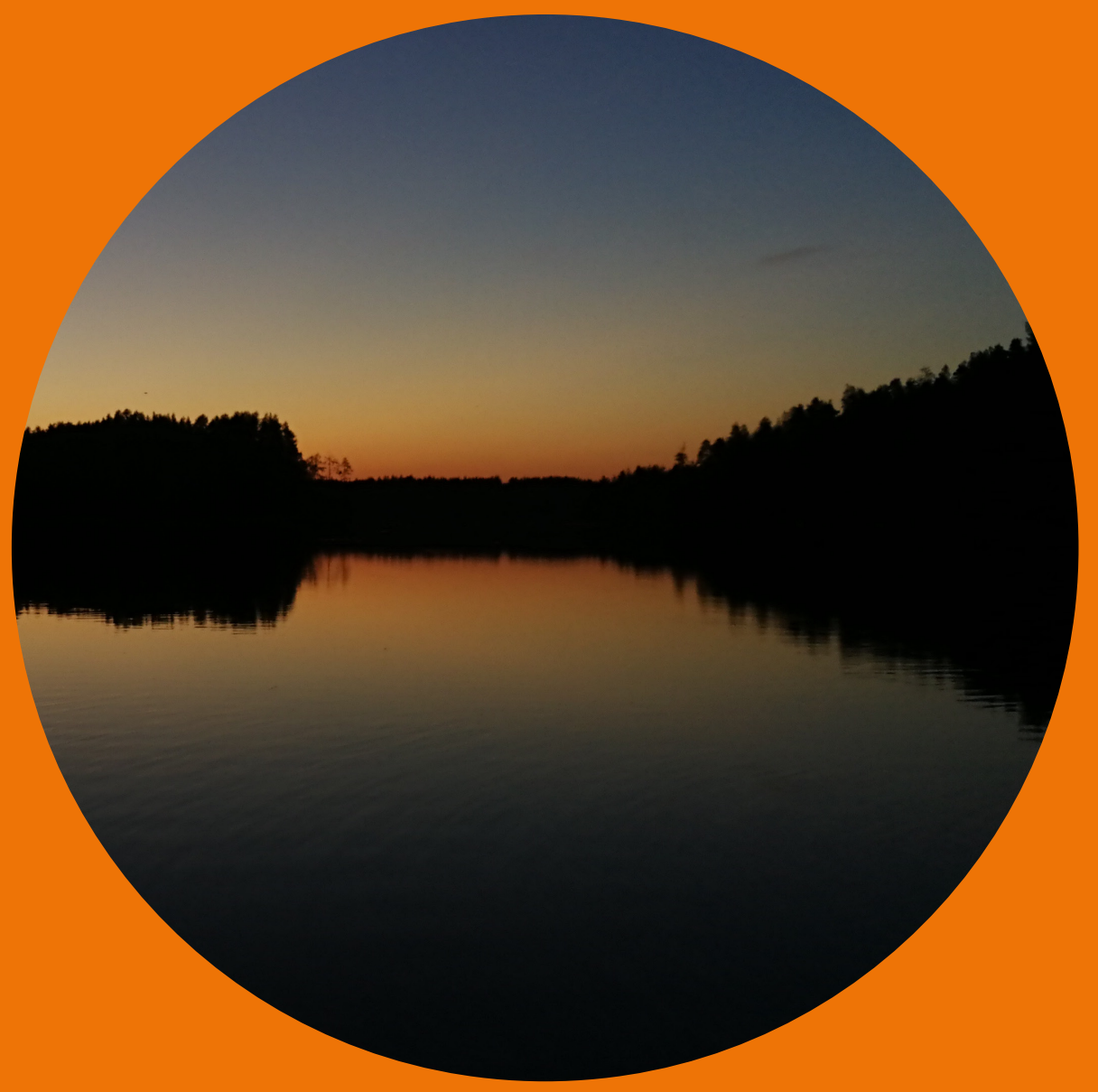




\section{Differential Equations for Machine Learning}

Çağatay Yıldız

A doctoral dissertation completed for the degree of Doctor of Science (Technology) to be defended, with the permission of the Aalto University School of Science, https://aalto.zoom.us/j/61873808631, on 18 February 2022 at 12:15. 
Professor Harri Lähdesmäki, Aalto University, Finland

\section{Thesis advisors}

Professor Harri Lähdesmäki, Aalto University, Finland

Doctor Markus Heinonen, Aalto University, Finland

\section{Preliminary examiners}

Professor Arto Klami, University of Helsinki, Finland

Professor Niklas Wahlström, Uppsala University, Sweden

\section{Opponent}

Professor Carl Henrik Ek, University of Cambridge, United Kingdom

DOCTORAL THESES $7 / 2022$

\section{(C) 2022 Çağatay Yıldız}

ISBN 978-952-64-0665-7 (printed)

ISBN 978-952-64-0666-4 (pdf)

ISSN 1799-4934 (printed)

ISSN 1799-4942 (pdf)

http://urn.fi/URN:ISBN:978-952-64-0666-4

Unigrafia Oy

Helsinki 2022

Finland 
Author

Çağatay Yıldız

Name of the doctoral thesis

Differential Equations for Machine Learning

\begin{tabular}{|c|c|}
\hline \multicolumn{2}{|l|}{ Publisher School of Science } \\
\hline \multicolumn{2}{|l|}{ Unit Department of Computer Science } \\
\hline \multicolumn{2}{|l|}{ Series Aalto University publication series DOCTORAL THESES 7/2022 } \\
\hline \multicolumn{2}{|l|}{ Field of research Machine Learning } \\
\hline Manuscript submitted 15 June 2021 & ce 18 February 2022 \\
\hline Permission for public defence granted (date) 17 November $2021 \quad$ Language English & Language English \\
\hline$\bigotimes$ Article thesis & $\square$ Essay thesis \\
\hline
\end{tabular}

Abstract

Mechanistic models express novel hypotheses for an observed phenomenon by constructing mathematical formulations of causal mechanisms. As opposed to this modeling paradigm, machine learning approaches learn input-output mappings by complicated and often non-interpretable models. While requiring large chunks of data for successful training and downstream performance, the resulting models can come with universal approximation guarantees. Historically, differential equations (DEs) developed in physics, economics, engineering, and numerous other fields have relied on the principles of mechanistic modeling. Despite providing causality and interpretability that machine learning approaches usually lack, mechanistic differential equation models tend to carry oversimplified assumptions. In this dissertation, we aim to bring these two worlds together by demonstrating how machine learning problems can be tackled by means of differential equations, and how differential equation models can benefit from modern machine learning tools.

First, we examine the problems in which mechanistic modeling becomes too difficult, which include the cases with partial knowledge about the observed system and with an excessive number of interactions. Such limitations complicate the process of constructing mathematical descriptions of the phenomenon of interest. To bypass this, we propose to place Gaussian process priors to the time differential and diffusion functions of unknown ordinary (ODEs) and stochastic differential equations (SDEs), and approximate the resulting intractable posterior distribution. We demonstrate that the model can estimate unknown dynamics from sparse and noisy observations.

Motivated by the fact that our proposed approach is unable to learn sequences obtained by transforming the ODE states, we develop a new technique that can simultaneously embed the observations into a latent space, and learn an ODE system in the embedding space. Our new model infers the dynamics using Bayesian neural networks for uncertainty handling and more expressive power. We furthermore explicitly decompose the latent space into momentum and position components, which leads to increased predictive performance on a variety of physical tasks.

Our next task concerns another problem involving DEs, namely, non-convex optimization. By carefully crafting the drift and diffusion functions of an SDE, we first obtain a stochastic gradient MCMC algorithm. Tuning a temperature variable in the proposed algorithm allows the chain to converge to the global minimum of a non-convex loss surface. We significantly speed up the convergence by using second-order Hessian information in an asynchronous parallel framework.

Lastly, we explore how reinforcement learning problems can benefit from neural network based ODE models. In particular, we propose to learn dynamical systems controlled by external actions by a novel, uncertainty-aware neural ODE model. The inferred model, in turn, is utilized for learning optimal policy functions. We illustrate that our method is robust to both noisy and irregularly sampled data sequences, which poses major challenges to traditional methods.

Keywords Machine learning, differential equations, neural networks, Gaussian processes

\begin{tabular}{lc}
\hline ISBN (printed) $978-952-64-0665-7$ & ISBN (pdf) $978-952-64-0666-4$ \\
\hline ISSN (printed) $1799-4934$ & ISSN (pdf) $1799-4942$ \\
\hline Location of publisher Helsinki & Location of printing Helsinki $\quad$ Year 2022 \\
\hline Pages 178 & urn http://urn.fi/URN:ISBN:978-952-64-0666-4 \\
\hline
\end{tabular}





\section{Preface}

This journey started when Taha asked me about applying to Aalto University, a place that I had barely heard about. The second northernmost capital in the world did not seem like the perfect destination at first but that quickly changed after contacting Harri Lähdesmäki. Looking back four years later, I could not have asked for a better supervisor. Your guidance and support have helped me (who knows how many times) find my way through seemingly impossible-to-escape-from situations. I will truly miss those eureka moments I had during our discussions. Thank you for showing me research is enjoyable and letting me develop myself as an independent researcher.

Next, I would like to thank Markus Heinonen for his immense support throughout my journey. Our day-to-day, sometimes hours-long conversations helped me leap over countless obstacles. I have learned so much from you, not just the theory but also how to ask questions and conduct research. I am grateful particularly for your patience in my early times, answering all my questions regardless of how silly they are.

I would also like to express my gratitude to many colleagues, starting with my coauthors Henrik Mannerström and Jukka Intosalmi. My first two publications would not be possible without your ideas and contributions, particularly the sensitivity equations. Thanks Pashupati Hegde for our ODE discussions and code exchanges. Charles Gadd, our regular RL whinings assisted me to gain an understanding of the whole discipline and kept me going; thanks for sharing those moments with me. It has really been a privilege to brainstorm with excellent researchers at Aalto; in particular, Alexander Ilin, Arno Solin, and Ville Kyrki. Thank you Umut Şimşekli for your supervision and lead in our asynchronous LBFGS project; you showed me how rewarding such difficult projects can be. Thomas Schön, I am very thankful to have visited your group through the end of my doctoral studies. Finally, I would like to thank the pre-examiners Arto Klami and Niklas Wahlström for their valuable feedback.

In my years at the Computational Systems Biology research group, I have been lucky enough to meet many great colleagues. Juho Timonen, 
Maria Osmala, Siddharth Ramchandran, Sini Rautio, Emmi Jokinen, Viivi Halla-aho, Juhi Somani, Lu Cheng, Valerii Iakovlev, and Gleb Tikhonov; thanks for creating this inspiring and supportive work environment, fun conference trips, lunches, and after-work beers. Thank you Betül Güvenç, Tolou Shadbahr, Yogesh Kumar, Santosh Hiremath, Kunal Ghosh, Eero Siivola, Pradeep Eranti, Tianyu Cui, Topi Paananen, Reza Ashrafi and other fellow PhD students not mentioned here for occasional, stimulating chats. Aija Kukkala, Noora Suominen de Rios, Richard Darst, Jaakko Kotimäki, Simo Tuomisto and other members of the administrative and Science-IT team, I am thankful for your support with all my daily issues.

Mine Öğretir and Onur Poyraz; my life in Finland and PhD experience changed for the better right upon your arrival. I count myself very fortunate to have your company, especially through this spring. Çağlar Hızlı and Ashutosh Vaishnav; getting you on board was also lovely, I will surely miss our suddenly but comfortably deep conversations. Daniel MonsivaisVelazquez; having you as my first flatmate and friend here was gratifying; I will definitely keep alive the memory of your awkwardly funny jokes. My dear friends Taha Ceritli, Berkant Kepez, Emre Erdoğan, Barış Kurt, Beyza Ermiş, Şebnem Gelmedi, and also the other member of the "Börekçi" team, Muhammed Tel, Tuna Kırabalı, Orkun Uçkunlar, Ahmet Duran Arslan, and Ümit Yapıcı; those little get-together breaks, endless remote talks and football chitchats animated me a zillion times. Thank you all for adding so much color to my life.

Finally, I wholeheartedly thank my family for their never-ending encouragement, unconditional love and support in my endeavour to become a scientist, for which I will always be indebted. Dear Tuğçe Çetin; meeting you has completely turned my life around. Thank you for constant presence, providing me with the safest possible environment to explore my own map and open new doors no matter how scary they are.

Helsinki, December 27, 2021,

Çağatay Yıldız 


\section{Contents}

Preface

$\begin{array}{lll}\text { Contents } & \text { iii }\end{array}$

List of Publications vii

$\begin{array}{ll}\text { Author's Contribution } & \text { ix }\end{array}$

Abbreviations $\quad$ xi

1. Introduction 1

1.1 Contributions . . . . . . . . . . . . . 2

1.2 Structure of the Dissertation . . . . . . . . . . . . 4

2. Theoretical Foundations $\mathbf{5}$

2.1 Function Approximation _............ . . 6

2.1.1 Neural Networks . . . . . . . . . . 6

2.1.2 Gaussian Processes . . . . . . . . . . . 7

2.2 Differential Equations . . . . . . . . . . . . . 9

2.2.1 Ordinary Differential Equations . . . . . . 9

2.2.2 Stochastic Differential Equations . . . . . . . 10

2.2.3 Numerical Solvers . . . . . . . . . . . . . . . 12

2.2.4 Gradient Computation ........... 15

2.3 A Primer on Parameter Inference . . . . . . . . . . . . . 17

2.3.1 Variational Inference . . . . . . . . . . . . 18

2.3.2 Stochastic Gradient Markov Chain Monte Carlo 21

2.3.3 Numerical Optimization ... . . . . . . 23

3. Gaussian Processes for Learning Unknown Differential Equations $\quad 25$

3.1 Auto-Regressive Dynamics Learning . . . . . . . . . . 26

3.1.1 Gradient Matching . . . . . . . . . . . 26

3.1.2 Related Work . . . . . . . . . . . . . . 28 
3.2 Learning ODEs . . . . . . . . . . . . . . . . . 28

3.2.1 Time Differential Function . . . . . . . . . . 29

3.2.2 MAP Inference for npODE . . . . . . . . . . . 29

3.3 Learning SDEs . . . . . . . . . . . . . . . . . . . . . 31

3.4 Experiments with npODE and npSDE ........ 34

3.4.1 Simulated ODE Systems . . . . . . . . . . 35

3.4.2 Simulated SDE System . . . . . . . . . . . 35

3.4.3 Motion Capture Experiments . . . . . . . 36

4. Continuous-Time Bayesian Modelling for High Dimensional Sequences $\quad 39$

4.1 Limitations of $n$ pODE . . . . . . . . . . . . . . . . 39

4.1.1 Modeling Space . . . . . . . . . . . . 39

4.1.2 Learning Second-Order Systems . . . . . . . . 40

4.1.3 Scalability of the Dynamics . . . . . . . . 40

4.1 .4 Overfitting ................ 41

4.1.5 Scalability of the Optimization . . . . . . . 41

4.2 Proposed Model . . . . . . . . . . . . . . . . . 41

4.2.1 Approximate Posterior . . . . . . . . . . 43

4.2 .2 Evidence Lower Bound . . . . . . . . . . . . 44

4.3 Experiments . . . . . . . . . . . . . . 46

4.3.1 Robustness against Overfitting . . . . . . . . 47

4.3.2 Generalization to Unseen Data Points . . . . . . 47

4.3.3 The Virtue of Second-Order Modeling . . . . . . 48

5. SDEs for Stochastic Asynchronous Non-convex Optimization $\mathbf{5 1}$

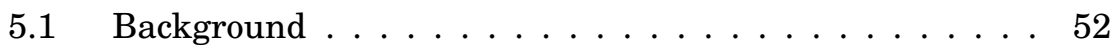

5.1 .1 Stochastic L-BFGS . . . . . . . . . . . . . 53

5.1 .2 Tempered SGLD . . . . . . . . . . . . . 53

5.2 Asynchronous Stochastic L-BFGS . . . . . . . . . . . . 54

5.2.1 Designing the Correct SDE . . . . . . . . . 54

5.2.2 A Practical Distributed Algorithm . . . . . . 55

5.2 .3 Main Theoretical Result . . . . . . . . . 56

5.3 Large-scale Matrix Factorization Experiment . . . . . . . 57

6. Continuous-Time Model-Based Reinforcement Learning 61

6.1 Problem Formulation . . . . . . . . . . . . . 62

6.2 Dynamics Learning . . . . . . . . . . . . . . 63

6.3 Continuous-time Actor-Critic . . . . . . . . . . 65

6.3.1 State-Value Approximation (Critic) . . . . . . 66

6.3.2 Estimating the Actor and Critic . . . . . . . . 67

6.3.3 Connection to Temporal Difference . . . . . . 67

6.4 Previous Work ................. 68

6.5 A New CTRL Evaluation Environment . . . . . . . . . 69 
6.6 Experimental Results .............. 70

$\begin{array}{ll}\text { 7. Conclusion and Future Directions } & \mathbf{7 3}\end{array}$

$\begin{array}{ll}\text { References } & \mathbf{7 7}\end{array}$

$\begin{array}{ll}\text { Publications } & \mathbf{8 9}\end{array}$ 



\section{List of Publications}

This thesis consists of an overview and of the following publications which are referred to in the text by their Roman numerals.

I Markus Heinonen*, Çăgatay Yıldız*, Henrik Mannerström, Jukka Intosalmi, Harri Lähdesmäki. Learning Unknown ODE Models with Gaussian Processes. In International Conference on Machine Learning, Stockholm, pages 1959-1968, vol.80, July 2018.

II Çağatay Yıldız, Markus Heinonen, Jukka Intosalmi, Henrik Mannerström, Harri Lähdesmäki. Learning Stochastic Differential Equations with Gaussian Processes without Gradient Matching. In 2018 IEEE 28th International Workshop on Machine Learning for Signal Processing (MLSP), Aalborg, doi=10.1109/ MLSP.2018.8516991. (C) 2018 IEEE. Reprinted, with permission, from the authors, September 2018.

III Çağatay Yıldız, Markus Heinonen, Harri Lähdesmäki. ODE ${ }^{2}$ VAE: Deep Generative Second Order ODEs with Bayesian Neural Networks. In Neural Information Processing Systems, Vancouver, pages 13412-13421, vol.32, December 2019.

IV Umut Şimşekli, Çağatay Yıldız, Than Huy Nguyen, Taylan Cemgil and Gael Richard. Asynchronous Stochastic Quasi-Newton MCMC for Non-Convex Optimization. In International Conference on Machine Learning, Stockholm, pages 4674-4683, vol.80, July 2018.

V Çağatay Yıldız, Markus Heinonen, Harri Lähdesmäki. ContinuousTime Model-Based Reinforcement Learning. In International Conference on Machine Learning, Virtual, pages 12009-12018, vol.139, July 2021. 



\section{Author's Contribution}

\section{Publication I: "Learning Unknown ODE Models with Gaussian Processes"}

The initial idea of Gaussian process based ODE modeling was proposed by Markus Heinonen and Harri Lähdesmäki. The final version of the model was decided by discussions including all authors. Jukka Intosalmi suggested using sensitivity equations and provided an example code. I improved the toy model implemented by Markus Heinonen, and unified it with sensitivity equations. I performed all the experiments, which were designed by Markus and myself. The article was written by all authors.

\section{Publication II: "Learning Stochastic Differential Equations with Gaussian Processes without Gradient Matching"}

The idea of extending our previous method to SDEs was discussed among all authors. Markus Heinonen and I worked on MATLAB and TensorFlow implementations in parallel. I designed and executed the experiments. Markus Heinonen, Harri Lähdesmäki and I wrote the publication while other coauthors helped us with proofreading and corrections.

\section{Publication III: "ODE²VAE: Deep Generative Second Order ODEs with Bayesian Neural Networks"}

The original idea was proposed by Markus Heinonen and Harri Lähdesmäki. I performed the literature survey, derived the theoretical aspects, implemented the method, designed and executed the experiments. All authors jointly wrote the article. 


\section{Publication IV: “Asynchronous Stochastic Quasi-Newton MCMC for Non-Convex Optimization"}

Umut Şimşekli came up with the idea of building an asynchronous L-BFGS method. Umut Şimşekli and I discussed the underlying mathematical construction and experiments. I developed the main asynchronous algorithm while Umut Şimşekli held the responsibility of writing the article and proving our theorems.

\section{Publication V: "Continuous-Time Model-Based Reinforcement Learning"}

Markus Heinonen and Harri Lähdesmäki proposed the idea of building a continuous-time reinforcement learning method. In conjunction with our discussions, I developed new variants of the standard continuous-time approach and derived a novel policy learning method. I implemented the method and executed all the experiments. The article was written jointly. 


\section{Abbreviations}

ACA adaptive checkpoint algorithm

AR auto-regressive models

a-SGD asynchronous SGD

BFGS Broyden-Fletcher-Goldfarb-Shanno

BNN Bayesian neural network

CNN convolutional neural network

CT continuous-time

CTRL continuous-time reinforcement learning

DT discrete-time

EM Euler-Maruyama

GP Gaussian process

HAMCMC Hessian approximated MCMC

HMC Hamiltonian Monte Carlo

iid independently and identically distributed

KL Kullback-Leibler

L-BFGS limited memory BFGS

MAP maximum a posteriori

mb-L-BFGS multi-batch LBFGS

MBRL model-based reinforcement learning

MCMC Markov chain Monte Carlo 
ML maximum likelihood

MLP multi-layer perceptron

MSE mean squared error

NN neural network

NODE neural ODE

NPETS non-linear PETS

npODE non-parametric ODE

npSDE non-parametric SDE

ODE ordinary differential equation

PCA principle component analysis

PETS probabilistic ensembles with trajectory sampling

PILCO probabilistic inference for learning control

$\mathbf{R B F}$ radial basis function

RL reinforcement learning

RMSE root mean squared error

RNN recurrent neural network

SDE stochastic differential equation

SGD stochastic gradient descent

SGLD stochastic gradient Langevin dynamics

SGMCMC stochastic gradient MCMC

SGP sparse Gaussian process

VAE variational auto-encoder 


\section{Introduction}

Time series forecasting has been one of the core problems in statistics and machine learning. In the most basic form, the goal is to make predictions based on historical measurements, which typically requires learning how the measured variables evolve over time (Barber et al., 2011; Durbin and Koopman, 2012; Särkkä, 2013). For decades, researchers have addressed this problem by discrete dynamical systems, where the variable of interest jumps from one value to another as the system moves from "one point in time" to the other. Perhaps the most well-known example of such discrete dynamical systems is the Kalman filter (Kalman, 1960), which has been successfully applied in a wide range of fields (Barber et al., 2011). Nevertheless, variables in certain problems such as radioactive decay, the flow of current in a conductor and the growth of a population necessitate treating time as a continuum, meaning that the variables can only have a particular value for only an infinitesimally short amount of time. Such problems are broadly referred to as continuous-time problems.

Since discrete dynamical systems are rather straightforward to grasp and implement, they have been often applied to continuous-time problems (Barber et al., 2011; Murphy, 2013). This is also nicely complemented by the fact that continuous signals in modern day computers are almost always represented in a discrete manner, e.g., by a finite collection of samples. However, as demonstrated later in this dissertation, the success of discrete systems on truly continuous-time problems is restricted by factors such as high sampling frequency and low measurement noise. This natural mismatch can be addressed by turning to differential equations, which can be defined as equations that involve functions and their derivatives (Tenenbaum, 1985). Dating back to the discovery of calculus by Leibniz and Newton, differential equations were first used to explain the laws of motion and have played a vital role in many physical theories ever since (see Robinson (2004) for a discussion).

Ordinary differential equations (ODEs) form a special subgroup of differential equations in which the unknown function depends on a single independent variable. When an ODE involves a stochastic process, the 
resulting system is referred to as stochastic differential equation (SDE). We focus our attention on ODEs and SDEs, without loss of generality, with the independent variable being time; hence, differential equation systems of our interest describe the time evolution of dependent variables. In essence, our goal is to build mathematical models for the functions appearing in such differential equation systems. Accurate models can then be used for predicting the system's future behavior.

This problem has been studied for decades in the contexts of dynamical systems learning and system identification. Conventional ODE approaches relying on white-box modeling assume that a parametric model for the process of interest is given (Ljung, 2010). Consequently, the ODE inference problem reduces to parameter estimation, which can be tackled using optimization and statistical inference tools (Butcher and Goodwin, 2008). We refer to the methods following this traditional ODE modeling approach as "parametric (ODE) models".

Successful applications of parametric ODE models necessitate accurate functional forms describing the system dynamics. Nonetheless, researchers often have partial knowledge about the dynamics which leads to an incomplete representation of observed phenomena. Similarly, formulating complex dynamics directly from data that possibly consist of hundreds of observations, is a tedious task, and parametric models may only provide too simplistic explanations (Äijö and Lähdesmäki, 2009). Therefore, accurate descriptions of the real-world system dynamics could easily become an impractical task, if not impossible.

This dissertation concerns an alternative approach to conventional techniques, namely, non-parametric, or black-box, ODE modeling. Leveraging the advances in function estimators, we propose to infer unknown system dynamics with Gaussian processes (GP) or neural networks (NN). Thanks to the universal function approximation theorems (Cybenko, 1989; Hammer and Gersmann, 2003), the resulting black-box ODE systems would theoretically be able to learn unknown differential equations from arbitrary data sequences. We experimentally demonstrate that the proposed models can indeed learn a variety of dynamical systems, even from image sequences or with external control signals.

\subsection{Contributions}

Before explaining the proposed approaches in detail, we give a general overview of the research questions this dissertation addresses and the solutions devised. Please see Figure 1.1 for a schematic illustration of how the developed methods are related.

- Publication I presents $n p O D E$, our first attempt to model unknown ODE systems in a black-box manner. Due to their inherent ability 


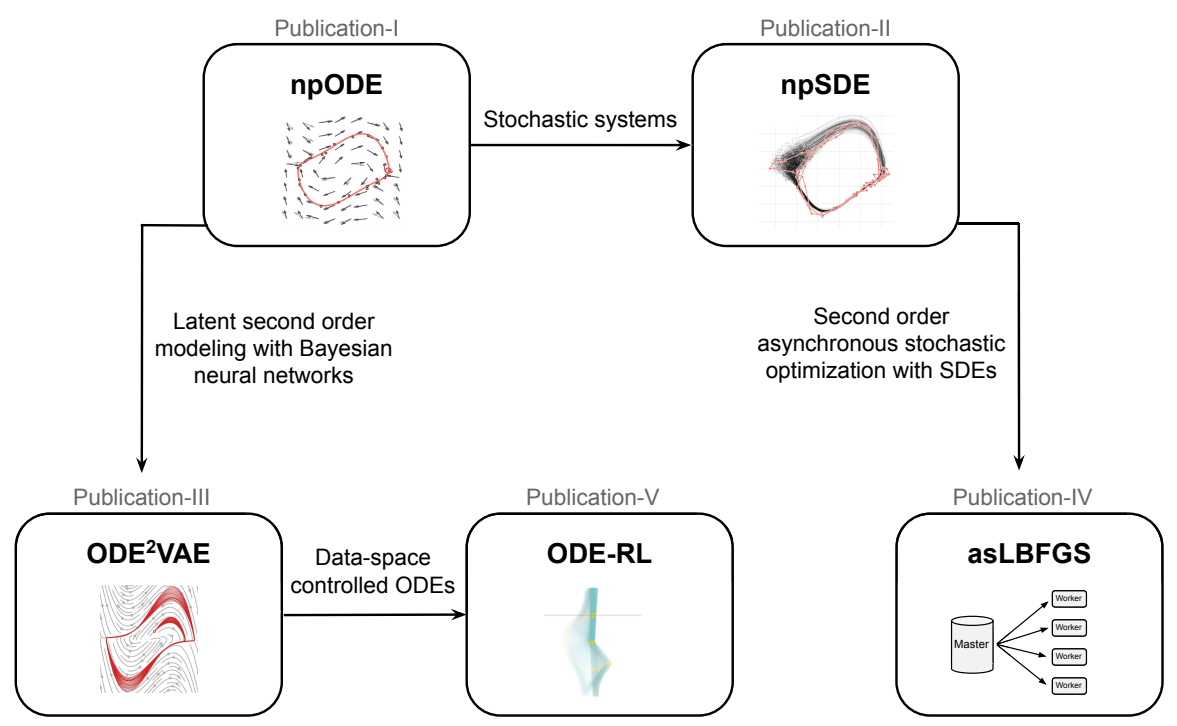

Figure 1.1. A diagram of the methods presented in this dissertation. We first develop a black-box ODE model based on Gaussian processes, which is later extended to an SDE model. Next we investigate how SDE formulations can be used for large-scale optimization (Publication IV). Publication III describes a technique to learn ODEs from video sequences. Finally, Publication V applies black-box ODE modelling approach to reinforcement learning problems.

to learn smooth function, we opt for inducing point approximations of GPs to approximate the unknown time differentials. To efficiently compute the gradients of the system, we propose to use another set of differential equations, named sensitivity equations. We demonstrated the model's ability to learn arbitrary systems on three different parametric ODE systems without seeing the functional forms as well as the dynamics of real walking sequences.

- The above technique is adapted to SDEs in Publication II (hence called $n p S D E$ ). Our methodology is based on simulating path distributions by forward integration to match observed sequences. We also propose a practical, sampling-based likelihood model in connection with kernel density estimation. Experimental evaluations demonstrate that arbitrary drift functions as well as state-dependent and constant diffusion functions can be accurately estimated.

- Kernel methods, including GPs, tend to suffer from curse of dimensionality (Evangelista et al., 2006), which limits the applicability of our previous GP-based ODE and SDE approaches to small and medium dimensional problems. Motivated by the fact that NNs excel at high-dimensional tasks such as high-resolution image generation and classification (LeCun et al., 2015), we propose to approximate unknown differentials with Bayesian neural networks (BNN) to ac- 
count for scalability as well as uncertainty. Furthermore, we describe how to embed high dimensional trajectories, such as video sequences, into a low dimensional latent space in which ODE system is defined. We also explicitly decompose the latent space into momentum and position components, and name our method $O D E^{2} V A E$ (Publication III).

- In Publication IV, we shift our attention from unknown differential equations to numerical optimization. Our technique, named as-LBFGS, relies on the numerical simulation of a specific type of SDE known as Langevin dynamics. We first show that the dynamics, when coupled with an additional temperature parameter, can be used to reach the global optimum of a target function. Furthermore, we propose to include local geometric information through an approximation to the Hessian matrix and show how to perform the optimization procedure in an asynchronous manner. The experiments show that our algorithm provides a significant speedup over existing synchronous distributed algorithms.

- The majority of physical systems and control tasks are described in form of differential equations; however, existing reinforcement techniques are inherently designed for discrete setups. We address this mismatch in Publication $\mathrm{V}$ by introducing $O D E-R L$, a continuoustime reinforcement learning (RL) algorithm. Similar to Publication III, we propose a BNN-based ODE model that involve control signals, and also develop a novel continuous-time actor-critic method to learn optimal policies. We demonstrate that our method is robust to both noisy and irregularly sampled data sequences, which poses major challenges to traditional discrete-time methods.

\subsection{Structure of the Dissertation}

The next chapter outlines the technical background needed to understand the remaining of the dissertation. Chapter 3 presents our GP-based ODE and SDE methods (Publication I-II). ODE ${ }^{2}$ VAE is described in the following chapter. We then explain as-LBFGS in Chapter 5 and Chapter 6 is devoted to ODE-RL. In each chapter, we give a summary of the proposed methods and experimental findings. Hence, readers can study each chapter separately after related technical background is surveyed. The dissertation is concluded with a discussion of the presented techniques and future directions. 


\section{Theoretical Foundations}

In this chapter, we briefly explain the concepts on which our proposed models are built:

- The first section gives an overview of function approximation in machine learning. In particular, we discuss Gaussian processes and different types of neural networks.

- Our Publication I and Publication II entail background knowledge on ordinary and stochastic differential equations, both of which are formally defined in Section 2.2. Afterwards, two rather practical topics are examined: numerical approximations to compute state solutions and gradient computation in differential equation systems. These practical aspects are often overlooked by researchers; however, they are crucial to understand how differential equation modeling differs from standard machine learning tasks such as regression or classification.

- The last section briefly covers parameter learning methods in machine learning in general. After an introduction to point estimation, we touch upon variational inference as it is employed in Publication III and Publication V. This subsection is followed by stochastic gradient Markov chain Monte Carlo techniques on which our Publication IV is built. We conclude by explaining gradient-descent based numerical optimization, including second-order techniques.

Since the above-mentioned topics have been studied for decades, the rest of this chapter focuses only on what is relevant to our publications. Please note that an in-depth overview of reinforcement learning $(\mathrm{RL})$ is presented in the respective chapter. 


\subsection{Function Approximation}

This section reviews two commonly used function approximators in machine learning: neural networks and Gaussian processes. Here, we introduce only the basic concepts and advances that have enabled our proposed models. A thorough investigation of neural networks can be found in Goodfellow et al. (2016) and please see Schmidhuber (2015) for a timeline of the advances in deep learning. We refer the readers to Rasmussen and Williams (2006) to find out more about Gaussian processes and Liu et al. (2020) for a summary of recent and more scalable approaches.

\subsubsection{Neural Networks}

A standard neural network (NN) consists of simple processing units called neurons, which are connected to each other in a structured way. The application at hand often dictates internal organization of neurons. Regardless of the architecture, the network parameters are typically inferred by backpropagation, a technique to compute the gradient of a loss function with respect to each parameter by chain rule (Linnainmaa, 1970; Rumelhart et al., 1986). Thanks to their auto-differentiation feature, modern machine learning frameworks such as PyTorch (Paszke et al., 2019) and TensorFlow (Abadi et al., 2016) require only the forward model to perform backpropagation. In the following, we describe specific types of NNs that are relevant to our publications.

\section{Multi-Layer Perceptron (MLP)}

Multi-layer perceptron, also known as feedforward neural network or fully connected layers, is a class of NNs comprised of an input layer, an output layer and at least one hidden layer (McCulloch and Pitts, 1943; Rosenblatt, 1961). Each layer $l$ transforms an input vector $\mathrm{x}_{l}$ into an output vector $\mathbf{x}_{l+1}$ by performing affine matrix operations and by applying a possibly nonlinear function to the output:

$$
\mathbf{x}_{l+1}=\mathbf{f}^{(l)}\left(\mathbf{x}_{l}\right) \doteq \sigma_{l}\left(\mathbf{W}_{l} \mathbf{x}_{l}+\mathbf{b}_{l}\right) .
$$

Here, $\mathbf{W}_{l}, \mathbf{b}_{l}$, and $\sigma_{l}$ denote the weight, bias, and activation at layer $l$. Note that often the collection $\left(\mathbf{W}_{l}, \mathbf{b}_{l}\right)$ is called "weights". Finally, a simple MLP performs a chain of operations $\mathbf{f}^{(n)}\left(\mathbf{f}^{(n-1)}\left(\ldots \mathbf{f}^{(0)}(\mathbf{x})\right)\right)$, where $\mathbf{x}$ is the original input.

\section{Convolutional Neural Network (CNN)}

Convolutional neural networks are another class of NNs designed particularly for extracting patterns in a hierarchical manner (LeCun et al., 1989). Inspired by the neurons in human visual system, CNN layers perform the "convolution operation". In the context of image processing, convolutions 
operate on $3 D$ input tensors, where two indices are reserved for the spatial coordinates and one index for the channel. Typically, each layer of a CNN stores $4 D$ kernel parameters $\mathbf{K}$, where $K_{i j m n}$ gives the connection strength between a unit in the $n$ 'th channel of the output and a unit in the $m$ 'th channel of the input, and $i$ and $j$ denotes the row and column offsets. Given a $3 D$ input $\mathbf{X}^{(l)}$, which could be the original input image or the output of the previous layer, each layer performs the following convolution operation:

$$
X_{p, r, n}^{(l+1)}=\sum_{i, j, m} X_{p+i, r+j, m}^{(l)} K_{i j m n} .
$$

Similar to MLPs, the convolutional layers are very conveniently stacked to extract features with an increasing complexity. Computer vision applications typically enrich the above operation by padding the input image and by shifting the filter by different amounts, called stride. Also, nonlinear activation functions applied at layer outputs and pooling layers to downsample feature maps are known to boost model performance. We refer the reader to Goodfellow et al. (2016) for more details and recent advances in CNNs.

\section{Bayesian Neural Network (BNN)}

Despite the universal function approximation guarantees, standard NNs suffer from a number of limitations. First, since deeper and wider NNs with more parameters almost always achieve better fits to the training data, blindly minimizing an error function with respect to model parameters prevents us from determining the appropriate level of complexity. To tackle this problem, researchers typically resort to ad-hoc regularization terms (Hanson and Pratt, 1988; Krogh and Hertz, 1992), terminate the optimization procedure prematurely (Prechelt, 1998) or use validation sets. Second, computing point estimates does not allow us to quantify the uncertainty on the unknown parameters, which makes risk assessment and decision making difficult in safety-critical applications such as autonomous driving.

Bayesian modeling provides an alternative and automated way of handling model uncertainty by penalizing complex models and by maintaining posterior beliefs on the parameters. The pioneering work by MacKay (1992) describes the first Bayesian treatment of neural networks by placing a Gaussian prior distribution on the weights. Later, Neal (1994) specifies different types of priors and also infers NN parameters by hybrid Monte Carlo (Duane et al., 1987; Neal et al., 2011). We revisit the parameter learning and Bayesian perspective in Subsection 2.3.1.

\subsubsection{Gaussian Processes}

Gaussian processes (GPs) are stochastic processes such that any finite set of its random variables follows a multivariate Gaussian distribution 
(Rasmussen and Williams, 2006). In machine learning, GPs are typically used to define priors on the functions, denoted by

$$
f(\mathbf{x}) \sim \mathcal{G P}\left(\mu(\mathbf{x}), k\left(\mathbf{x}, \mathbf{x}^{\prime}\right)\right)
$$

where $f: \mathbb{R}^{d} \rightarrow \mathbb{R}$ maps $d$-dimensional inputs into one-dimensional outputs (Please see, e.g., (Boyle and Frean, 2005; Williams et al., 2007) for the generalization of this framework to multi-output functions). A GP is fully specified in terms of its mean

$$
\mathbb{E}[f(\mathbf{x})]=\mu(\mathbf{x})
$$

and covariance function

$$
\operatorname{cov}\left[f(\mathbf{x}), f\left(\mathbf{x}^{\prime}\right)\right]=k\left(\mathbf{x}, \mathbf{x}^{\prime}\right)
$$

where $\mathbf{x}$ and $\mathrm{x}^{\prime}$ denote input points. In this dissertation, we abide by the very typical zero mean assumption: $\mu(\mathbf{x})=0$. The covariance between two function outputs is determined solely by the kernel function $k(\cdot, \cdot)$, which simply gives a measure of similarity between two inputs.

For any set of input points $X=\left[\mathbf{x}_{1}, \mathbf{x}_{2}, \ldots, \mathbf{x}_{N}\right]^{T} \in \mathbb{R}^{N \times d}$, a GP prior defines a multivariate Gaussian distribution over function outputs $F=$ $\left[f\left(\mathbf{x}_{1}\right), f\left(\mathbf{x}_{2}\right), \ldots, f\left(\mathbf{x}_{N}\right)\right]^{T} \in \mathbb{R}^{N}$ :

$$
\left[\begin{array}{c}
f\left(\mathbf{x}_{1}\right) \\
\vdots \\
f\left(\mathbf{x}_{N}\right)
\end{array}\right] \sim \mathcal{N}\left(\left[\begin{array}{c}
0 \\
\vdots \\
0
\end{array}\right],\left[\begin{array}{ccc}
k\left(\mathbf{x}_{1}, \mathbf{x}_{1}\right) & \cdots & k\left(\mathbf{x}_{1}, \mathbf{x}_{N}\right) \\
\vdots & \ddots & \vdots \\
k\left(\mathbf{x}_{N}, \mathbf{x}_{1}\right) & \cdots & k\left(\mathbf{x}_{N}, \mathbf{x}_{N}\right)
\end{array}\right]\right)
$$

which can be written more compactly as follows:

$$
p(F)=\mathcal{N}(F \mid \mathbf{0}, K) .
$$

Here, $K$ is the covariance matrix with entries $K_{i j}=k\left(\mathbf{x}_{i}, \mathbf{x}_{j}\right)$. After observing a collection of noise-free data points, usually in form of input/output pairs $(X, F)$, a GP specifies a Gaussian posterior predictive distribution at any test point $\mathrm{x}^{*}$ :

$$
\begin{aligned}
p\left(f\left(\mathbf{x}^{*}\right) \mid X, F\right)=\mathcal{N}\left(f\left(\mathbf{x}^{*}\right) \mid\right. & k\left(\mathbf{x}^{*}, X\right) k(X, X)^{-1} F, \\
& \left.k\left(\mathbf{x}^{*}, \mathbf{x}^{*}\right)-k\left(\mathbf{x}^{*}, X\right) k(X, X)^{-1} k\left(X, \mathbf{x}^{*}\right)\right) .
\end{aligned}
$$

Informally speaking, posterior mean would have similar values to the outputs of those states that are close to $\mathrm{x}^{*}$, and the variance grows as $\mathrm{x}^{*}$ goes away from the data points.

The computational bottleneck in GP inference is the matrix inversion in (2.1), which scales cubically $O\left(N^{3}\right)$ with the size of the input set. A widely used technique to enable GP models in big data regimes is the inducing variable approach (Seeger et al., 2003; Quiñonero-Candela and Rasmussen, 
2005; Titsias, 2009; Hensman et al., 2013). The idea is built on augmenting the GP with a set of inducing points $Z=\left[\mathbf{z}_{1}, \mathbf{z}_{2}, \ldots, \mathbf{z}_{M}\right]^{T}$ that live in the same space as $X$, and corresponding outputs $U=\left[u_{1}, u_{2}, \ldots, u_{M}\right]^{T}$ with $u_{m} \equiv f\left(\mathbf{x}_{m}\right)$. In turn, the predictive posterior distribution is computed conditioned on the inducing set:

$$
\begin{aligned}
p\left(f\left(\mathbf{x}^{*}\right) \mid Z, U\right)=\mathcal{N}\left(f\left(\mathbf{x}^{*}\right) \mid\right. & k\left(\mathbf{x}^{*}, Z\right) k(Z, Z)^{-1} U, \\
& \left.k\left(\mathbf{x}^{*}, \mathbf{x}^{*}\right)-k\left(\mathbf{x}^{*}, Z\right) k(Z, Z)^{-1} k\left(Z, \mathbf{x}^{*}\right)\right) .
\end{aligned}
$$

The resulting model is a low-rank inducing point approximation of GPs, also called sparse GP (SGP). With inducing approximation, computational cost of predictive posterior becomes $O\left(N M^{2}\right)$, which would give a significant improvement when $M \ll N$. Inducing points are typically learned by variational inference (Titsias, 2009; Hensman et al., 2013) in which inducing locations are treated as hyperparameters. Also, the original exact GP formulation can be retrieved by setting $Z=X$ and $U=F$.

\subsection{Differential Equations}

We now introduce ordinary and stochastic differential equations. Without loss of generality, this section treats time $t$ as the independent variable on which state solutions $\mathrm{x}(t)$ depends. After giving formal definitions, we illustrate the concepts on Van der Pol oscillator, a parametric differential equation system describing the motion of a non-conservative oscillator. The section is concluded by the numerical approximations to state solutions and gradient computation.

\subsubsection{Ordinary Differential Equations}

Ordinary differential equation (ODE) systems are defined as

$$
\dot{\mathbf{x}}(t)=\frac{d \mathbf{x}(t)}{d t}=\mathbf{f}(\mathbf{x}(t), \mathbf{a}(t), t),
$$

where $\mathbf{x}(t) \in \mathcal{X} \in \mathbb{R}^{d}$ is the state vector of a $d$-dimensional dynamical system at time $t, \mathbf{a}(t) \in \mathcal{A} \in \mathbb{R}^{m}$ is the external control signal and $\dot{\mathbf{x}}(t) \in$ $\dot{\mathcal{X}} \in \mathbb{R}^{d}$ is the first order time derivative of $\mathrm{x}(t)$. The vector-valued and continuous (time) differential function $\mathrm{f}: \mathcal{X} \times \mathcal{A} \times \mathbb{R}_{+} \rightarrow \dot{\mathcal{X}}$ describes the system's evolution over time with $\mathbb{R}_{+}$denoting non-negative real numbers (Tenenbaum, 1985). The control signals are typically bounded within a fixed range $a_{i}^{\min } \leq a_{i}(t) \leq a_{i}^{\max }$.

Computing ODE solutions An ODE solution $\mathbf{x}(t)$ at time $t \in \mathbb{R}_{+}$is a function given by

$$
\mathbf{x}(t)=\mathbf{x}_{0}+\int_{0}^{t} \mathbf{f}(\mathbf{x}(\tau), \mathbf{a}(\tau), \tau) d \tau
$$


where $\mathbf{x}_{0}$ denotes the initial value and $\tau \in \mathbb{R}_{+}$is an auxiliary time variable. The above equation implies $\dot{\mathbf{x}}(\tau)=\mathbf{f}(\mathbf{x}(\tau), \mathbf{a}(\tau), t) \forall \tau \in[0, t]$ and thus $\mathbf{x}(t)$ has a continuous derivative everywhere.

The celebrated Picard's existence and uniqueness theorem states that an initial value problem given by $\dot{x}(t)=f(x, t)$ and $x(0)=x_{0}$ has a unique solution $x(t)$ if $f(x, t)$ satisfies the Lipschitz condition:

$$
\left|f\left(x_{1}, t\right)-f\left(x_{2}, t\right)\right| \leq K\left|x_{1}-x_{2}\right|, \quad \forall\left(x_{1}, t\right),\left(x_{2}, t\right) .
$$

Despite the uniqueness guarantee, there is no general recipe to analytically compute the solution $x(t)$. We examine the numerical methods to compute ODE solutions in Section 2.2.3.

Higher dimensional ODEs Our discussion so far has concentrated on firstorder ODE systems. In general, the order of an ODE system is determined by the highest order time-derivative involved in the equation. For instance, a $p$ 'th order ODE is defined as

$$
\begin{aligned}
& \mathbf{x}^{(p)}(t)=F\left(\mathbf{x}(t), \mathbf{x}^{(1)}(t), \ldots, \mathbf{x}^{(p-1)}(t), \mathbf{a}(t), t\right) \\
& \mathbf{x}^{(r)}(t) \equiv \frac{d^{r} \mathbf{x}(t)}{d t^{r}} \in \mathbb{R}^{d}, \quad \forall r \in[1, \ldots, p],
\end{aligned}
$$

where $\mathbf{x}^{(r)}(t)$ stands for the $r$ 'th order derivative and $F$ is the $p$ 'th order time derivative function that takes as input all lower order derivatives. Note that any higher order ODE appears as a special case of a first-order system defined in an augmented space:

$$
\begin{aligned}
\mathbf{z} & \equiv\left[\mathbf{x}, \mathbf{x}^{(1)}, \ldots, \mathbf{x}^{(p-1)}\right] \in \mathbb{R}^{d p} \\
\mathbf{f}(\mathbf{z}(t), \mathbf{a}(t), t) & =\left[\mathbf{x}^{(1)}, \mathbf{x}^{(2)}, \ldots, \mathbf{x}^{(p-1)}, F(\mathbf{z}, \mathbf{a}, t)\right] \in \mathbb{R}^{d p},
\end{aligned}
$$

where [.] column stacks vectors and we drop $(t)$ from our state notation for clarity.

\subsubsection{Stochastic Differential Equations}

Stochastic differential equations (SDEs) are informally defined as noisedriven ODEs (Øksendal, 2014; Särkkä and Solin, 2019):

$$
\frac{d \mathbf{x}(t)}{d t}=\mathbf{f}(\mathbf{x}(t))+\boldsymbol{\sigma}(\mathbf{x}(t)) W_{t}
$$

We first note that without loss of generality, we drop any explicit dependence on time or control signals as they are not relevant to any of our publications. Above $\mathbf{f}\left(\mathbf{x}_{t}\right)$ and $\boldsymbol{\sigma}\left(\mathbf{x}_{t}\right)$ are referred to as drift and diffusion functions. $W_{t}$ denotes a multivariate continuous-time stochastic process, called Wiener process or white noise. The Wiener process always starts from a zero initial state $W_{0}=\mathbf{0}$, and the increments $W_{t+\Delta t}-W_{t} \sim \mathcal{N}(\mathbf{0}, \Delta t I)$ 

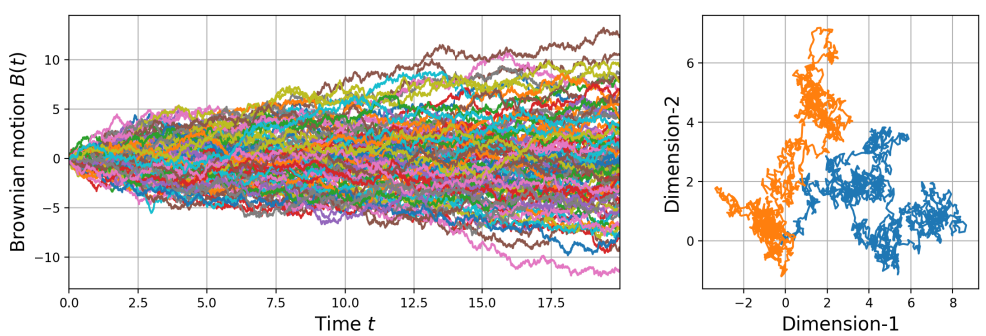

Figure 2.1. Two Brownian motion illustrations. The left panel demonstrates 100 different realizations of the one dimensional Brownian motion plotted over time. On the right are two examples of $2 D$ motions.

follow a Gaussian distribution with standard deviation $\sqrt{\Delta t}$, i.e., are also independent from the past values $W_{s}, s \leq t$. Due to historical connections, Wiener process is also called Brownian motion, which was first proposed to study the random motions of particles suspended in fluid. More formally, Brownian motion $B(t)$ is the solution to the following stochastic differential equation:

$$
\frac{d B_{t}}{d t}=W_{t}
$$

We illustrate one and two dimensional Brownian motions in Figure 2.1.

The theory of ODEs requires that the right hand side of a differential system must be continuous whereas the white noise process in (2.5) is discontinuous. Since the differential does not exist in the ordinary sense, we turn our attention to Itô calculus which allows us to compute integrals with respect to stochastic processes. By multiplying both sides of (2.5) by $d t$ and plugging in the definition of Brownian motion (2.6), we obtain the following differential:

$$
d \mathbf{x}(t)=\mathbf{f}(\mathbf{x}(t)) d t+\boldsymbol{\sigma}(\mathbf{x}(t)) d B_{t}
$$

Integrating both sides of above equation gives us the stochastic state solution:

$$
\mathbf{x}(t)=\mathbf{x}_{0}+\int_{0}^{t} \mathbf{f}(\mathbf{x}(\tau)) d \tau+\int_{0}^{t} \sigma(\mathbf{x}(\tau)) d B_{\tau},
$$

where the second integral is an Itô integral. We refer the readers to Øksendal (2014) and Särkkä and Solin (2019) for a more detailed derivation of SDE state solutions.

\section{Example: Van der Pol Oscillator}

As an example, we examine Van der Pol (VDP) oscillator, a parametric second-order time-invariant ODE system that evolves according to the following differential equation:

$$
\frac{d^{2} x}{d t^{2}}-\mu\left(1-x^{2}\right) \frac{d x}{d t}+x=0
$$


where we set the only parameter $\mu=1$. By defining $y \triangleq \frac{d x}{d t}$ and organizing the terms, we obtain the following two dimensional first-order system:

$$
\frac{d}{d t}\left[\begin{array}{l}
x \\
y
\end{array}\right]=\left[\begin{array}{c}
y \\
\left(1-x^{2}\right) y-x
\end{array}\right]
$$

To illustrate how Brownian motion impacts the deterministic ODE trajectory, we contrast ODE trajectories evolving according to VDP dynamics (2.8) with SDE trajectories evolving according to the following differential:

$$
d\left[\begin{array}{l}
x \\
y
\end{array}\right]=\left[\begin{array}{c}
y \\
\left(1-x^{2}\right) y-x
\end{array}\right] d t+\sigma\left(\left[\begin{array}{l}
x \\
y
\end{array}\right]\right) \cdot I d B_{t}
$$

where $I$ denotes the identity matrix. For notational convenience, we combine the concatenated states into a single variable: $\mathbf{x}=[x, y]^{T}$. We consider two constant diffusion functions $\sigma(\mathbf{x}, t)=0.05$ and $\sigma(\mathbf{x}, t)=0.25$, as well as a state-dependent diffusion $\sigma(\mathbf{x}, t)=3 \mathcal{N}\left(\mathbf{x} ;[-2,0]^{T}, I\right)$ with $\mathcal{N}$ denoting the Gaussian distribution. We integrate all systems with initial condition $\mathbf{x}_{0}=[1,0]^{T}$ for $t \in[0,30]$ (the details of integration are presented in Section 2.2.3). Figure 2.2 illustrates the trajectories in state space and also over time, please see the figure caption for more details.

\subsubsection{Numerical Solvers}

We now give a quick recap of numerical approximations to ODE and SDE state solutions. Note that this section places more emphasis on ODE approximations since they form the basis for their SDE counterparts.

\section{Numerical Approximations to ODEs}

The literature on numerical approximations to the solutions of ODEs dates back to 18 th century when Euler discovered the simplest numerical integration technique. A thorough overview of the numerical approximations is given in Butcher and Goodwin (2008) and Atkinson et al. (2011). In this subsection, we informally discuss numerical approximations relevant to our publications on a generic one-dimensional ODE system.

The simplest and least efficient numerical method is known as Euler's method. Relying on the standard derivative approximation, Euler's method computes the state solution $x\left(t_{n+1}\right)$ starting from the previous solution $x\left(t_{n}\right)$ as follows:

$$
x\left(t_{n+1}\right)=x\left(t_{n}\right)+h f\left(x_{n}, t_{n}\right), \quad h \equiv t_{n+1}-t_{n},
$$

where we use the shorthand notation $x_{n} \equiv x\left(t_{n}\right)$. Here, $h$ represents the step size and controls the local truncation error, i.e., the error made in a single step, which can be obtained via the Taylor approximation of the state solution (Butcher and Goodwin, 2008):

$$
x^{\text {true }}\left(t_{n+1}\right)-x\left(t_{n+1}\right)=\frac{1}{2} h^{2} \ddot{x}(t)+O\left(h^{3}\right) .
$$


(a1) VDP ODE Phase Portrait
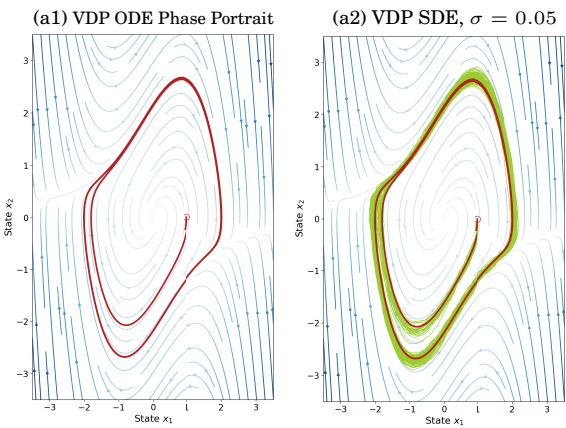

(b1) ODE state trajectories over time
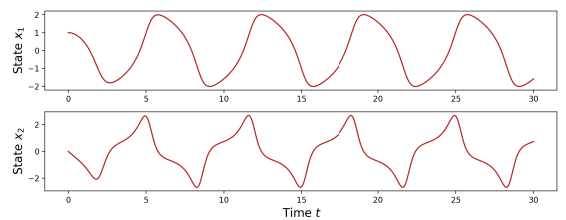

(b3) SDE state trajectories, $\sigma=0.25$
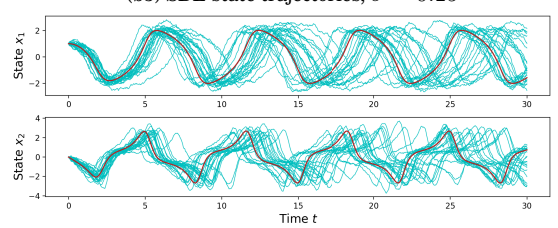

(a3) VDP SDE, $\sigma=0.25$
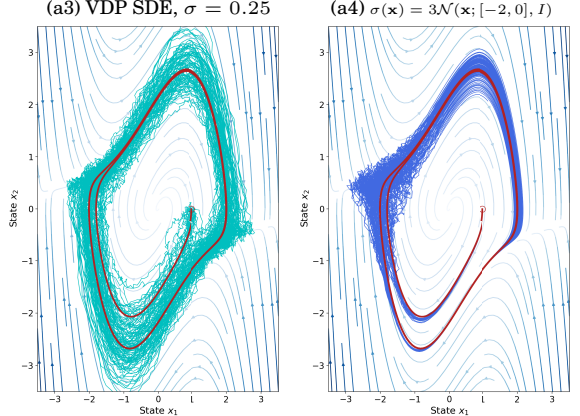

(b2) SDE state trajectories, $\sigma=0.05$
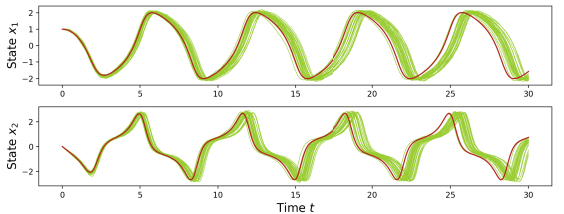

(b4) SDE state trajectories, $\sigma(\mathbf{x})=\mathcal{N}(\mathbf{x} ;[-2,0], I)$
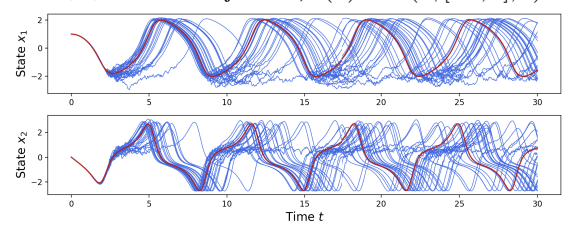

Figure 2.2. An illustration of Van der Pol oscillator. The ODE plots are generated using (2.8) while SDE trajectories follow (2.9). (a) Phase portraits in which each axis corresponds to one state. The empty circles and red curves show the initial value and ODE state trajectory, which are identical across all four figures. The streamplots on the background demonstrate the vector field, i.e., the time derivative (2.8). Streams get darker as the magnitude of the vector field grows. Colored non-smooth curves are 25 trajectory samples from each SDE system. (a2-a3) As expected, variance of the sampled trajectories grow with diffusion and the trajectories are corrupted everywhere due to constant diffusion. (a4) While the dynamics are highly chaotic near the diffusion hotspot $[-2,0]$, they become almost deterministic and even converge to the limit cycle far away. (b) The same state trajectories plotted over time. (b1) The system already reaches the limit cycle near $t=7$. (b2-b4) The phase difference between the ODE state solution and SDE state distribution $p\left(\mathbf{x}_{t}\right)$ grows as integration proceeds. 
The first implication of the above equation is that decreasing step size $h$ improves the quality of approximation, leading to the classical trade-off between speed and accuracy. More importantly, Euler's method becomes less accurate as the local gradient changes more abruptly, which is due to the first term on the right hand side (rhs) of the equality. Such equations are sometimes called stiff equations, although there is no consensus on the formal definition of stiffness (Butcher and Goodwin, 2008).

Stability of Euler's method can be improved by simply performing more computations in a single step. For instance, taking an additional step to compute an averaged slope leads to the so-called Heun's method (Heun, 1900):

$$
\begin{aligned}
& \tilde{x}\left(t_{n+1}\right)=x\left(t_{n}\right)+h f\left(x_{n}, t_{n}\right) \\
& x\left(t_{n+1}\right)=x\left(t_{n}\right)+\frac{h}{2}\left[f\left(x_{n}, t_{n}\right)+f\left(\tilde{x}_{n+1}, t_{n+1}\right)\right] .
\end{aligned}
$$

Being a second order technique, Heun's method is one of the simplest example of a class of numerical algorithms known as Runge-Kutta (RK) methods (Runge, 1895; Kutta, 1901). Due to their ease of implementation in digital computers, the literature on higher order RK solvers quickly advanced (Butcher and Goodwin, 2008). In our publications, we often utilize RK4 solver, a popular fourth-order procedure with $O\left(h^{5}\right)$ local truncation error given by the following set of equations:

$$
\begin{aligned}
z_{1} & =x_{n} \\
z_{2} & =x_{n}+\frac{h}{2} f\left(z_{1}, t_{n}\right) \\
z_{3} & =x_{n}+\frac{h}{2} f\left(z_{2}, t_{n}+\frac{h}{2}\right) \\
z_{4} & =x_{n}+h f\left(z_{3}, t_{n}+\frac{h}{2}\right) \\
x\left(t_{n+1}\right) & =x\left(t_{n}\right)+\frac{h}{6}\left[f\left(z_{1}, t_{n}\right)+2 f\left(z_{2}, t_{n}+\frac{h}{2}\right)+2 f\left(z_{3}, t_{n}+\frac{h}{2}\right)+f\left(z_{4}, t_{n}+h\right)\right] .
\end{aligned}
$$

A clever direction to further improve higher-order RK methods is to set the step size $h$ dynamically, leading to adaptive-step ODE solvers (also known as Fehlberg methods) (Fehlberg, 1969). These solvers simultaneously compute $x\left(t_{n+1}\right)-x\left(t_{n}\right)$ by using two methods of different orders, and then the higher-order formula is used to estimate the local approximation error of the lower-order formula. In turn, the error is used to tune the step size. Despite computing two formulae, such methods are highly compute friendly. For instance, one of the most commonly used adaptive-step solver, $R K 45$ (dopri5), uses only six function evaluations to compute forth and fifth order approximations to $x\left(t_{n+1}\right)-x\left(t_{n}\right)$ (Dormand and Prince, 1980).

\section{Numerical Approximations to SDEs}

Numerical approximations to SDE state solutions are typically built upon their ODE counterparts. A detailed categorization of numerical SDE 
approximations are presented in Särkkä and Solin (2019). Here, we explain the only solver involved in our publications, namely, Euler-Maruyama (EM) method.

Given a set of time points $\left[t_{0}, \ldots, t_{N}\right]$, we first divide the interval $\left[t_{0}, t_{N}\right]$ into $M \gg N$ steps $\left[\tilde{t}_{0}, \ldots, \tilde{t}_{M}\right]$ of fixed length $h=\left(t_{N}-t_{0}\right) / M$. We then sample the Wiener increments as $\Delta B_{m} \sim \mathcal{N}(\mathbf{0}, h \cdot I), \forall m \in[0, \ldots, M]$, and iteratively compute the following:

$$
x\left(\tilde{t}_{m+1}\right)=x\left(\tilde{t}_{m}\right)+f\left(x_{m}\right) h+\sigma\left(x_{m}\right) \Delta B_{m} .
$$

The strong order of convergence of EM is given as follows:

$$
\mathbb{E}\left[\left|x^{\text {true }}\left(\tilde{t}_{m+1}\right)-x\left(\tilde{t}_{m+1}\right)\right|\right] \leq K \sqrt{h}
$$

for a constant $K$. Therefore, similar to Euler's method, the accuracy of state solutions increase with $M$.

\subsubsection{Gradient Computation}

All our publications except IV require approximating unknown time differentials by black-box function approximators. In the following we describe different strategies to learn the parameters of an ODE system driven forward by $\mathrm{f}_{\boldsymbol{\theta}}$ with $\boldsymbol{\theta} \in \mathbb{R}^{P}$ denoting the parameters. Please see Publication II for a discussion on SDE parameter learning.

In the most general sense, the learning objectives of our interest can be described as minimizing the integral of an arbitrary loss $\ell(\mathbf{x}(t) ; \mathcal{D})$ that involves forward simulated states $\mathbf{x}(t)=\mathbf{x}_{0}+\int_{0}^{t} \mathbf{f}_{\boldsymbol{\theta}}\left(\mathbf{x}_{\tau}\right) d \tau$ and a dataset $\mathcal{D}$ :

$$
\min _{\boldsymbol{\theta}} L=\int_{0}^{T} \ell(\mathbf{x}(t) ; \mathcal{D}) d t,
$$

where we omit the control signals and the explicit dependence on time for clarity. Any optimization algorithm based on gradient descent would need to compute the gradient of forward simulated states with respect to model parameters: $\frac{d \mathbf{x}(t)}{d \boldsymbol{\theta}} \in \mathbb{R}^{d \times P}$. The majority of machine learning problems requires countably many tensor operations to compute the loss, allowing the gradients to be computed trivially by the chain rule. Nonetheless, forward simulated states are given by continuous-time integrals, which makes the gradient computation non-trivial. Next, we summarize three strategies to compute $\frac{d \mathbf{x}(t)}{d \boldsymbol{\theta}}$.

\section{Backpropogate through Numerical Solver}

The first and simplest approach is to store the entire computational graph built by the numerical integration, and backpropogate through it. Naturally, this technique yields the correct gradients given an accurate automatic differentiation framework. Nonetheless, a long backpropogation tree 
may easily cause vanishing or exploding gradients issues that are commonly observed in recurrent neural networks (Pascanu et al., 2013). Also, as pointed out in Zhuang et al. (2020), the backward computational graph would unnecessarily include the intermediate calculations performed during the step size search in case of an adaptive solver.

\section{Sensitivity Equations}

The goal of sensitivity analysis is to understand how the model responses change with variations in the input variables (Confalonieri et al., 2010; Weber et al., 2018). The local sensitivity of ODE models was introduced in a seminal work by Petar and Heller (1967) and recently revisited in the context of mechanistic modeling of biochemical reaction networks (Fröhlich et al., 2017). The analysis starts with considering the time derivative of the target gradient denoted by $S(t)$ below:

$$
\frac{d}{d t} \underbrace{\frac{d \mathbf{x}(t, \boldsymbol{\theta})}{d \boldsymbol{\theta}}}_{S(t)}=\frac{d}{d \boldsymbol{\theta}} \frac{d \mathbf{x}(t, \boldsymbol{\theta})}{d t}=\frac{d}{d \boldsymbol{\theta}} \mathbf{f}_{\boldsymbol{\theta}}(\mathbf{x}(t, \boldsymbol{\theta})) \in \mathbb{R}^{d \times P},
$$

where we denote a state by $\mathbf{x}(t, \boldsymbol{\theta})$ to explicitly show its dependence on the parameters $\boldsymbol{\theta}$. Since both inputs to $\mathrm{f}_{\boldsymbol{\theta}}$, namely $\boldsymbol{\theta}$ and $\mathbf{x}(t, \boldsymbol{\theta})$, are functions of $\theta$, the rhs term can be computed by total derivation:

$$
\overbrace{\frac{d}{d t} \frac{d \mathbf{x}(t, \boldsymbol{\theta})}{d \boldsymbol{\theta}}}^{\dot{S}(t)}=\overbrace{\frac{\partial \mathbf{f}_{\boldsymbol{\theta}}(\mathbf{x}(t, \boldsymbol{\theta}))}{\partial \mathbf{x}}}^{J(t)} \overbrace{\frac{d \mathbf{x}(t, \boldsymbol{\theta})}{d \boldsymbol{\theta}}}^{S(t)}+\overbrace{\frac{\partial \mathbf{f}_{\boldsymbol{\theta}}(\mathbf{x}(t, \boldsymbol{\theta}))}{\partial \boldsymbol{\theta}}}^{R(t)} .
$$

The terms $R(t) \in \mathbb{R}^{d \times P}$ and $J(t) \in \mathbb{R}^{d \times d}$ can easily be obtained by auto differentiating the output of $\mathbf{f}_{\boldsymbol{\theta}}$ with respect to its inputs. The target gradient is then given by the following ODE, hence also called forward sensitivities:

$$
S(t)=S_{0}+\int_{0}^{t} J(\tau) S(\tau)+R(\tau) d \tau
$$

If the initial state $\mathbf{x}_{0}$ is known, $\mathbf{S}_{0} \equiv \frac{d \mathbf{x}_{0}}{d \boldsymbol{\theta}}$ becomes a zero-matrix. Alternatively, one could define $\mathrm{x}_{0}$ as a free parameter and the sensitivity equations would also give the gradient $\frac{d L}{d \mathbf{x}_{0}}$.

The state solutions (2.4) and the sensitivity ODE (2.13) can be computed together in a single call to numerical ODE solver, which leads to an $d(1+P)$ dimensional ODE system. If the loss $J$ is computed over a sequence of length $T$, the memory footprint becomes $O(T d P)$. Therefore, this technique is not suitable for computing the gradients of overparameterized functions such as neural networks.

\section{Adjoint Method}

The adjoint equation provides a computationally efficient alternative to forward sensitivities. Even though the method antedate the first works 
on optimal control (Pontryagin et al., 1962; Pontryagin, 2018), the neural ODE (NODE) breakthrough (Chen et al., 2018b) introduced the method to the machine learning community. The method starts by considering the gradient $\nabla_{\boldsymbol{\theta}} \mathcal{L}$ of a Lagrangian involving both the loss function and ODE constraint:

$$
\mathcal{L}=\int_{0}^{T} \ell(\mathbf{x}(t) ; \mathcal{D})+\boldsymbol{\lambda}(t)^{T}\left(\dot{\mathbf{x}}(t)-\mathbf{f}_{\boldsymbol{\theta}}(\mathbf{x}(t))\right) d t,
$$

where $\boldsymbol{\lambda}(t)$ denotes the Lagrange multiplier, or costate, at time $t$. After a series of algebraic manipulations, we obtain the dynamics of the multipliers (Pontryagin, 2018; Bradley, 2013):

$$
\dot{\boldsymbol{\lambda}}(t)=-\boldsymbol{\lambda}(t)^{T} \frac{\partial \mathbf{f}_{\boldsymbol{\theta}}(\mathbf{x}(t))}{\partial \mathbf{x}(t)}+\frac{\partial \ell(\mathbf{x}(t) ; \mathcal{D})}{\partial \mathbf{x}(t)}
$$

where $\boldsymbol{\lambda}(T)=\mathbf{0}$ and the integral is computed in the backward direction. Our target gradient also follows an integral:

$$
\frac{d L}{d \boldsymbol{\theta}}=\int_{0}^{T}-\boldsymbol{\lambda}(t)^{T} \frac{\partial \mathbf{f}_{\boldsymbol{\theta}}(\mathbf{x}(t))}{\partial \boldsymbol{\theta}}+\frac{\partial \ell(\mathbf{x}(t) ; \mathcal{D})}{\partial \boldsymbol{\theta}} d t
$$

Similar to the forward sensitivities, the costates (2.14) and the loss integral (2.15) are executed concurrently. Since the gradient of the loss is computed in the backward direction, the adjoints are viewed as the continuous-time analog of backpropogation. Also, the memory consumption of the loss integral (2.15) is independent of $T$, hence improving the resource usage in orders of magnitude.

In their recent presentation of the adjoint method, Chen et al. (2018b) propose to discard the forward states $\mathrm{x}(t)$ given by (2.4), and re-compute them during backpropagation (2.15) for memory considerations. In a more recent work, it was demonstrated that the forward and backward state trajectories are not identical (Zhuang et al., 2020), which leads to numerical inaccuracies. The authors resolve this problem by their adaptive checkpoint algorithm (ACA) that records the forward trajectory while also controlling memory cost. We utilized ACA in our Publication V, and experimentally verify its superiority over the standard adjoint method.

\subsection{A Primer on Parameter Inference}

We now examine parameter inference in machine learning models. Given a dataset $\mathcal{D}=\left\{\mathbf{x}_{i}\right\}_{i=1}^{N}$ consisting of $N$ data points, the maximum likelihood (ML) approach aims to find a set of parameters $\boldsymbol{\theta} \in \mathbb{R}^{P}$ that maximizes the likelihood (Bishop, 2006):

$$
\max _{\boldsymbol{\theta}} p(\mathcal{D} \mid \boldsymbol{\theta})
$$


The data points are typically assumed to be independently and identically distributed (iid), which allows the likelihood to be factorized. We can further simplify the likelihood by maximizing its logarithm instead:

$$
\max _{\boldsymbol{\theta}} \sum_{i=1}^{N} \log p\left(\mathbf{x}_{i} \mid \boldsymbol{\theta}\right) .
$$

Above optimization problem (2.17) has the same optima as the original formulation (2.16) since $\log (\cdot)$ is a monotonic function. This ML approach leads to a so-called point estimate for $\theta$, which does not provide any measure of uncertainty. Nonetheless, many applications require to know how much we can trust a model in its predictions. Bayes' rule provides a natural framework to express our degree of belief in form of probability distributions. Given a prior distribution $p(\boldsymbol{\theta})$ on the unknown quantities (parameters $\boldsymbol{\theta}$ here), Bayes' theorem gives the formula for the posterior distribution, which reflects our belief after seeing data:

$$
p(\boldsymbol{\theta} \mid \mathcal{D})=\frac{p(\mathcal{D} \mid \boldsymbol{\theta}) p(\boldsymbol{\theta})}{p(\mathcal{D})},
$$

where the denominator is independent of $\boldsymbol{\theta}$. When the likelihood function is complex or the prior is not conjugate to likelihood, deriving a closed form expression for the posterior distribution $p(\boldsymbol{\theta} \mid \mathcal{D})$ becomes too difficult. For such cases, we could directly maximize the posterior distribution (or its logarithm), a technique called maximum a posteriori (MAP) estimation:

$$
\max _{\boldsymbol{\theta}} \log p(\boldsymbol{\theta} \mid \mathcal{D})=\log p(\boldsymbol{\theta})+\sum_{i=1}^{N} \log p\left(\mathbf{x}_{i} \mid \boldsymbol{\theta}\right)+c,
$$

where the constant $c=-\log p(\mathcal{D})$ appears due to the denominator in (2.17). The only difference between ML (2.17) and MAP (2.18) estimates is the prior term, which acts as a regularizer (Bishop, 2006). Despite the regularization effect, MAP estimation still prevents us from maintaining a measure of uncertainty over the unknown parameters $\boldsymbol{\theta}$. Next, we discuss two alternative inference schemes that approximate intractable posteriors. This section is concluded by a brief introduction to numerical optimization.

\subsubsection{Variational Inference}

Variational inference (VI) aims to approximate an intractable posterior $p(\boldsymbol{\theta} \mid \mathcal{D})$ with a family of tractable densities $\mathscr{D}$ (Jordan et al., 1999; Blei et al., 2016). Once $\mathscr{D}$ is specified, we try to find the member $q(\boldsymbol{\theta})$ of the family closest to the true posterior

$$
\underset{q(\boldsymbol{\theta}) \in \mathscr{D}}{\arg \min } \operatorname{KL}[q(\boldsymbol{\theta}) \| p(\boldsymbol{\theta} \mid \mathcal{D})]
$$


where KL denotes the KL divergence that measures the discrepancy between two distributions. The density $q(\boldsymbol{\theta})$ is often referred to as variational posterior. Expanding the KL divergence (2.19) gives

$$
\mathrm{KL}[q(\boldsymbol{\theta}) \| p(\boldsymbol{\theta} \mid \mathcal{D})]=\mathbb{E}[\log q(\boldsymbol{\theta})]-\mathbb{E}[\log p(\mathcal{D}, \boldsymbol{\theta})]+\log p(\mathcal{D}),
$$

where the expectations are with respect to $q(\boldsymbol{\theta})$. This objective is again impossible to compute due to the unknown marginal $\log \operatorname{likelihood} \log p(\mathcal{D})$. However, since $\log p(\mathcal{D})$ does not depend on the variational posterior, we simply discard it and maximize (the minus of) the remaining terms, leading to evidence lower bound (ELBO):

$$
\begin{aligned}
\operatorname{ELBO}(q) & =\mathbb{E}[\log p(\mathcal{D}, \boldsymbol{\theta})]-\mathbb{E}[\log q(\boldsymbol{\theta})] \\
& =\mathbb{E}[\log p(\mathcal{D} \mid \boldsymbol{\theta})]-\mathrm{KL}[q(\boldsymbol{\theta}) \| p(\boldsymbol{\theta})]
\end{aligned}
$$

The first term above denotes the expected log likelihood, which favors distributions $q(\boldsymbol{\theta})$ that better fits the data. The second term computes the $\mathrm{KL}$ divergence between the approximate posterior and a user-specified prior distribution, which is typically chosen to inject our a priori knowledge about $\boldsymbol{\theta}$. Next we introduce two families of tractable approximate densities $\mathscr{D}$.

\section{Mean-Field Variational Inference}

The simplest class of approximate densities assumes that variational factors $\left\{\theta_{p}\right\}_{p=1}^{P}$ are independent of each other, which gives the following factorization:

$$
q(\boldsymbol{\theta})=\prod_{p=1}^{P} q_{p}\left(\theta_{p} ; \boldsymbol{\alpha}_{p}\right)
$$

with $q_{p}(\cdot)$ and $\boldsymbol{\alpha}_{p}$ denoting a factor-specific distribution and corresponding variational parameters. As an example application of mean-field VI, we consider Bayesian neural networks, which typically lead to analytically intractable posterior densities (Graves, 2011). A mean-field VI approximation places a Gaussian distribution on each individual weight and bias parameter:

$$
q_{p}\left(\theta_{p} ; \boldsymbol{\alpha}_{p}\right)=\mathcal{N}\left(\theta_{p} ; \mu_{p}, \sigma_{p}^{2}\right)
$$

Typically, the prior distribution $p(\boldsymbol{\theta})$ is chosen to be an isotropic Gaussian, allowing a closed form expression for the KL term in ELBO (2.20). Also, the expected log likelihood term can be approximated by Monte Carlo sampling:

$$
\mathbb{E}[\log p(\mathcal{D} \mid \boldsymbol{\theta})] \approx \frac{1}{L} \sum_{\ell=1}^{L} \log p\left(\mathcal{D} \mid \boldsymbol{\theta}^{\ell}\right), \quad \boldsymbol{\theta}^{\ell} \sim \mathcal{N}\left(\boldsymbol{\theta} ; \boldsymbol{\mu}, \boldsymbol{\sigma}^{2}\right)
$$

with $\boldsymbol{\mu}=\left[\mu_{1}, \ldots, \mu_{P}\right]^{T} \in \mathbb{R}^{P}$ and $\boldsymbol{\sigma}^{2}=\operatorname{diag}\left(\left[\sigma_{1}^{2}, \ldots, \sigma_{P}^{2}\right]\right) \in \mathbb{R}^{P \times P}$ denoting the collection of variational mean and variance parameters, noting that $\operatorname{diag}(\cdot)$ forms a diagonal matrix from its argument. 


\section{Amortized Variational Inference}

Mean-field VI has so-called local parameters for each unknown, which does not constitute a problem when the unknowns remain the same during training and testing. However, probabilistic models often associate data points with latent variables, implying the need for mechanisms to infer latent variables for (unseen) test points. Also, the number of variables increase with the dataset size, which makes large-scale applications difficult. To that end, amortized VI replaces free local parameters with a flexible and powerful global approximation, e.g., neural network. Below we consider one of the most popular models utilizing amortized inference, namely, variational auto-encoders (VAEs) (Kingma and Welling, 2014; Rezende et al., 2014).

The VAE framework assumes a data point $\mathrm{x}$ is generated by an unknown process that involves an unobserved continuous random variable $\mathrm{z}$. The unknown generative process is approximated by a non-linear decoder neural network $p_{\gamma}(\mathbf{x} \mid \mathbf{z})$ parameterized by $\gamma$, which makes the posterior distribution $p(\mathbf{z} \mid \mathbf{x})$ intractable. As an approximation to the posterior, a recognition model $q_{\psi}(\mathbf{z} \mid \mathbf{x})$ is proposed, which leads to the following expression for ELBO:

$$
\operatorname{ELBO}(\mathbf{x} ; \psi, \gamma)=\mathbb{E}_{q_{\psi}(\mathbf{z} \mid \mathbf{x})}\left[\log p_{\gamma}(\mathbf{x} \mid \mathbf{z})\right]-\operatorname{KL}\left[q_{\psi}(\mathbf{z} \mid \mathbf{x}) \| p(\mathbf{z})\right] .
$$

In VAEs, the recognition model is chosen to be a neural network $\mathbf{g}_{\psi}$ that outputs the parameters of the variational posterior distribution. This neural network is also known as encoder or inference network. The approximate posterior $q_{\psi}(\mathbf{z} \mid \mathbf{x})$ is typically chosen to allow reparameterization $\mathbf{z} \sim \mathbf{g}_{\psi}(\boldsymbol{\epsilon}, \mathbf{x})$ using an auxiliary noise variable $\boldsymbol{\epsilon}$, which in turns leads to the convenient Monte Carlo integration shown below:

$$
\begin{gathered}
\mathbf{z}^{(l)}=\mathbf{g}_{\psi}\left(\boldsymbol{\epsilon}^{(l)}, \mathbf{x}\right), \quad \boldsymbol{\epsilon}^{(l)} \sim p(\boldsymbol{\epsilon}) \\
\mathbb{E}_{q_{\psi}(\mathbf{z} \mid \mathbf{x})}\left[\log p_{\gamma}(\mathbf{x} \mid \mathbf{z})\right]=\mathbb{E}_{p(\boldsymbol{\epsilon})}\left[\log p_{\gamma}\left(\mathbf{x} \mid \mathbf{g}_{\psi}(\boldsymbol{\epsilon}, \mathbf{x})\right)\right] \approx \frac{1}{L} \sum_{l=1}^{L} \log p_{\gamma}\left(\mathbf{x} \mid \mathbf{g}_{\psi}\left(\boldsymbol{\epsilon}^{(l)}, \mathbf{x}\right)\right) .
\end{gathered}
$$

The original VAE paper proposes to set the approximate posterior to a multivariate Gaussian distribution with a diagonal covariance

$$
\log q_{\psi}(\mathbf{z} \mid \mathbf{x})=\log \mathcal{N}(\mathbf{z} ; \boldsymbol{\mu}, \boldsymbol{\sigma} I)
$$

and prior to a centered isotropic multivariate Gaussian:

$$
p(\mathbf{z})=\mathcal{N}(\mathbf{z} ; \mathbf{0}, I) .
$$

Putting all pieces together, we obtain the following approximation to the 
ELBO:

$$
\begin{aligned}
\operatorname{ELBO}(\mathbf{x} ; \psi, \gamma) & \approx \frac{1}{L} \sum_{\ell=1}^{L} \log p_{\gamma}\left(\mathbf{x} \mid \mathbf{z}^{(\ell)}\right)-\operatorname{KL}[\mathcal{N}(\boldsymbol{\mu}, \boldsymbol{\sigma} I) \| \mathcal{N}(\mathbf{0}, I)] \\
\mathbf{z}^{(\ell)} & =\mathbf{g}_{\psi}\left(\boldsymbol{\epsilon}^{(l)}, \mathbf{x}\right)=\boldsymbol{\mu}+\boldsymbol{\epsilon}^{(\ell)} \boldsymbol{\sigma} I \\
\boldsymbol{\epsilon}^{(\ell)} & \sim \mathcal{N}(\mathbf{0}, I) .
\end{aligned}
$$

The bound is maximized with respect to the global parameters $\psi$ and $\gamma$. The gradients are computed by reparameterization trick (Kingma and Welling, 2014), which is also known as stochastic backpropagation (Rezende et al., 2014).

\subsubsection{Stochastic Gradient Markov Chain Monte Carlo}

In Markov chain Monte Carlo (MCMC), the goal is to construct a Markov chain whose stationary distribution is the target density we wish to sample from. The stationary distribution is fully specified by the transition kernel, which describes the evolution of the Markov chain in probabilistic terms. Thus, the main research question in MCMC is how to construct the kernel given a target density that can be evaluated up to a normalizing constant. In this subsection, we focus on stochastic gradient MCMC (SGMCMC) methods, which utilize continuous stochastic dynamics to define transition kernels. For a rigorous treatment of transition kernels and a comprehensive survey of MCMC methods, please see (Andrieu et al., 2003; Bishop, 2006; Cemgil, 2014).

We start with the assumption that the target posterior density of our interest can be written as

$$
p(\boldsymbol{\theta} \mid \mathcal{D}) \propto \exp (-U(\boldsymbol{\theta}))
$$

where $U(\boldsymbol{\theta})$ is known as a potential energy function. In complicated models, often times direct sampling from $p(\boldsymbol{\theta} \mid \mathcal{D})$ is difficult while evaluating $U(\boldsymbol{\theta})$ for any input $\theta$ is easy. Ma et al. (2015) showed that an SDE of the form

$$
d \boldsymbol{\theta}=\mathbf{f}(\boldsymbol{\theta}) d t+\sqrt{2 D(\boldsymbol{\theta})} d W(t)
$$

with a special drift function that involves the gradient of $U(\boldsymbol{\theta})$

$$
\mathbf{f}(\boldsymbol{\theta})=-[D(\boldsymbol{\theta})+Q(\boldsymbol{\theta})] \nabla U(\boldsymbol{\theta})+\Gamma(\boldsymbol{\theta}), \quad \Gamma_{i}(\boldsymbol{\theta})=\sum_{j=1}^{P} \frac{\partial}{\partial \theta_{j}}\left[D_{i j}(\boldsymbol{\theta})+Q_{i j}(\boldsymbol{\theta})\right]
$$

has a stationary distribution proportional to our target density $p(\boldsymbol{\theta} \mid \mathcal{D})$. Above, $Q(\boldsymbol{\theta})$ and $D(\boldsymbol{\theta})$ are respectively skew-symmetric and positive semidefinite matrices with $(\cdot)_{i j}$ denoting the $i$ 'th row and $j$ 'th column. Typically, 
$Q(\boldsymbol{\theta})$ and $D(\boldsymbol{\theta})$ are chosen to reflect our choices in the traversing effects and the strength of the diffusion process. The rest of this subsection examines two special cases of this general framework for sampling from intractable posterior distributions.

\section{Stochastic Gradient Langevin Dynamics (SGLD)}

Since we are free to choose the matrices $D(\cdot)$ and $Q(\cdot)$, we simplify the above framework by setting $D(\boldsymbol{\theta})$ to the identity matrix and $Q(\boldsymbol{\theta})$ to a zeromatrix, which causes $\Gamma(\theta)$ to disappear as a by-product. In turn, EulerMaruyama discretization of the dynamics (2.21) gives a straightforward update equation representing the transition kernel:

$$
\begin{aligned}
\boldsymbol{\theta}_{t+1} & =\boldsymbol{\theta}_{t}+\epsilon_{t} \nabla U(\boldsymbol{\theta})+\boldsymbol{\eta}_{t} \\
\boldsymbol{\eta}_{t} & \sim \mathcal{N}\left(0,2 \epsilon_{t} \cdot I\right) .
\end{aligned}
$$

The above equation is identical to gradient ascent update up to the last noise term. As shown in Publication IV, a tempered version of (2.23) connects SGMCMC approaches with optimization. Furthermore, Welling and Teh (2011) showed that the gradient term $\nabla U(\boldsymbol{\theta})$ can be replaced by its unbiased estimate computed on mini-batches of data, which significantly improves the overall execution time.

\section{Hamiltonian Monte Carlo}

Hamiltonian (hybrid) Monte Carlo has been proven one of the most prominent MCMC methods that can efficiently explore posterior distributions (Duane et al., 1987; Neal et al., 2011; Ma et al., 2015). As suggested by its name, HMC simulates approximate Hamiltonian dynamics described by so-called generalized position $\boldsymbol{\theta}$ and momentum $\boldsymbol{\rho}$ variables. In turn, the Hamiltonian function gives the total energy of a closed system:

$$
\mathcal{H}(\boldsymbol{\theta}, \boldsymbol{\rho})=U(\boldsymbol{\theta})+g(\boldsymbol{\rho}) .
$$

where $U(\boldsymbol{\theta})$ was already defined as the potential energy. Analogously, $g(\boldsymbol{\rho})$ is known as the kinetic energy function and usually set as $g(\boldsymbol{\rho})=\frac{1}{2} \boldsymbol{\rho}^{T} M^{-1} \boldsymbol{\rho}$ with $M$ being a mass matrix. The time evolution of this system is expressed by Hamilton's equations:

$$
\begin{aligned}
& \frac{d \boldsymbol{\theta}}{d t}=\frac{\partial \mathcal{H}}{\partial \boldsymbol{\rho}}=M^{-1} \boldsymbol{\rho} \\
& \frac{d \boldsymbol{\rho}}{d t}=-\frac{\partial \mathcal{H}}{\partial \boldsymbol{\theta}}=-\nabla U(\boldsymbol{\theta})
\end{aligned}
$$

which is typically approximated by a symplectic solver such as the leapfrog integrator (Neal et al., 2011). HMC can be seen as a special case of the SGMCMC framework defined in the extended space $(\boldsymbol{\theta}, \boldsymbol{\rho})$. More specifically, setting $p(\boldsymbol{\theta}, \boldsymbol{\rho} \mid \mathcal{D}) \propto \exp (g(\boldsymbol{\rho})-U(\boldsymbol{\theta})), D(\boldsymbol{\theta}, \boldsymbol{\rho})=\mathbf{0}$, and $Q(\boldsymbol{\theta}, \boldsymbol{\rho})=$ $\left(\begin{array}{cc}0 & -I \\ I & 0\end{array}\right)$ leads to the dynamics equations given above. 


\subsubsection{Numerical Optimization}

This subsection introduces several concepts and ideas for solving unconstrained optimization problems of the following sort:

$$
\min _{\boldsymbol{\theta}} f(\boldsymbol{\theta})
$$

where $f: \mathbb{R}^{P} \rightarrow \mathbb{R}$ is an infinitely differentiable objective function to be optimized with respect to $\theta \in \mathbb{R}^{P}$. Descent methods that we examine here produce a sequence of iterates $\boldsymbol{\theta}_{k}$ to solve (2.24) (Boyd and Vandenberghe, 2004):

$$
\boldsymbol{\theta}_{k+1}=\boldsymbol{\theta}_{k}-\alpha_{k} \Delta \boldsymbol{\theta}_{k}
$$

with the step size $\alpha_{k}>0$ such that $f\left(\boldsymbol{\theta}_{k+1}\right)<f\left(\boldsymbol{\theta}_{k}\right)$. To choose the update direction $\Delta \boldsymbol{\theta}$, we consider the Taylor approximation of $f$ around $\boldsymbol{\theta}_{k}$ :

$$
f\left(\boldsymbol{\theta}_{k}-\Delta \boldsymbol{\theta}\right)=f\left(\boldsymbol{\theta}_{k}\right)-\nabla f_{\boldsymbol{\theta}_{k}} \Delta \boldsymbol{\theta}+\nabla^{2} f_{\boldsymbol{\theta}_{k}} \frac{(\Delta \boldsymbol{\theta})^{2}}{2 !}-\cdots+\nabla^{m} f_{\boldsymbol{\theta}_{k}} \frac{(\Delta \boldsymbol{\theta})^{m}}{m !}-\cdots
$$

with $\nabla^{m} f_{\boldsymbol{\theta}_{k}}=\frac{d^{m} f\left(\boldsymbol{\theta}_{k}\right)}{d \boldsymbol{\theta}^{m}}$ denotes the $m$ 'th order derivative at $\boldsymbol{\theta}_{k}$. If we consider only the first-order Taylor expansion, the maximum leverage out of moving along $\Delta \boldsymbol{\theta}$ can be obtained by choosing it to be in the same direction as the gradient:

$$
\boldsymbol{\theta}_{k+1}=\boldsymbol{\theta}_{k}-\alpha_{k} \nabla f_{\boldsymbol{\theta}_{k}}
$$

This update technique is thus called gradient descent. Since the Taylor approximation holds in a small neighborhood of $\boldsymbol{\theta}_{k}$, we choose step size $\alpha_{k}$ to be small in magnitude. Typically, too small step sizes slow down the convergence whereas setting them too big may cause diverging from the local optima (Nocedal and Wright, 2006).

Higher order Taylor expansions would naturally give better approximations to $f\left(\boldsymbol{\theta}_{k}-\Delta \boldsymbol{\theta}\right)$. We can maximize the second-order approximation

$$
f\left(\boldsymbol{\theta}_{k}+\Delta \boldsymbol{\theta}\right) \approx f\left(\boldsymbol{\theta}_{k}\right)-\nabla f_{\boldsymbol{\theta}_{k}} \Delta \boldsymbol{\theta}+\nabla^{2} f_{\boldsymbol{\theta}_{k}} \frac{(\Delta \boldsymbol{\theta})^{2}}{2}
$$

by setting the derivative to zero:

$$
\begin{aligned}
\frac{d}{d \Delta \boldsymbol{\theta}} f\left(\boldsymbol{\theta}_{k}+\Delta \boldsymbol{\theta}\right) & =-\nabla f_{\boldsymbol{\theta}_{k}}+\nabla^{2} f_{\boldsymbol{\theta}_{k}} \Delta \boldsymbol{\theta}=0 \\
\Delta \boldsymbol{\theta} & =\left(\nabla^{2} f_{\boldsymbol{\theta}_{k}}\right)^{-1} \nabla f_{\boldsymbol{\theta}_{k}} .
\end{aligned}
$$

This method is known as Newton's method since it depends on the iterative root finding technique developed by Newton and Raphson (Nocedal and Wright, 2006). Newton's method is more favorable to gradient descent thanks to its higher rate of converge. Nonetheless, in high dimensions, even storing the $P \times P$ Hessian matrix $\nabla^{2} f_{\boldsymbol{\theta}_{k}}$ becomes very expensive. For 
(a) Gradient Descent (10 iterations)

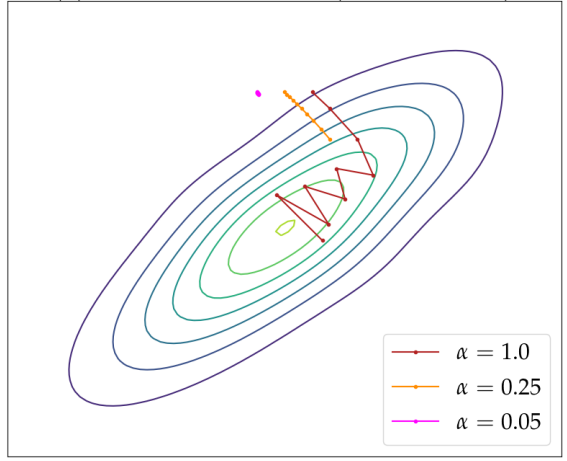

(c) LBFGS (10 iterations)

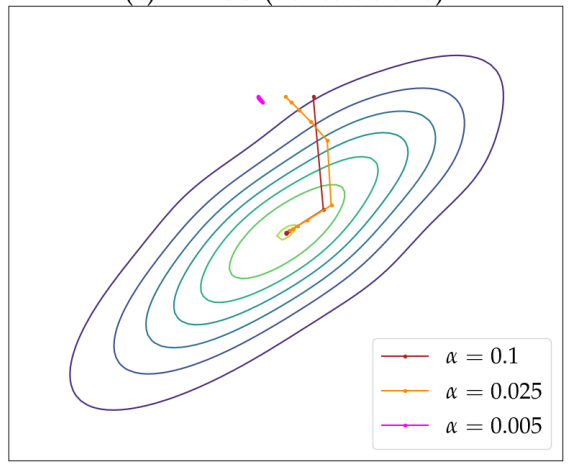

(b) Gradient Descent (100 iterations)

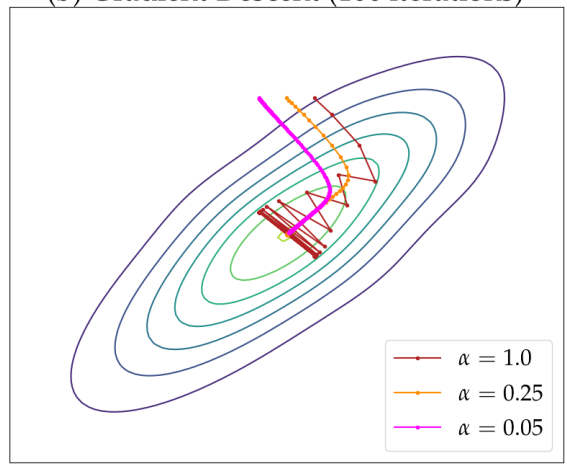

(d) LBFGS (100 iterations)

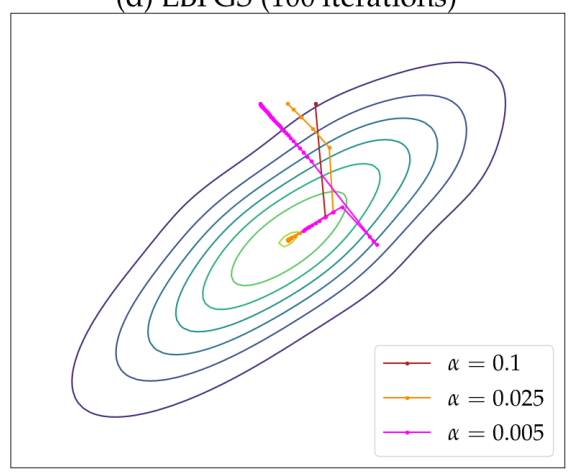

Figure 2.3. An illustration of gradient descent and L-BFGS methods on a convex problem. Each row shows the first 10 and 100 iterations of the same optimization procedure. The contours and curves show the optimization surface and iterations $\boldsymbol{\theta}_{1: T}$ with different fixed step sizes $\alpha$. (a-b) Gradient descent with high step sizes (red) can quickly reach near the minimum but oscillates around it instead of converging. Small step sizes require more iterations but optimization finally converges. (c) Typical L-BFGS step sizes are much smaller compared to gradient descent and only few iterations are sufficient for convergence due to the convexity of the problem. (d) Fixed step sizes may cause strange jumps (pink curve); therefore, it is recommended to choose $\alpha$ with line search (Nocedal and Wright, 2006).

such cases, Quasi-Newton methods provide an attractive alternative as they achieve similar convergence rates without computing the Hessian (Nocedal and Wright, 2006).

One widely used Hessian approximation is BFGS (due to its inventors Broyden, Fletcher, Goldfarb, and Shanno). The method iteratively updates an approximation to Hessian using the gradients $\nabla f_{\boldsymbol{\theta}_{k}}$ that are computed as optimization proceeds. A computationally more efficient BFGS variant, named limited memory BFGS (L-BFGS), uses only the most recent $L$ iterations to build an approximation to Hessian. Also, L-BFGS computes the matrix-gradient product in (2.26) using the two-loop recursion, which has $O(L P)$ time and space complexity (Nocedal and Wright, 2006). We contrast the gradient descent and L-BFGS on a convex optimization problem in Figure 2.3. 


\section{Gaussian Processes for Learning Unknown Differential Equations}

We start this section by a brief review of discrete dynamical systems. Given a sequence of $T$ observations, $Y \equiv \mathbf{y}_{1: T}$, discrete dynamical systems, also known as state-space models, are characterized by a first-order Markovian state evolution and an observation equation:

$$
\begin{aligned}
\mathbf{x}_{t+1} & =A(t) \mathbf{x}_{t}+\mathbf{n}(t) \\
\mathbf{y}_{t} & =B(t) \mathbf{x}_{t}+\mathbf{v}(t),
\end{aligned}
$$

where $\mathbf{x}_{t}$ is the $t^{\prime}$ th state of the process of our interest, $A(t)$ is a timevarying state transition matrix, $B(t)$ is the linear observation mapping, and $\mathbf{n}(t)$ and $\mathbf{v}(t)$ stand for noise processes. Although the term state-space originates in the area of control engineering (Kalman, 1960; Shumway et al., 2000), we omit any control inputs for clarity.

A variety of statistical tools such as auto-regressive (AR) models, hidden Markov models and Kalman filters appear as special cases of the above framework. However, due to its linearity and state independence, the transitions $A(t) \mathrm{x}_{t}$ significantly restrict the expressiveness of the dynamical system. This problem can be alleviated by means of nonlinear, black-box function approximations:

$$
\begin{aligned}
\mathbf{x}_{t+1} & =\mathbf{f}\left(\mathbf{x}_{t}, t\right)+\mathbf{n}(t) \\
\mathbf{y}_{t} & =\mathbf{g}\left(\mathbf{x}_{t}, t\right)+\mathbf{v}(t) .
\end{aligned}
$$

Above system is also known as difference equation (Jensen, 2011). Using neural networks for $\mathrm{f}$ and $\mathrm{g}$ leads to so-called recurrent neural networks (RNNs) whose well-known examples include long short-term memory networks (Hochreiter and Schmidhuber, 1997) and gated recurrent units (Chung et al., 2014) (Note that RNNs typically exclude the noise terms but include observations $\mathbf{y}_{t}$ in the dynamics). The unknown $\mathbf{f}$ and $\mathrm{g}$ functions can also be approximated by Gaussian processes, resulting in a series of models (Wang et al., 2006, 2008; Damianou et al., 2011), which are shown to be capable of learning high dimensional video sequences. 
We now give a description of how time series forecasting problem is tackled with AR models and then draw connections with differential equations. Throughout the rest of this chapter, we assume that any dataset of our interest consists of $N$ observed state trajectories $\mathcal{D}=\left\{\left(\mathbf{y}_{0: T}^{(n)}, t_{0: T}^{(n)}\right)\right\}_{n=1}^{N}$ where $\mathbf{y}_{i}:=\mathbf{y}\left(t_{i}\right)$ stands for the $i$ 'th observation within a trajectory observed at time $t_{i}$. We denote the state difference by $\Delta \mathbf{y}_{i}=\mathbf{y}_{i+1}-\mathbf{y}_{i}$ and the time until the next observation by $\Delta t_{i}=t_{i+1}-t_{i}$. Here, we focus on the more generic case in which observations may arrive irregularly in time.

\subsection{Auto-Regressive Dynamics Learning}

In order to learn the state evolution with function approximators, AR models first construct input-output pairs for training. A well-established technique is to extract state and state difference pairs $\left(\mathbf{y}_{i}, \Delta \mathbf{y}_{i}\right)$ and solve the regression problem (Dorffner, 1996; Wang et al., 2006):

$$
\mathbf{y}_{i+1}-\mathbf{y}_{i} \approx \mathbf{f}\left(\mathbf{y}_{i}\right)
$$

with a time-invariant dynamics function. Observe that unlike the formulations introduced earlier, the auro-regressive dynamics are defined in the data space. Learning the dynamics this way implicitly relies on uniform sampling assumption. To see that, imagine the system visits some state twice $\mathbf{y}_{i}=\mathbf{y}_{j}=\mathbf{y}$ at timepoints $t_{i}$ and $t_{j}$. If the observations arrive non-uniformly, i.e., $\Delta t_{i} \neq \Delta t_{j}$, and the system dynamics are different at $\mathbf{y}_{i}$ and $\mathbf{y}_{j}$, then the next states would be different: $\mathbf{y}_{i+1} \neq \mathbf{y}_{j+1}$. Consequently, resulting data pairs $\left(\mathbf{y}_{i}, \Delta \mathbf{y}_{i}\right)$ and $\left(\mathbf{y}_{j}, \Delta \mathbf{y}_{j}\right)$ would have the same input but different targets, which would lead to training issues.

A simple yet effective workaround is "correcting" the state difference with the time increment:

$$
\frac{\mathbf{y}_{i+1}-\mathbf{y}_{i}}{t_{i+1}-t_{i}} \approx \mathbf{f}\left(\mathbf{y}_{i}\right)
$$

We illustrate how time correction resolves the irregular sampling issue on a simulated example, see Figure 3.1 for details.

\subsubsection{Gradient Matching}

A careful examination of (3.2) would reveal that the seemingly ad-hoc time correction term converges to the time differential in the limit. To see this, we rewrite (3.2) by explicitly showing the time dependency:

$$
\frac{\mathbf{y}_{i+1}-\mathbf{y}_{i}}{t_{i+1}-t_{i}}=\frac{\mathbf{y}\left(t_{i}+\Delta t\right)-\mathbf{y}\left(t_{i}\right)}{\Delta t} \underset{\Delta t \rightarrow 0}{\longrightarrow} \frac{d \mathbf{y}\left(t_{i}\right)}{d t_{i}} .
$$

This approach is known as gradient matching since it aims to approximate the unknown state evolution function via the empirical gradients obtained 

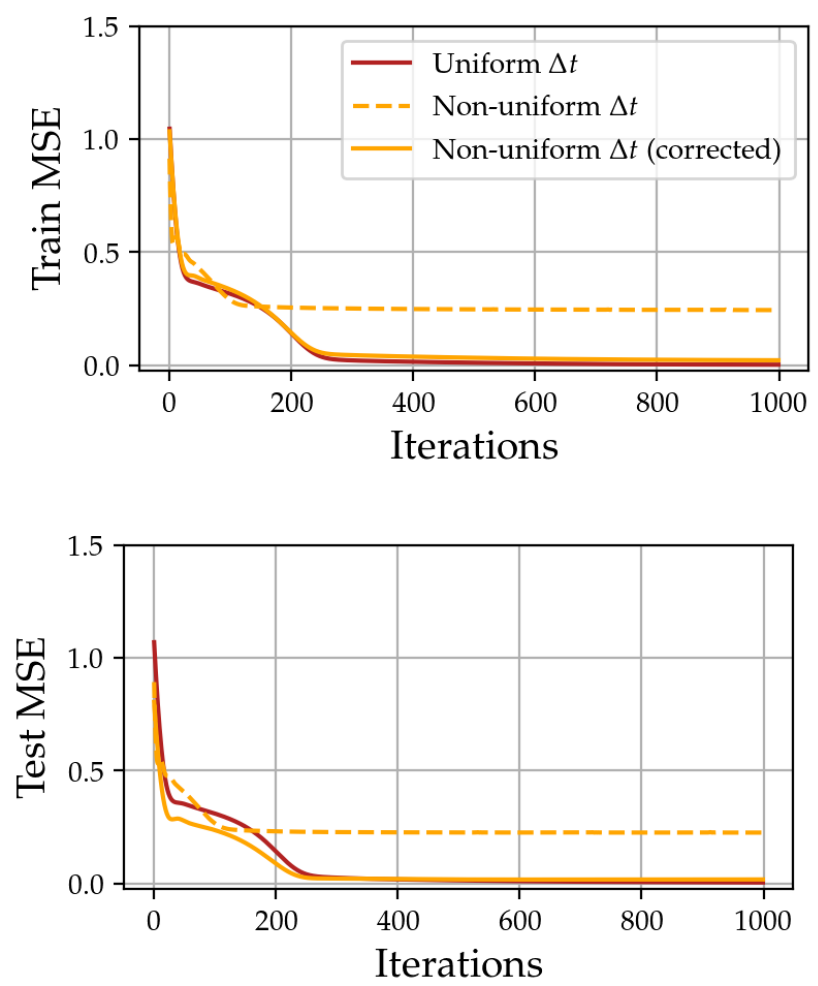

Figure 3.1. An illustration of AR dynamics learning with a neural network approximation f. We generate ten Van der Pol sequences (2.8) for training and five sequences for testing. We consider two scenarios with uniformly (red, $\Delta t=0.1$ ) and irregularly (orange, $\Delta t \sim \mathcal{U}[0,0.4]$ ) sampled data sequences ( $\mathcal{U}$ denoting uniform distribution). We also demonstrate the impact of time correction by training the system with update equations (3.1) and (3.2) on irregularly sampled data. The dashed orange curve shows that the AR model fails to capture the dynamics when trained with irregularly sampled data without time correction. Overlapping solid curves indicate that time correction resolves the issue.

from data pairs (Varah, 1982). Gradient matching has manifested itself as one of the most common strategies to learn ODE systems as it skips the computationally intensive forward integration step (Varah, 1982; Ellner et al., 2002; Ramsay et al., 2007). Nevertheless, gradient matching comes with two fundamental issues: (i) it inevitably leads to wrong differential unless the time difference $\Delta t$ approaches zero, and (ii) noisy observations imply incorrect data pairs $\left(\mathbf{y}_{i}, \Delta \mathbf{y}_{i}\right)$ for dynamics training. Hence, in our Publication I and Publication II, we have taken an alternative approach by computing the costly integration. Before explaining our method, we briefly visit some well-known ODE inference techniques. 


\subsubsection{Related Work}

Several authors have already proposed embedding a parametric differential function within a Bayesian or GP framework (Graepel, 2003; Calderhead et al., 2009; Dondelinger et al., 2013; Wang and Barber, 2014; Macdonald, 2017) (see Macdonald et al. (2015) for a review). GPs have been successfully applied to model linear differential equations as they are analytically tractable (Gao et al., 2008; Raissi et al., 2017). However, conventional ODE modelling can only proceed if a parametric form of the driving function $\mathbf{f}(\cdot)$ is known. Initial work to handle unknown or non-parametric ODE models have been proposed with various limiting approximations. Early works include spline-based smoothing and additive functions $\sum_{j}^{D} f_{j}\left(x_{j}\right)$ to infer gene regulatory networks (De Hoon et al., 2002; Henderson and Michailidis, 2014). Äijö and Lähdesmäki (2009) proposed estimating the unknown nonlinear function with GPs using either finite time differences, or analytically solving the derivative function as a function of only time, $\dot{\mathbf{x}}(t)=\mathbf{f}(t)$ (Äijö et al., 2013). In the technical report of Heinonen and d'Alche Buc (2014) a full vector-valued kernel model $\mathbf{f}(\mathbf{y})$ was proposed, however using a gradient matching approximation.

\subsection{Learning ODEs}

We now explain how unknown time differentials can be learned with GPs. We start by placing a $d>1$ dimensional GP prior on the unknown function $\mathrm{f}: \mathbb{R}^{d} \rightarrow \mathbb{R}^{d}$ (Rasmussen and Williams, 2006)

$$
\mathbf{f}(\mathbf{x}) \sim \mathcal{G P}\left(\mathbf{0}, K\left(\mathbf{x}, \mathbf{x}^{\prime}\right)\right)
$$

where $K\left(\mathbf{x}, \mathbf{x}^{\prime}\right) \in \mathbb{R}^{d \times d}$ is a kernel function. For any set of input points $X=$ $\left[\mathbf{x}_{1}, \mathbf{x}_{2}, \ldots, \mathbf{x}_{N}\right]^{T} \in \mathbb{R}^{N \times d}$, this multi-output GP prior defines a Gaussian distribution over function outputs $F=\left[\mathbf{f}\left(\mathbf{x}_{1}\right), \mathbf{f}\left(\mathbf{x}_{2}\right), \ldots, \mathbf{f}\left(\mathbf{x}_{N}\right)\right]^{T} \in \mathbb{R}^{N \times d}$ :

$$
p(F)=\mathcal{N}(\operatorname{vec}(F) \mid \mathbf{0}, \mathbf{K}(X, X)),
$$

where $\mathbf{K}(X, X)=\left(K\left(\mathbf{x}_{i}, \mathbf{x}_{j}\right)\right)_{i, j=1}^{N} \in \mathbb{R}^{N D \times N D}$ is a block matrix and $\operatorname{vec}(\cdot)$ operator stacks the rows of its argument into a column matrix.

Our goal is to compute the posterior disintribution over $f$ with the following likelihood model

$$
\begin{aligned}
\mathbf{x}(t) & =\mathbf{x}_{0}+\int_{0}^{t} \mathbf{f}(\mathbf{x}(\tau)) d \tau \\
\mathbf{y}_{t} & =\mathbf{x}(t)+\boldsymbol{\epsilon}_{t}, \quad \boldsymbol{\epsilon}_{t} \sim \mathcal{N}(\mathbf{0}, \Omega),
\end{aligned}
$$

where $\mathbf{x}(t)$ and $\mathbf{y}_{t}$ denote a forward simulated state and observation at time $t$, and $\Omega \in \mathbb{R}^{d}$ is the observation noise parameter. As noted by Barber (2014), 
a closed form expression for the state solutions $\mathbf{x}(t)$ is available only if $\mathbf{x}(t)$ follows a Gaussian process and $\mathbf{f}$ is linear with respect to $\mathrm{x}$. Consequently, the majority of existing approaches rely on approximate solutions and sampling state trajectories. In this work, we resort to the sparse GP framework by introducing a set of inducing points $Z=\left[\mathbf{z}_{1}, \mathbf{z}_{2}, \ldots, \mathbf{z}_{M}\right]^{T} \in$ $\mathbb{R}^{M \times d}$ that live in the same space as $\mathbf{x}$, and corresponding output vectors $U=\left[\mathbf{u}_{1}, \mathbf{u}_{2}, \ldots, \mathbf{u}_{M}\right]^{T} \in \mathbb{R}^{M \times d}$ with $\mathbf{u}_{m} \equiv \mathbf{f}\left(\mathbf{z}_{m}\right)$. This approximation leads to a conditional distribution on the function value at any input point, which in turn facilitates explicit forward integration (3.3).

\subsubsection{Time Differential Function}

Due to the formal definition of ODEs, the time differential function in (3.3) needs to be continuous whereas we introduced a (conditional) stochastic process over the function, i.e., $p\left(\mathbf{f}\left(\mathbf{x}^{*}\right) \mid Z, U\right)$. To resolve this, we propose to discard the variance induced by the distribution $p\left(\mathbf{f}\left(\mathbf{x}^{*}\right) \mid Z, U\right)$ :

$$
\mathbf{f}\left(\mathbf{x}^{*}\right) \mid Z, U \triangleq \mathbf{K}_{\boldsymbol{\theta}}\left(\mathbf{x}^{*}, Z\right) \mathbf{K}_{\boldsymbol{\theta}}(Z, Z)^{-1} \operatorname{vec}(U),
$$

where $\boldsymbol{\theta}$ denotes the kernel parameters. We opt for the simplest identity decomposable kernel as our kernel function:

$$
K_{\boldsymbol{\theta}}\left(\mathbf{x}, \mathbf{x}^{\prime}\right) \triangleq k_{\boldsymbol{\theta}}\left(\mathbf{x}, \mathbf{x}^{\prime}\right) \cdot I_{d}
$$

where $I_{d}$ denotes a $d \times d$ identity matrix. Diagonal elements of the kernel matrix are given by a the following scalar-valued squared exponential kernel, the de-facto standard kernel for GPs:

$$
k_{\boldsymbol{\theta}}\left(\mathbf{x}, \mathbf{x}^{\prime}\right)=\sigma_{f}^{2} \exp \left(-\frac{1}{2} \sum_{j=1}^{d} \frac{\left(x_{j}-x_{j}^{\prime}\right)^{2}}{\ell_{j}^{2}}\right)
$$

where $\sigma_{f}$ is known as signal variance and $\ell_{j}$ denotes dimension-wise lengthscale parameter, i.e., $\boldsymbol{\theta}=\left\{\sigma_{f}, \ell_{1}, \ldots, \ell_{d}\right\}$. We name our model $n p O D E$, abbreviating non-parametric ODEs, and illustrate it in Figures 3.2 and 3.3. Please note that non-parametric refers to our model's ability to approximate any ODE system without having access to its parameters or functional form of the time differential.

\subsubsection{MAP Inference for npODE}

In this section, we describe the inference task for a single input data sequence $Y \equiv \mathbf{y}_{0: T}$ without loss of generality. As described in (3.3-3.4), the likelihood is given by a Gaussian observation model with mean being the forward simulated states $\mathbf{x}(t)$. For simplicity, we assume a diagonal noise covariance $\Omega=\operatorname{diag}\left(\omega_{1}, \cdots, \omega_{d}\right)$ to be learned. Due to the noisy observation 
(a) Vector field

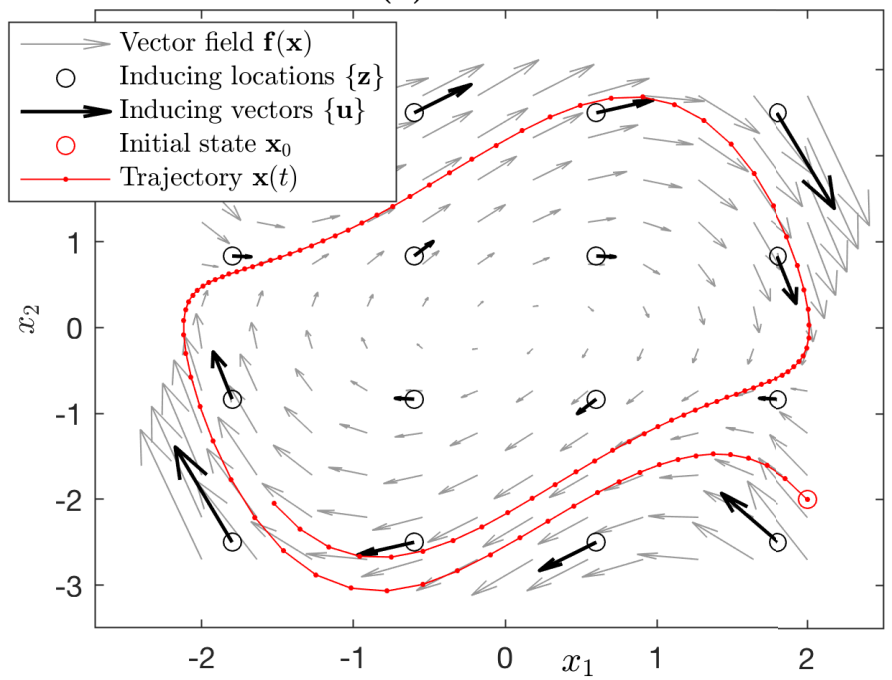

(b) ODE solution

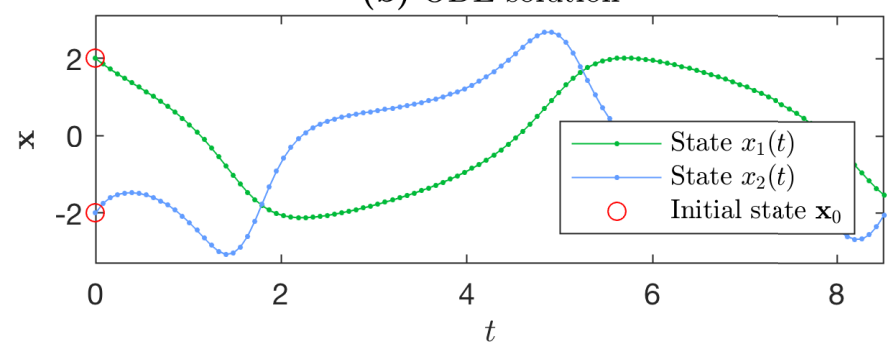

Figure 3.2. $2 D$ illustration of npODE on Van der Pol oscillator (the figure is taken from Publication I). (a) Above panel demonstrates the model in the phase space, where each axis corresponds to an observed dimension. Black circles and arrows denote the inducing locations $Z$ and vectors $U$. Grey arrows in the background are computed by interpolating via (3.5). The red trajectory is computed by integrating the resulting system starting from the red circle. (b) The states plotted over time.

assumption, the initial value $\mathrm{x}_{0}$ also appears as a free parameter. Overall, the posterior distribution becomes

$$
\begin{aligned}
p\left(Z, U, \mathbf{x}_{0}, \boldsymbol{\theta}, \Omega \mid Y\right) & \propto p\left(Y \mid \mathbf{x}_{0}, U, \Omega, Z, \boldsymbol{\theta}\right) p(U \mid Z, \boldsymbol{\theta}) p\left(Z, \mathbf{x}_{0}, \boldsymbol{\theta}, \Omega\right) \\
& \propto \prod_{i=0}^{T} \mathcal{N}\left(\mathbf{y}_{i} ; \mathbf{x}\left(t_{i}\right), \Omega\right) \mathcal{N}\left(\operatorname{vec}(U) \mid \mathbf{0}, \mathbf{K}_{\boldsymbol{\theta}}(Z, Z)\right),
\end{aligned}
$$

where we assume a Gaussian process prior on the inducing vectors and non-informative priors for the remaining variables.

Inducing locations To simplify the learning, we propose to place inducing points $Z$ on a fixed and sufficiently dense grid. The grid locations are chosen to cover the training data sequences. We have experimentally observed that learning the locations complicates gradient descent based optimization 
routines and usually leads to worse test performance compared to learning with a fixed grid.

Whitened inducing vectors As another measure to stabilize learning, we aim to reduce the correlation between inducing points by an invertible transformation known as whitening Kuss and Rasmussen (2005). Whitening relies on transforming the inducing variables using the Cholesky decomposition of the covariance matrix $\mathbf{L}_{\boldsymbol{\theta}} \mathbf{L}_{\boldsymbol{\theta}}^{T}=\mathbf{K}_{\boldsymbol{\theta}}(Z, Z)$ as follows:

$$
\operatorname{vec}(U)=\mathbf{L}_{\boldsymbol{\theta}} \operatorname{vec}(\widetilde{U}), \quad \operatorname{vec}(\widetilde{U})=\mathbf{L}_{\boldsymbol{\theta}}^{-1} \operatorname{vec}(U)
$$

In turn, $\widetilde{U}$ appears as the model parameters instead of $U$, and the prior distribution becomes $p(\widetilde{U})=\mathcal{N}(0, I)$. The whitened variables $\widetilde{U}$ can easily be projected on the kernel manifold $\mathbf{L}_{\boldsymbol{\theta}}$ to obtain the inducing vectors $U$.

MAP estimation Since full posterior inference of our model is difficult, we opt for computing the MAP estimates for the initial state $\mathrm{x}_{0}$, whitened inducing vectors $\widetilde{U}$, and noise variances $\Omega$ instead of inferring the full posterior:

$$
\underset{\mathbf{x}_{0}, \widetilde{U}, \Omega}{\arg \max } \sum_{i=0}^{T} \log \mathcal{N}\left(\mathbf{y}_{i} ; \mathbf{x}\left(t_{i}\right), \Omega\right)+\log \mathcal{N}(\operatorname{vec}(\widetilde{U}) ; 0, I) .
$$

Note that we choose the kernel hyperparameters $\theta$ by cross-validation, which helps alleviating the overfitting problem. The gradients of the above system are computed via the sensitivity equations (2.13). In all forward simulations, we use RK45 solver (Dormand and Prince, 1980).

\subsection{Learning SDEs}

We now turn out attention to the cases where the dynamics are inherently stochastic. Perhaps the simplest example of such cases is the flow of particles suspended in liquid, which is explained using the friction and random forces caused by random collisions of the particles (Langevin, 1908; Lemons and Gythiel, 1997). The randomness is typically expressed via the non-differentiable Brownian motion, making ODEs an unsuitable approach. Therefore, in this section, we extent npODE to handle such nondifferentiable cases by means of stochastic differential equations (SDEs).

For conciseness, we first re-introduce the Itô integral which was defined in (2.2.2):

$$
\mathbf{x}(t)=\mathbf{x}_{0}+\int_{0}^{t} \mathbf{f}(\mathbf{x}(\tau)) d \tau+\int_{0}^{t} \sigma(\mathbf{x}(\tau)) d B_{\tau} .
$$

Above differential equation differs from the ODE definition (3.3) due to the second integral. Thus, we propose to build upon the npODE framework by 

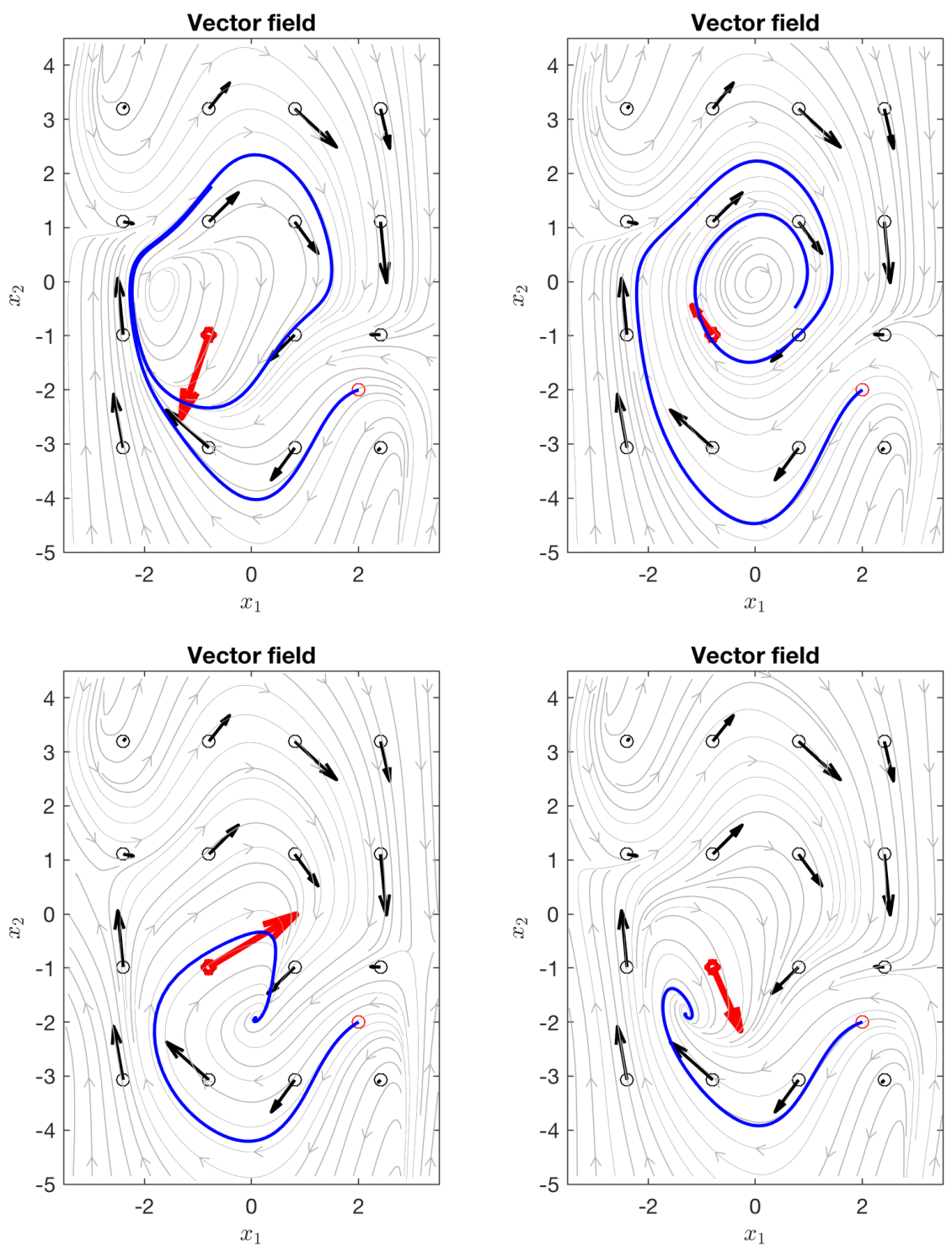

Figure 3.3. We illustrate how a single inducing vector influences the resulting system on the Van der Pol oscillator. Across all panels, only the inducing vector shown by red vary. The gray streams denote the vector field given by the kernel interpolation (3.5), which substantially differ near the red arrow. This locally different behaviour immediately translates into unique blue state trajectories that are computed by forward simulating the ODE system by (3.3). 
introducing a second set of inducing vectors to approximate the diffusion function $\sigma(\cdot)$ :

$$
\sigma\left(\mathbf{x}^{*}\right) \mid Z, U \triangleq k\left(\mathbf{x}^{*}, Z\right) k(Z, Z)^{-1} U_{\sigma} .
$$

The new set of "diffusion" inducing vectors, $U_{\sigma}=\left[u_{1}^{\sigma}, \ldots, u_{M}^{\sigma}\right] \in \mathbb{R}^{M}$ with $u_{m}^{\sigma} \equiv \sigma\left(\mathbf{z}_{m}\right)$, share the same inducing locations $Z$ with the "drift" inducing vectors, which we rename as $U_{\mathbf{f}}$ for clarity. Observe that the proposed diffusion function estimator is one-dimensional; however, it can be easily extended to higher dimensions by defining multiple GPs as in previous section. We name our approach $n p S D E$.

MAP estimation Similar to npODE, our goal is to compute the MAP estimate of the parameters $U_{\mathbf{f}}, U_{\sigma}$ and $\Omega$ :

$$
\underset{U_{\mathbf{f}}, U_{\sigma}, \Omega}{\arg \max } p\left(U_{\mathbf{f}}, U_{\sigma}, \Omega \mid Y\right) \propto p\left(Y \mid U_{\mathbf{f}}, U_{\sigma}, \Omega\right), p\left(U_{\mathbf{f}}\right) p\left(U_{\sigma}\right) p(\Omega)
$$

where the GP priors defined in (3.7) are placed over $U_{\mathrm{f}}$ and $U_{\sigma}$, and a non-informative prior over $\Omega$. Kernel hyperparameters $\boldsymbol{\theta}$ and inducing locations $Z$ are chosen as described in the previous section (we drop them from the conditioning notation for conciseness). Finally, we opt for setting the initial value of the integral to the first observation: $\mathbf{x}_{0} \equiv \mathbf{y}_{0}$. Note that this simple choice works well in practice only if the data contains little amount of noise. Alternatively, our framework can be extended to include the initial value $\mathrm{x}_{0}$ as a parameter.

Likelihood Since each different realization of the stochastic process leads to a different state solution, our likelihood model should be expressed in terms of the state solution distributions $p\left(\mathbf{x}\left(t_{i}\right) \mid U_{\mathbf{f}}, U_{\sigma}\right)$. Thus, we propose to learn the underlying system to induce state distributions with high expected likelihood:

$$
p\left(Y \mid U_{\mathbf{f}}, U_{\sigma}, \Omega\right)=\prod_{i=0}^{T} \mathbb{E}_{p\left(\mathbf{x}\left(t_{i}\right) \mid U_{\mathbf{f}}, U_{\sigma}\right)}\left[\mathcal{N}\left(\mathbf{y}_{i} \mid \mathbf{x}\left(t_{i}\right), \Omega\right)\right]
$$

Monte Carlo sampling The state solutions $p\left(\mathbf{x}\left(t_{i}\right) ; U_{\mathbf{f}}, U_{\sigma}\right)$ do not admit tractable closed-form expressions with nonlinear drift and diffusion functions. A simple yet efficient approximation can be obtained by Monte Carlo sampling:

$$
p\left(Y \mid U_{\mathbf{f}}, U_{\sigma}, \Omega\right) \approx \prod_{i=0}^{T} \frac{1}{L} \sum_{l=1}^{L} \mathcal{N}\left(\mathbf{y}_{i} \mid \mathbf{x}_{i}^{(l)}, \Omega\right), \quad \mathbf{x}_{i}^{(l)} \sim p\left(\mathbf{x}\left(t_{i}\right) \mid U_{\mathbf{f}}, U_{\sigma}\right)
$$

where we use the shorthand notation $\mathbf{x}_{i} \equiv \mathbf{x}\left(t_{i}\right)$. State solution samples $\mathbf{x}_{i}^{(l)}$ can be obtained by first drawing a Brownian motion sample and then numerically integrating (3.9). We used the simplest Euler-Maruyama solver given in (2.11) for numerical integration. Note that above stochastic likelihood estimate turns out to be a kernel density estimator with 
Gaussian bases. We experimentally observed that the simple Monte Carlo approximation to state solution distributions $p\left(\mathbf{x}\left(t_{i}\right) ; U_{\mathbf{f}}, U_{\sigma}\right)$ works well in practice. Alternatively, the expectation can be approximated using the sequential importance sampling method presented in Sarkka (2006) if the diffusion is constant or using more complicated particle based MCMC techniques described in Botha et al. (2021).

Gradient computation The gradient of the Monte Carlo approximated log-likelihood (3.11) is

$$
\frac{d}{d U} \sum_{i=0}^{T} \log \frac{1}{L} \sum_{l=1}^{L} \mathcal{N}\left(\mathbf{y}_{i} \mid \mathbf{x}_{i}^{(l)}, \Omega\right)=\sum_{i=0}^{T} \frac{\sum_{l=1}^{L} \frac{\partial \mathcal{N}\left(\mathbf{y}_{i} \mid \mathbf{x}_{i}^{(l)}, \Omega\right)}{\partial \mathbf{x}} \frac{d \mathbf{x}_{i}^{(l)}}{d U}}{\sum_{l=1}^{L} \mathcal{N}\left(\mathbf{y}_{i} \mid \mathbf{x}_{i}^{(l)}, \Omega\right)}
$$

where $\mathbf{x}_{i}^{(l)}$ follows numerical integration and $U$ represents either set of inducing vectors. Above, the only difficult term $\frac{d \mathbf{x}_{i}^{(l)}}{d U}$ can be computed by slightly modifying forward sensitivities, see Publication II for a detailed description. Also, note that modern automatic differentiation frameworks conveniently utilize the same Brownian motion (or random numbers, in general) during the forward and backward pass, which leads to accurate gradients.

To sum up, our npODE and npSDE rely on four main principles: estimating the unknown functions with black-box function approximators, MAP estimation of the parameters, explicit integration to compute state solutions, and sensitivity equations for gradient computation. Unlike the npODE approach, in which whitened transformations of inducing points appear as parameters, we choose simplify npSDE framework by optimizing the inducing values themselves. Finally, npSDE integration starts from the initial observation $\mathbf{y}_{0}$ while npODE treats the initial values as unknowns.

\subsection{Experiments with npODE and npSDE}

In this section, we illustrate our non-parametric differential equation systems on simulated and real data experiments. For simulated experiments, we consider three well-known ODE systems and one SDE system whose details are presented in the respective subsections. Our goal is to show that black-box modeling of parametric systems leads to accurate estimation of the dynamics. The real data experiment is conducted on a benchmark dataset of human motion capture data from the Carnegie Mellon University motion capture (mocap) database ${ }^{1}$. Since no parametric differential equation exists for human motion, this experiment demonstrates how our model can estimate realistic, unknown dynamics from noisy observations. Please note that additional results can be found in Publication I and II.

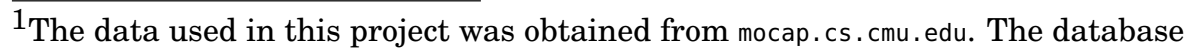
was created with funding from NSF EIA-0196217.
} 

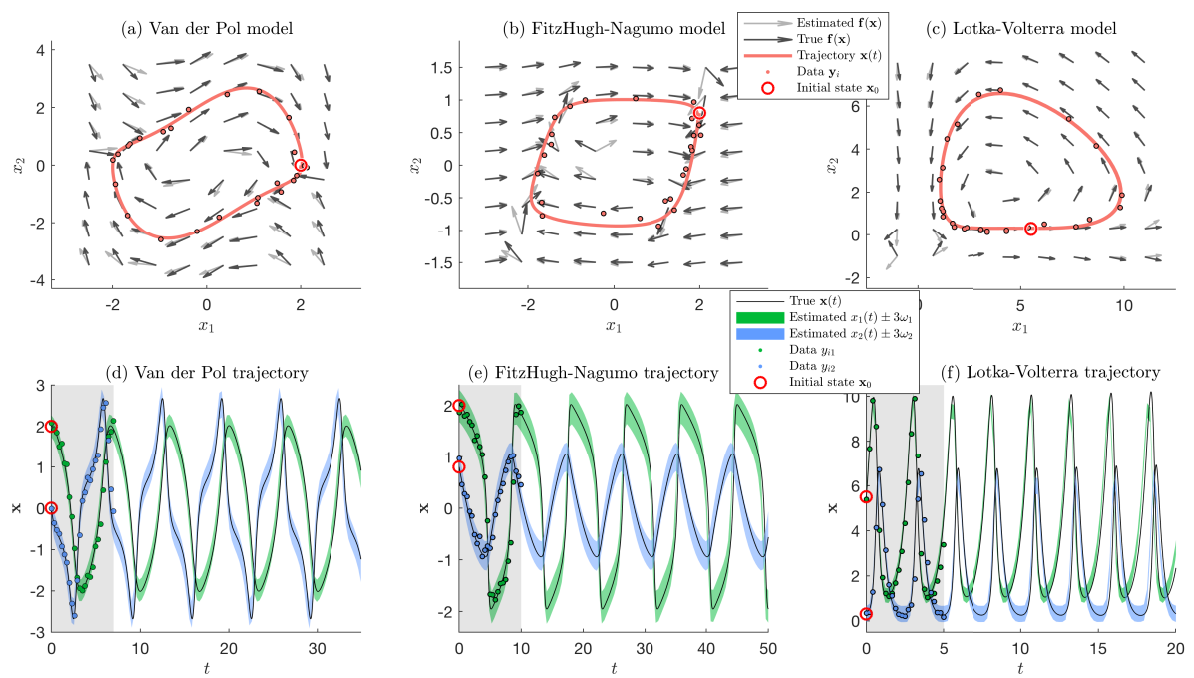

Figure 3.4. Estimated dynamics from Van der Pol, FitzHugh-Nagumo and Lotka-Volterra systems. The top part (a-c) shows the noisy data points (red circles), limit cycles (red trajectory), learned vector field (grey arrows), and the true vector field (black arrows). The bottom panels (d-f) show the training data over time (circles in the grey region), true future cycles (black lines), forecasted future cycles \pm 3 standard deviation (shaded regions). Note that the figure is taken from Publication I.

\subsubsection{Simulated ODE Systems}

We consider three simulated differential systems: the Van der Pol (VDP), FitzHugh-Nagumo (FHN) and Lotka-Volterra (LV) oscillators of the following form:

$$
\begin{array}{rll}
\text { VDP : } & \dot{x}_{1}=x_{2} & \dot{x}_{2}=\left(1-x_{1}^{2}\right) x_{2}-x_{1} \\
\text { FHN : } & \dot{x}_{1}=3\left(x_{1}-\frac{x_{1}^{3}}{3}+x_{2}\right) & \dot{x}_{2}=\frac{0.2-3 x_{1}-0.2 x_{2}}{3} \\
\text { LV : } & \dot{x}_{1}=1.5 x_{1}-x_{1} x_{2} & \dot{x}_{2}=-3 x_{2}+x_{1} x_{2} .
\end{array}
$$

We generate five training sequences of length 25 for each oscillator. Each VDP and FHN trajectory contains one cycle whereas LV trajectories make up 1.7 cycles. We also add observation noise with variance $\sigma_{n}^{2}=0.1^{2}$. The inducing points are placed on a $6 \times 6$ fixed grid. Figure 3.4 demonstrates our findings. We observe that $\mathrm{npODE}$ is able to accurately estimate the vector field, especially near the data points. Consequently, it can successfully forecast up to 8 future cycles.

\subsubsection{Simulated SDE System}

We now illustrate the merits of our npSDE on a simulated SDE system in which the drift function is given by the Van der Pol equations and the diffusion is proportional to a Gaussian density. The true and unknown 

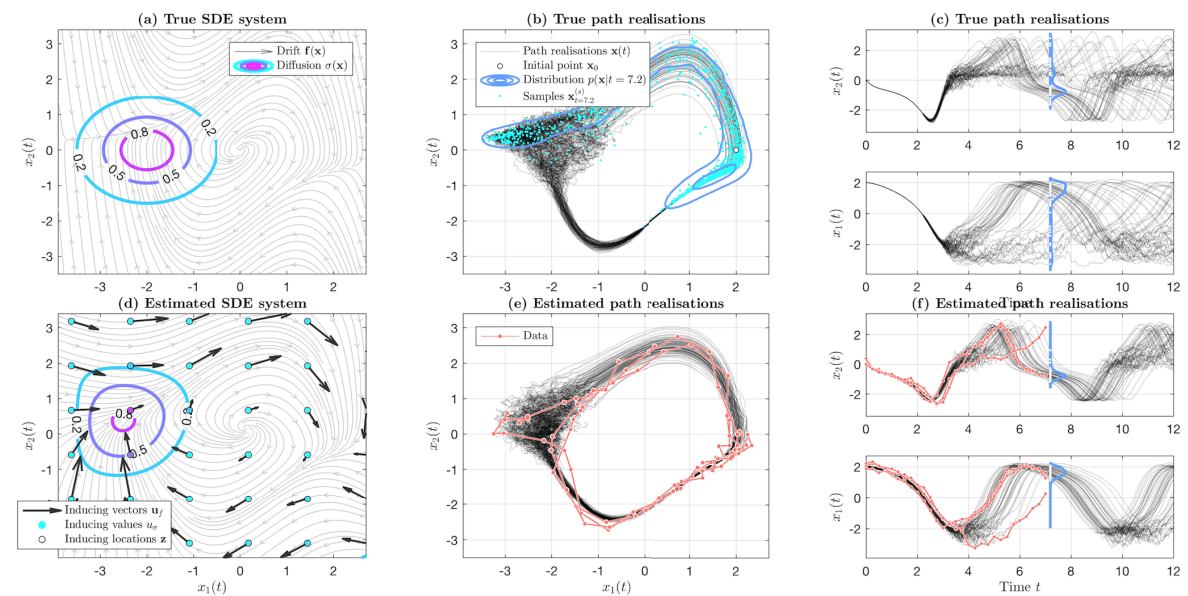

Figure 3.5. (a) A Van der Pol oscillator with local diffusion function. (b) The phase panel of the trajectory samples. The blue counter plot represents the state density $p\left(\mathbf{x}(7.2) ; U_{\mathbf{f}}, U_{\sigma}\right)$ at $t=7.2$ (c) The same trajectory samples plotted over time. Blue curves denote the marginal distribution of each state component at $t=7.2$. (d) Estimated drift and diffusion functions. (e) Three noisy input trajectories for training (red) and forward simulated paths from the estimated model (black). (f) Data trajectories (red) and estimated path samples plotted over time (black) along with the same state marginals in (c) (blue). Note that the figure is taken from Publication II.

vector field and the diffusion "hot-spot" is illustrated in Figure 3.5a. The blue contour plot in (b) represents the state density $p(\mathbf{x}(t))$ at $t=7.2$, which is due to the hot-spot. We generate ten training sequences of length 25 , three of which are plotted in (e) and (f).

Figure 3.5d shows the drift and diffusion estimates, which match the unknown true functions in (a). Trajectory samples from the true (b) and estimated systems (e) also overlap. This is more apparent when the marginal state distributions are plotted over time (c-f). As expected, the variance of sampled trajectories grows near the hot-spot, and they are almost smooth far from the hot-spot.

\subsubsection{Motion Capture Experiments}

Next, we evaluate our model on a benchmark real-world dataset of walking sequences. The dataset consists of 50-dimensional pose measurements from walking people, where each measurement comes from a sensor attached to subjects' bodies (Wang et al., 2008). After pre-processing the data as described in Wang et al. (2008), we obtain a training dataset of 43 trajectories, each containing roughly 100 measurements. Following Wang et al. (2006) and Damianou et al. (2011), the dataset is projected into a three dimensional latent space by principle component analysis (PCA), which helps eliminating the highly-correlated features.

For ODE learning task, PCA embedding of each training sequence is fitted separately since walking sequences follow subject-specific styles. 
(a)

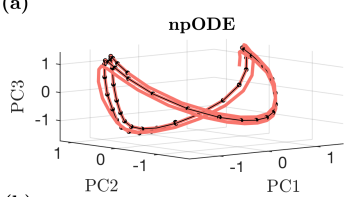

(b)
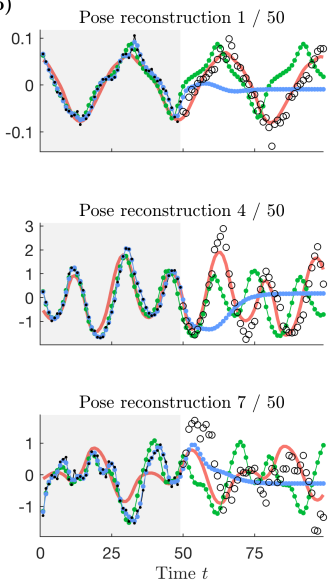

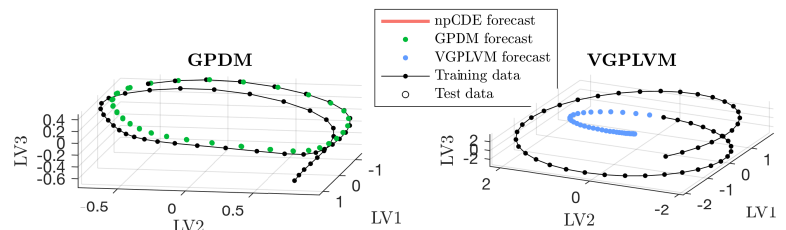

Pose reconstruction $2 / 50$
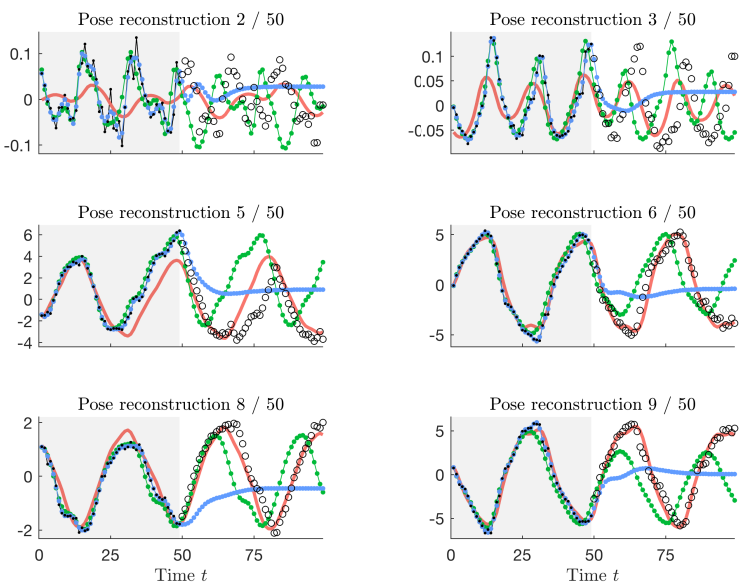

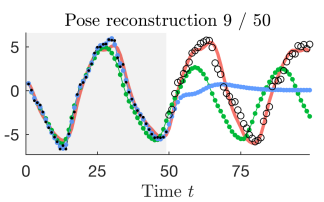

Figure 3.6. An illustration of npODE and competing methods on mocap experiment (the figure is taken from Publication I). (a) Three dimensional latent space in which dynamics learning is performed. Black points represent the training data and colored lines are future forecasts. (b) Latent sequences are reconstructed into the original fifty dimensional space via inverse PCA mapping. The grey region shows the training data.

We use the first half of a sequence for training and cross validation, and compute the prediction error on the second half. As baselines, we consider GP based state space models GPDM (Wang et al., 2006) and variational GPLVM (Damianou et al., 2011). Overall, our method's root mean squared error (RMSE) on future time points is 4.52, while GPDM and VGPLVM achieve 4.94 and $8.74 \mathrm{RMSE}$, respectively. A comparison of the three methods on a single sequence is presented in Figure 3.6.

To demonstrate npSDE on real-world data scenarios, we follow the same experiment setup with Wang et al. (2008), and collect four walking sequences from different subjects into a single dataset. Our goal is to learn a drift function that models the walking dynamics and a diffusion function that explains the discrepancies among walking styles. We visualize the inferred drift fit and the density of the sample paths in Figure 3.7. We conclude that our model is capable of learning drift and diffusion functions that match arbitrary data sequences. 

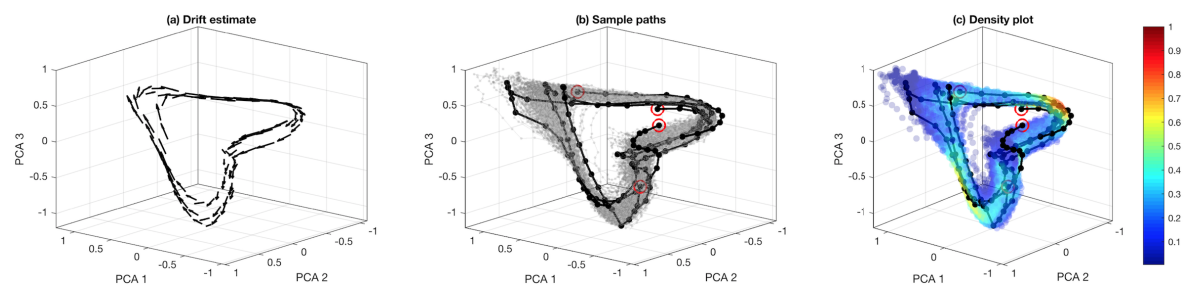

Figure 3.7. npSDE model fits on mocap data (the figure is taken from Publication II). (a) Shared drift estimate learned from walking data of four subjects. (b) Estimated sample paths. (c) Density plots of the sample paths. Four observed trajectories are shown as black lines in (b-c), with red circles denoting the initial state. 


\section{Continuous-Time Bayesian Modelling for High Dimensional Sequences}

In the previous chapter, we explained how continuous-time phenomena can be learned by means of Gaussian processes. As demonstrated by the simulated system experiments, the framework is capable of inferring unknown systems given noisy state measurements. Nevertheless, the model suffers from certain limitations which we aimed to address in $\mathrm{ODE}^{2} \mathrm{VAE}$, the method proposed in our Publication III. In the next section, we examine the limitations of $\mathrm{npODE}$, which naturally motivate the ideas behind $\mathrm{ODE}^{2} \mathrm{VAE}$. We then describe our proposed variational framework that relies on variational auto-encoders and Bayesian neural networks. The chapter is concluded with the experimental evaluations.

\subsection{Limitations of npODE}

Since the methodologies presented in the previous chapter are analogous, the discussion in this section is limited to npODE (while the same arguments apply to npSDE as well). In the following, we describe five shortcomings of npODE. A complete list of differences between npODE and $\mathrm{ODE}^{2} \mathrm{VAE}$ is given in Table 4.1.

\subsubsection{Modeling Space}

npODE dynamics specified in (3.3-3.4) are chosen to live in the same space as the observations. Nonetheless, as opposed to the model description, walking dynamics were learned in a $3 D$ latent space onto which the dataset was projected by PCA. npODE's success on this task can be partly attributed to the fact that sensor measurements are highly correlated, making a linear projection sufficient for our purposes. However, in more complicated problems, linear mappings may not reflect the underlying structure in the data. Lawrence and Hyvärinen (2005) indeed show that a non-linear variant of PCA, known as Gaussian process latent variable model, better structure the latent space, which in turn leads to more 
Table 4.1. A summary of the differences between NPODE and $\mathrm{ODE}^{2} \mathrm{VAE}$.

\begin{tabular}{lll}
\hline & NPODE & ODE $^{2}$ VAE \\
\hline Modeling space & Data space & Latent space \\
Dynamics approximator & Gaussian process & Bayesian neural net. \\
Inference & MAP inference & Variational inference \\
Order of the dynamics & First & Second \\
Gradient computation & Sensitivity equations & Adjoint method \\
Data modality & States & States/images \\
\hline
\end{tabular}

interpretable embeddings and much smaller latent classification error.

\subsubsection{Learning Second-Order Systems}

Classical mechanics describes the motions of everyday objects by the language of differentials and integrals. Not only the simplest mechanics formulations, e.g. Newton's laws, but also more sophisticated ones such as Lagrangian and Hamiltonian mechanics rely on higher order derivatives. Without diving into the details of such formulations, we define a secondorder ODE system in terms of position $\mathbf{s}(t)$ and velocity $\mathbf{v}(t)$ components:

$$
\begin{aligned}
\dot{\mathbf{s}}(t) & =\frac{d \mathbf{s}(t)}{d t}=\mathbf{v}(t) \\
\dot{\mathbf{v}}(t) & =\frac{d \mathbf{v}(t)}{d t}=\mathbf{f}(\mathbf{s}(t), \mathbf{v}(t), \mathbf{u}(t), t, \mathbf{C})
\end{aligned}
$$

where the equations represent velocity and acceleration, respectively. Being the time derivative of velocity, acceleration (4.2) is generally an arbitrary function of time $t$, position $\mathbf{s}(t)$, velocity $\mathbf{v}(t)$, force $\mathbf{u}(t)$, and possibly other system-specific variables $\mathbf{C}$ such as mass and friction. We conjecture that above formulation is more suitable for modeling systems that are expressed via the laws of motion.

\subsubsection{Scalability of the Dynamics}

Being a kernel method, sparse Gaussian processes compute function outputs as a locally weighted average of other data points, or inducing values. When the kernel function is stationary, as the squared exponential kernel used in npODE, learning non-stationarities becomes too difficult. The problem may be relieved by placing a large number of inducing points near the non-stationary regions; however, npODE framework assumes a fixed grid, and knowing such regions rather than inferring would be a restrictive assumption. Second, kernel values quickly diminish as the state dimensionality increases, famously known as curse of dimensionality. 
This necessitates a growing set of inducing points, which would in turn significantly slow down the computations.

\subsubsection{Overfitting}

Learning in npODE framework relies on MAP estimation instead of marginalizing out the unknown quantities. Since the marginal likelihood is not available, choosing the correct model complexity (without overfitting) was attained by cross-validation, which requires repeating the optimization procedure several times from scratch. Furthermore, MAP estimates consist of likelihood and prior terms, which are responsible for data fitting and regularization, respectively. Nonetheless, npODE posterior distribution (3.6) does not place a prior on the inducing locations and initial value, making the model vulnerable to overfitting.

\subsubsection{Scalability of the Optimization}

The gradient of npODE loss was computed using forward sensitivities (2.12). The method requires solving a $d P$-dimensional ODE system, where $d$ and $P$ denote the state dimensionality and number of parameters in the differential function. Therefore, forward sensitivities can handle systems with $P \leq 500$ (Rackauckas et al., 2018). Scalability was not a problem in the context of npODEs since $P$ typically varies in $[50,300]$. However, with larger scale problems and over-parameterized time differential approximations such as neural networks, gradient computation becomes a huge computational bottleneck.

\subsection{Proposed Model}

Building upon the observations in the previous section, we propose to infer continuous-time, second-order latent trajectories that live in a smaller space. For this, we consider a generative model with three components:

(i) Second order dynamics defined by an acceleration field

(ii) A distribution for the initial position $p\left(\mathbf{s}_{0}\right)$ and velocity $p\left(\mathbf{v}_{0}\right)$ in the latent space

(iii) A likelihood model $p(\mathbf{x} \mid \mathbf{s})$ that maps latent positions into high-dimensional observations,

which can be written more concretely as follows:

$$
\begin{aligned}
& \mathbf{s}_{0} \sim p\left(\mathbf{s}_{0}\right) \\
& \mathbf{v}_{0} \sim p\left(\mathbf{v}_{0}\right)
\end{aligned}
$$




$$
\begin{aligned}
& \mathbf{s}_{t}=\mathbf{s}_{0}+\int_{0}^{t} \mathbf{v}_{\tau} d \tau \\
& \mathbf{v}_{t}=\mathbf{v}_{0}+\int_{0}^{t} \mathbf{f}_{\text {true }}\left(\mathbf{s}_{\tau}, \mathbf{v}_{\tau}\right) d \tau \\
& \mathbf{x}_{i} \sim p\left(\mathbf{x}_{i} \mid \mathbf{s}_{i}\right) \quad i \in[0, T]
\end{aligned}
$$

As in npODE, we consider a parametric approximation to the unknown latent differential function $f_{\text {true }}$. Due to the scalability issues discussed before, we opt for a neural network (NN) approximation instead of GPs. As in other generative models, our primary optimization objective is to compute the marginal likelihood by integrating out the latent position and velocity variables. With non-linear time differential or likelihood functions, marginalization becomes intractable and we resort to variational approximation. In particular, we turn our attention to VAE, which is proven useful for learning highly complicated latent mappings (Kingma and Welling, 2014; Rezende et al., 2014), and Bayesian neural networks.

Variational auto-encoder We first shortly remind the idea behind VAE (please see Subsection 2.3.1 for a more rigorous treatment). Given a data point $\mathbf{x}$ and an intractable posterior density $p(\mathbf{z} \mid \mathbf{x})$ over the corresponding latent variable $\mathrm{z}$, the encoder objective is to approximate the posterior with a variational family $q_{\psi}(\mathbf{z} \mid \mathbf{x})$ while the decoder learns the inverse mapping $p_{\gamma}(\mathbf{x} \mid \mathbf{z})$, which corresponds to the likelihood model. Typically, both the encoder and decoder are parameterized by deep neural networks (usually CNNs in image based applications and MLPs otherwise).

Although VAE is a rather recently popularized tool presented mainly in the context of variational inference, encoder-decoder type architectures have been known for decades, e.g., a non-probabilistic VAE variant called autoassociator was first proposed for dimensionality reduction (Cottrell, 1985). In the context of latent ODE modeling, we utilize the encoder to extract a latent representation only for the initial value of a trajectory while the decoder maps all forward simulated latent states into data space.

Bayesian neural networks As mentioned in the previous section, npODE lacks a principled approach to deal with overfitting. When the GP time differential approximation is replaced with a $\mathrm{NN}$, the resulting model would still be susceptible to overfitting since vanilla multi-layer perceptron described in Section 2.1.1 provides no means for handling uncertainty. Therefore, we choose to treat the NN parameters $\mathcal{W}$ as random variables, which in turn corresponds to modeling unknown dynamics with a BNN $\mathbf{f}_{\mathcal{W}}\left(\mathbf{s}_{t}, \mathbf{v}_{t}\right)$. 


\subsubsection{Approximate Posterior}

We now describe our variational framework from the first principles. For notational clarity, we combine the latent position and velocity components into a single vector $\mathbf{z}_{t}:=\left(\mathbf{s}_{t}, \mathbf{v}_{t}\right)$ and propose the following approximation for the unknown quantities:

$$
q\left(\mathcal{W}, \mathbf{z}_{0: T} \mid \mathbf{x}_{0: T}\right)=q(\mathcal{W}) q_{\text {enc }}\left(\mathbf{z}_{0} \mid \mathbf{x}_{0: T}\right) q_{\text {ode }}\left(\mathbf{z}_{1: T} \mid \mathbf{z}_{0}, \mathcal{W}\right)
$$

The first term on the rhs defines the mean-field approximate posterior for the dynamics approximation:

$$
q(\mathcal{W})=\mathcal{N}(\mathcal{W} \mid \mathbf{m}, s \mathbf{I})
$$

The second term is based on the amortized VI idea of VAE, where $q_{\text {enc }}$ is given by NN encoders. Similar to original VAE formulation (Kingma and Welling, 2014), latent representations have a diagonal covariance matrix:

$$
\begin{aligned}
q_{\mathrm{enc}}\left(\mathbf{z}_{0} \mid \mathbf{x}_{0: T}\right) & \equiv q_{\mathrm{enc}}\left(\left(\begin{array}{c}
\mathbf{s}_{0} \\
\mathbf{v}_{0}
\end{array}\right) \mid \mathbf{x}_{0: T}\right) \\
& =\mathcal{N}\left(\left(\begin{array}{c}
\boldsymbol{\mu}_{\mathbf{s}}\left(\mathbf{x}_{0}\right) \\
\boldsymbol{\mu}_{\mathbf{v}}\left(\mathbf{x}_{0: m}\right)
\end{array}\right),\left(\begin{array}{cc}
\operatorname{diag}\left(\boldsymbol{\sigma}_{\mathbf{s}}\left(\mathbf{x}_{0}\right)\right) & \mathbf{0} \\
\mathbf{0} & \operatorname{diag}\left(\boldsymbol{\sigma}_{\mathbf{v}}\left(\mathbf{x}_{0: m}\right)\right)
\end{array}\right)\right),
\end{aligned}
$$

where $\mu_{\mathrm{s}}, \boldsymbol{\mu}_{\mathrm{v}}, \boldsymbol{\sigma}_{\mathrm{s}}, \boldsymbol{\sigma}_{\mathrm{v}}$ are the encoding NNs. Note that the latent position representation is computed conditioned only on the initial data point while the higher-order velocity information is extracted from the first $1<m \leq T$ data points.

Instantaneous change of variables The last term in (4.3) describes how the density evolves as the initial latent point $\mathbf{z}_{0}$ is forward integrated. For this, we first express our second-order system as a first order ODE as described in Section 2.2.1:

$$
\begin{aligned}
\mathbf{z}_{N} & =\mathbf{z}_{0}+\int_{0}^{t_{N}} \tilde{\mathbf{f}}_{\mathcal{W}}\left(\mathbf{z}_{\tau}\right) d \tau \\
\tilde{\mathbf{f}}_{\mathcal{W}}\left(\mathbf{z}_{t}\right) & \equiv\left(\begin{array}{c}
\mathbf{v}_{t} \\
\mathbf{f}_{\mathcal{W}}\left(\mathbf{s}_{t}, \mathbf{v}_{t}\right)
\end{array}\right) .
\end{aligned}
$$

Using the instantaneous change of variable theorem in Chen et al. (2018a), we obtain the following expression for the evolution of latent density:

$$
\begin{aligned}
& \frac{\partial \log q\left(\mathbf{z}_{t} \mid \mathcal{W}\right)}{\partial t}=-\operatorname{Tr}\left(\frac{d \tilde{\mathbf{f}}_{\mathcal{W}}\left(\mathbf{z}_{t}\right)}{d \mathbf{z}_{t}}\right)=-\operatorname{Tr}\left(\begin{array}{cc}
\frac{\partial \mathbf{v}_{t}}{\partial \mathbf{s}_{t}} & \frac{\partial \mathbf{v}_{t}}{\partial \mathbf{v}_{t}} \\
\frac{\partial \mathbf{f}_{\mathcal{W}}\left(\mathbf{s}_{t}, \mathbf{v}_{t}\right)}{\partial \mathbf{s}_{t}} & \frac{\partial \mathbf{f}_{\mathcal{W}}\left(\mathbf{s}_{t}, \mathbf{v}_{t}\right)}{\partial \mathbf{v}_{t}}
\end{array}\right) \\
& =-\operatorname{Tr}\left(\frac{\partial \mathbf{f}_{\mathcal{W}}\left(\mathbf{s}_{t}, \mathbf{v}_{t}\right)}{\partial \mathbf{v}_{t}}\right),
\end{aligned}
$$




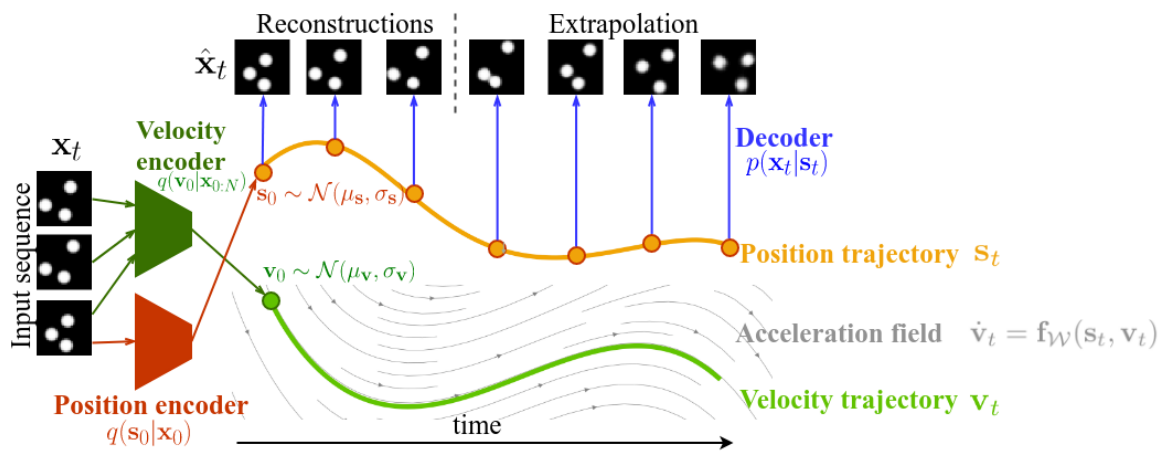

Figure 4.1. A schematic illustration of $\mathrm{ODE}^{2} \mathrm{VAE}$ model taken from Publication III. Position encoder $\left(\mu_{\mathrm{s}}, \sigma_{\mathrm{s}}\right)$ maps the first point $\mathbf{x}_{0}$ in a high-dimensional data sequence into a distribution of the initial position $\mathbf{s}_{0}$ in a latent space. Velocity encoder $\left(\mu_{\mathbf{v}}, \sigma_{\mathrm{v}}\right)$ maps the first $m$ data points $\mathbf{x}_{0: m}$ into an initial velocity distribution $q\left(\mathbf{v}_{0} \mid \mathbf{x}_{0: m}\right)$. Probabilistic latent dynamics are implemented by a second order ODE model $\tilde{\mathbf{f}}_{\mathcal{W}}$ parameterised by a Bayesian neural network $(\mathcal{W})$. Data points in the original data domain are reconstructed by a decoder.

which results in the log densities over time

$$
\log q\left(\mathbf{z}_{N} \mid \mathbf{z}_{0}, \mathcal{W}\right)=\log q\left(\mathbf{z}_{0} \mid \mathcal{W}\right)-\int_{0}^{t_{N}} \operatorname{Tr}\left(\frac{\partial \mathbf{f}_{\mathcal{W}}\left(\mathbf{s}_{t}, \mathbf{v}_{t}\right)}{\partial \mathbf{v}_{t}}\right) d t .
$$

The model components are visualized in Figure 4.1. Note that we set $T=m=3$ in this illustration. The first three decoded frames match the input sequence while the following four frames are unseen.

\subsubsection{Evidence Lower Bound}

In this section, we derive the ELBO and show how the loss term can be augmented with additional terms our model benefits from. For brevity, we denote $X=\mathbf{x}_{0: T}$ and $Z=\mathbf{z}_{0: T}$, and derive the evidence lower bound as follows (Blei et al., 2016):

$$
\begin{aligned}
& \log p(X) \geq \underbrace{-\operatorname{KL}[q(\mathcal{W}, Z \mid X) \| p(\mathcal{W}, Z)]+\mathbb{E}_{q(\mathcal{W}, Z \mid X)}[\log p(X \mid \mathcal{W}, Z)]}_{\text {ELBO }} \\
& =-\mathbb{E}_{q(\mathcal{W}, Z \mid X)}\left[\log \frac{q(\mathcal{W}) q(Z \mid \mathcal{W}, X)}{p(\mathcal{W}) p(Z)}\right]+\mathbb{E}_{q(\mathcal{W}, Z \mid X)}[\log p(X \mid \mathcal{W}, Z)] \\
& =-\mathrm{KL}[q(\mathcal{W}) \| p(\mathcal{W})]+\mathbb{E}_{q(\mathcal{W}, Z \mid X)}\left[-\log \frac{q(Z \mid \mathcal{W}, X)}{p(Z)}+\log p(X \mid \mathcal{W}, Z)\right] \\
& =-\underbrace{\mathrm{KL}[q(\mathcal{W}) \| p(\mathcal{W})]}_{\text {ODE regularization }}+\underbrace{\mathbb{E}_{q \mathrm{enc}\left(\mathbf{z}_{0} \mid X\right)}\left[-\log \frac{q_{\mathrm{enc}}\left(\mathbf{z}_{0} \mid X\right)}{p\left(\mathbf{z}_{0}\right)}+\log p\left(\mathbf{x}_{0} \mid \mathbf{z}_{0}\right)\right]}_{\text {VAE loss }}
\end{aligned}
$$




$$
\begin{aligned}
& +\underbrace{\sum_{i=1}^{T} \mathbb{E}_{q_{\text {enc }}\left(\mathbf{z}_{0} \mid X\right)} \mathbb{E}_{q(\mathcal{W})}\left[\mathbb{E}_{q_{\text {ode }}\left(\mathbf{z}_{i} \mid \mathbf{z}_{0}, \mathcal{W}\right)}\left[-\log \frac{q_{\text {ode }}\left(\mathbf{z}_{i} \mid \mathcal{W}, \mathbf{z}_{0}\right)}{p\left(\mathbf{z}_{i}\right)}\right]\right]}_{\text {dynamic regularization }} \\
& +\underbrace{\sum_{i=1}^{T} \mathbb{E}_{q_{\text {enc }}\left(\mathbf{z}_{0} \mid X\right)} \mathbb{E}_{q(\mathcal{W})}\left[\mathbb{E}_{q_{\text {ode }}\left(\mathbf{z}_{i} \mid \mathcal{W}, \mathbf{z}_{0}\right)}\left[\log p\left(\mathbf{x}_{i} \mid \mathbf{z}_{i}\right)\right]\right]}_{\text {reconstruction }},
\end{aligned}
$$

where we set the prior distribution $p\left(\mathbf{z}_{t}\right)$ to isotropic Gaussian distribution. A state sample $\mathbf{z}_{i}^{(l)} \sim q_{\text {ode }}\left(\mathbf{z}_{i} \mid \mathbf{z}_{0}, \mathcal{W}\right)$ is drawn by first sampling from the approximate posteriors and then forward simulating the dynamics model:

$$
\begin{aligned}
\mathcal{W}^{(l)} & \sim q(\mathcal{W}) \\
\mathbf{z}_{0}^{(l)} & \sim q_{\mathrm{enc}}\left(\mathbf{z}_{0} \mid X\right) \\
\mathbf{z}_{i}^{(l)} & =\mathbf{z}_{0}^{(l)}+\int_{0}^{t_{i}} \tilde{\mathbf{f}}_{\mathcal{W}^{(l)}}\left(\mathbf{z}_{\tau}^{(l)}\right) d \tau .
\end{aligned}
$$

The first term in ELBO (4.4) regularizes the dynamics model, and the second term is identical to original VAE bound. The third term penalizes complex latent state distributions while the last term is responsible for mapping the states into observations. We maximize the ELBO with respect to dynamics, encoder and decoder parameters. We compute the ODE regularization term in closed form while the other expectations are approximated by Monte Carlo sampling.

Penalized loss A well-known pitfall of VAE models is that optimizing the ELBO objective does not necessarily result in accurate inference (Alemi et al., 2018). Borrowing the ideas from Higgins et al. (2017), we weight the $\mathrm{KL}[q(\mathcal{W}) \| p(\mathcal{W})]$ term resulting from the $\mathrm{BNN}$ with a constant factor $\beta$. We choose to fix $\beta$ to the ratio between the latent space dimensionality and number of BNN parameters, $\beta=q /|\mathcal{W}|$, in order to counter-balance the penalties on latent variables $\mathcal{W}$ and $\mathbf{z}_{i}$.

Augmented loss Our variational model utilizes encoders only for obtaining the initial latent distribution. When the input sequences are long, encoder's contribution to ELBO would be much smaller compared to dynamic regularization and reconstruction terms, which are summations over time. Similarly, near-fixed initial distributions $p\left(\mathbf{z}_{0}\right)$ would reduce the effective number of data points the encoder is trained with. To overcome these issues, we augment ELBO with a term that matches the encoder distribution and the distribution induced by the ODE flow, leading to the 
following penalized optimization objective:

$$
\begin{aligned}
& \mathcal{L}_{\mathrm{ODE}^{2} \mathrm{VAE}}=- \beta \mathrm{KL}[q(\mathcal{W}) \| p(\mathcal{W})]+ \\
& \mathbb{E}_{q(\mathcal{W}, Z \mid X)}\left[-\log \frac{q(Z \mid \mathcal{W}, X)}{p(Z)}+\log p(X \mid \mathcal{W}, Z)\right] \\
&-\gamma \mathbb{E}_{q(\mathcal{W})}\left[\operatorname{KL}\left[q_{\text {ode }}(Z \mid X) \| q_{\text {enc }}(Z \mid \mathcal{W}, X)\right]\right] .
\end{aligned}
$$

We choose the constant $\gamma$ by cross-validation. In practice, we found out that an annealing scheme in which $\gamma$ is gradually increased helps optimization, which is also used in (Karl et al., 2016; Rezende and Mohamed, 2015).

Gradient computation Being an alternative to forward sensitivities, adjoint method (2.14-2.15) efficiently computes the loss gradients using Jacobian-vector products. In $\mathrm{ODE}^{2} \mathrm{VAE}$, we utilize the torchdiffeq package that implements adjoints method (Chen et al., 2018b).

\subsection{Experiments}

The model is evaluated on three different datasets: Motion capture, rotating MNIST (Casale et al., 2018) and bouncing balls (Sutskever et al., 2009).

- Motion capture datasets: In addition to the dataset described in Section 3.4.3, we consider a second dataset consisting of 23 walking sequences of subject 35 (Gan et al., 2015), which is partitioned into 16 training, 3 validation and 4 test sequences.

- Rotating MNIST: We repeat the experiment in Casale et al. (2018) by constructing a dataset by rotating the images of handwritten " 3 " digits (16 rotation angles). Overall, the dataset consists of 360 training and 40 validation sequences of $28 \times 28$ frames. We furthermore randomly remove four rotation angles from each sequence to introduce non-uniformity and missing data.

- Bouncing balls: This experiment concerns learning the physical laws of particle collision. For this, we simulate three balls bouncing within a frictionless rectangular box and also colliding with each other using the implementation provided with Sutskever et al. (2009). The dataset consists of video frames of 10000 training and 500 test sequences of length 20 , where each frame is $32 \times 32$ and pixel values vary between 0 and 1 .

In all experiments, our method consistently outperforms the discrete transition baselines. We furthermore highlight different merits of $\mathrm{ODE}^{2} \mathrm{VAE}$ in the subsequent subsections. 
Table 4.2. Average MSE on future frames on mocap datasets

\begin{tabular}{lccr}
\hline \multirow{2}{*}{ Model } & \multicolumn{2}{c}{ Test error } & \\
\cline { 2 - 3 } Gocap-1 & Mocap-2 & Reference \\
\hline VGPM & $126.46 \pm 34$ & N/A & Wang et al. (2006) \\
NPODE & $442.18 \pm 1.92$ & N/A & Damianou et al. (2011) \\
NEURALODE & $87.23 \pm 0.02$ & $22.49 \pm 0.88$ & Chen et al. (2018b) \\
ODE $^{2}$ VAE & $\mathbf{1 5 . 9 9} \pm \mathbf{4 . 1 6}$ & $\mathbf{8 . 0 9} \pm \mathbf{1 . 9 5}$ & Publication III \\
\hline
\end{tabular}

\subsubsection{Robustness against Overfitting}

We compare our model against the methods considered in Section 3.4.3 and vanilla latent neural ODE (Chen et al., 2018b) on mocap datasets. As previously, GPDM, VGPLVM and npODE learn the dynamics in a latent space induced by the PCA mapping. In contrast, a VAE governs the neural ODE latent space similar to our method. The results are presented in Table 4.2. Most strikingly, $\mathrm{ODE}^{2} \mathrm{VAE}$ is superior to other methods (including npODE) on both datasets, justifying our transition from GP-based differential equation modeling to latent neural ODEs. Secondly, while all models achieve smaller test error with more data (second column), the improvement is more drastic for npODE and neural ODE. We conjecture that ML/MAP estimation based parameter inference of these methods leads to overfitting in low-data regimes whereas our variational inference framework is more robust.

\subsubsection{Generalization to Unseen Data Points}

In the rotating MNIST experiment, we aim to illustrate how our model generalizes to interpolate an unseen rotation angle from a sequence of rotating digits. The first rows of Figure $4.2 \mathrm{a}$ and $4.2 \mathrm{~b}$ demonstrate an example training sequence with missing frames and the left-out test angle, respectively. We contrast our method against Gaussian process prior VAE (GPPVAE) (Casale et al., 2018), which replaces the commonly used iid Gaussian prior with a GP and thus performs latent regression. GPPVAE achieves a test mean squared error (MSE) of 0.0309 on the unseen test angle while our $\mathrm{ODE}^{2} \mathrm{VAE}$ reaches a smaller test error (0.0188). For a visual illustration of a rotation sequence and different hand-writing styles $\mathrm{ODE}^{2} \mathrm{VAE}$ generates, please see Figure 4.2. 


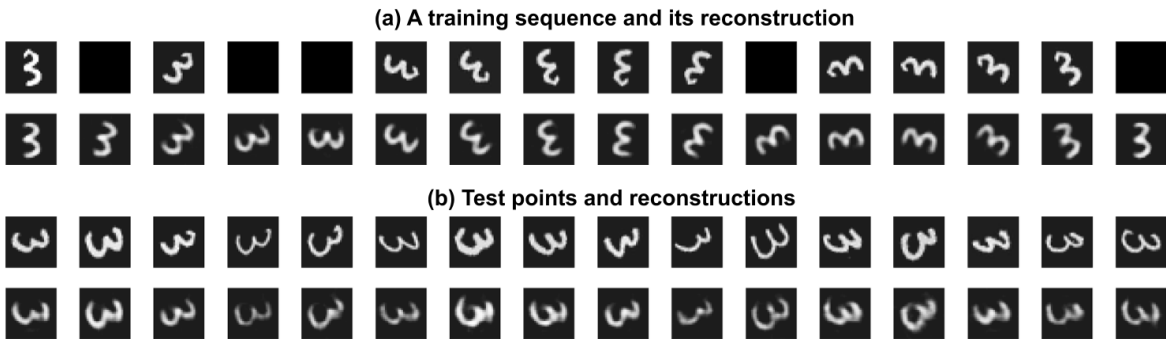

Figure 4.2. Panel (a) shows a training sequence with missing values (first row) and its reconstruction (second row). First row in panel (b) demonstrates test angles from different sequences, i.e., hand-writing styles, and below are model predictions (the figure is taken from Publication III).

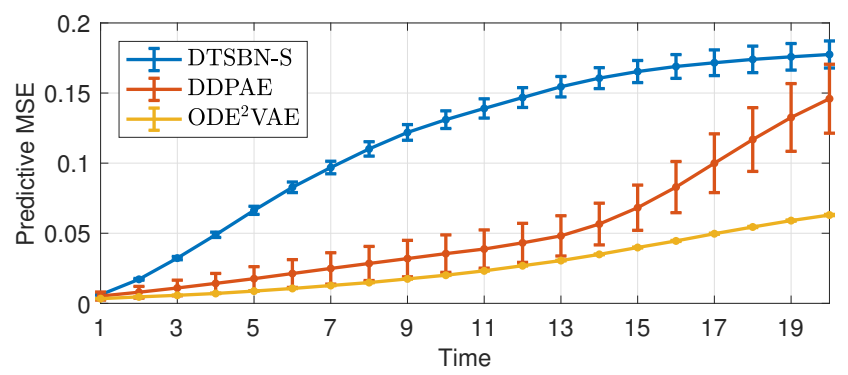

Figure 4.3. Bouncing balls test errors, taken from Publication III.

\subsubsection{The Virtue of Second-Order Modeling}

The competing techniques in our largest-scale bouncing ball experiments are deep temporal sigmoid belief networks (DTSBN-S) (Gan et al., 2015) and decompositional disentangled predictive auto-encoder (DDPAE) (Hsieh et al., 2018). Both methods assume discrete transitions, learn the dynamics in a latent space and performs the same experiment on the respective papers. As shown by the test MSEs in Figure 4.3, our method achieves a much smaller error.

To further investigate $\mathrm{ODE}^{2} \mathrm{VAE}$ modeling choices, we test two variants of our model on the same dataset, where the first variant approximates the dynamics with a NN, and the second variant has the latent space governed by a first-order ODE system (thus named ODE ${ }^{1} \mathrm{VAE}$ ). The test MSEs are given in Table 4.3. As expected, NN dynamics approximation leads to comparable performance since the large number of training sequences prevent the model from overfitting. More interestingly and perhaps not surprisingly, second-order dynamics help with more accurate future predictions, which we attribute to the fact that the underlying physical system is defined in terms of higher order dynamics. 
Table 4.3. Comparison of neural network (NN) and Bayesian neural network (BNN) ODE's with different latent dimensionalities on BOUNCING BALL experiment. Adding 2nd order momentum achieves better performance, while BNN's have a smaller impact.

\begin{tabular}{ccc|ccc}
\hline & \multicolumn{3}{c|}{ Latent dimensions $d$} & \multicolumn{2}{c}{ Test MSE } \\
\cline { 2 - 3 } \cline { 5 - 6 } Model & 1st-order state & 2nd-order momentum & & NN & BNN \\
\hline \multirow{2}{*}{$\mathrm{ODE}^{1} \mathrm{VAE}$} & 25 & - & 45 & 43 \\
& 50 & - & 36 & 35 \\
\hline $\mathrm{ODE}^{2} \mathrm{VAE}$ & 25 & 25 & $\mathbf{2 6}$ & $\mathbf{2 7}$ \\
\hline
\end{tabular}





\section{SDEs for Stochastic Asynchronous Non-convex Optimization}

In the previous chapters, we have described how unknown differential equation systems can be learned using GPs and NNs, which in turn allows accurate long-term predictions. We also demonstrated how ODEs can be coupled with VAEs to handle high dimensional sequences. Thus, the line of research so far has focused only on learning dynamical systems.

We now turn our attention to another problem involving differential equations, namely, stochastic optimization. As briefly touched upon in Section 2.3.2, discretization of SDE systems with carefully crafted drift and diffusion functions leads to stochastic gradient Markov chain Monte Carlo (SGMCMC) algorithms. As an example, we saw that the simplest SGMCMC algorithm, stochastic gradient Langevin dynamics (SGLD), generates samples from a target density by Euler-Maruyama discretization of an SDE system. In an algorithmic sense, SGLD is identical to stochastic gradient descent (SGD), except that it injects a Gaussian noise at each iteration. As shown later, this noise term is the bridge connecting SGMCMC methods (hence SDEs) to optimization, which was also observed in a series of works by Dalalyan (2017); Raginsky et al. (2017); Zhang et al. (2017).

In a related study, Şimşekli et al. (2016a) proposed Hessian approximated MCMC (HAMCMC), an L-BFGS-based SGLD algorithm that significantly speeds up the convergence thanks to the use of second-order Hessian information. Nonetheless, since the standard L-BFGS algorithm requires gradients to be computed on the whole dataset, large scale applications of L-BFGS are quite limited. As a remedy, Berahas et al. (2016) proposed a parallel stochastic L-BFGS algorithm, called multi-batch L-BFGS (mb-LBFGS). The algorithm relies on a synchronized communication between a master node and all workers, each of which operates on a mini-batch of data. This work illustrated that carrying out L-BFGS in a distributed setting introduces further theoretical and practical challenges; however, if these challenges are addressed, stochastic L-BFGS can be powerful in a distributed setting as well.

Although synchronous parallel algorithms have clear advantages over serial optimization algorithms, the computational efficiency of synchronous 


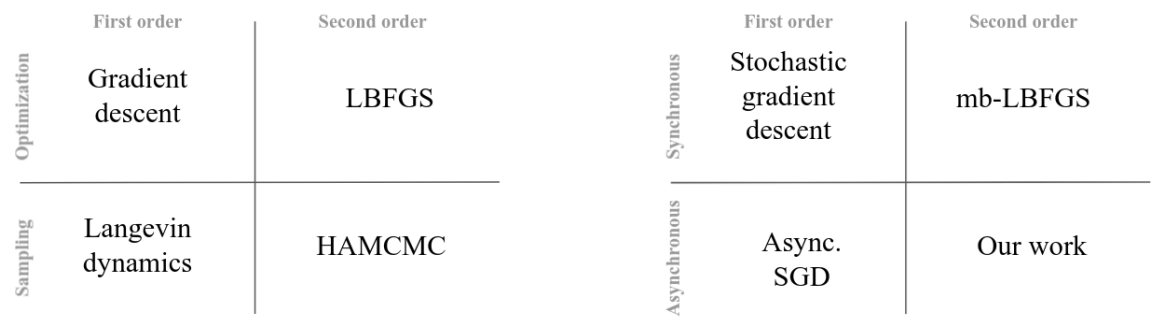

Figure 5.1. A taxonomy of the above-mentioned techniques. Note that all the techniques in the second table are based on stochastic gradients. Building upon the Hessianapproximated SDE dynamics idea of HAMCMC (Şimşekli et al., 2016a) and stochastic L-BFGS idea of mb-L-BFGS (Berahas et al., 2016), we propose a novel asynchronous non-convex optimization method.

algorithms is often limited by the overhead induced by the synchronization and coordination among the worker processes. Inspired by asynchronous parallel stochastic optimization techniques (Agarwal and Duchi, 2011; Lian et al., 2015; Zhang et al., 2015; Zhao and Li, 2016; Zheng et al., 2017), we propose an asynchronous parallel stochastic L-BFGS algorithm for large-scale non-convex optimization problems. Our proposed approach aims at speeding up the synchronous algorithm presented in Berahas et al. (2016) by allowing all the workers work independently from each other and circumvent the inefficiencies caused by synchronization and coordination. We present a taxonomy of the said methods in Figure 5.1.

In the following section, we describe the problem and challenges of stochastic L-BFGS, followed by how tempering bridges the gap between optimization and sampling. Next, we explain our proposed SDE system that generates samples from a target density and show how this SDE can be adapted to a distributed optimization setting. We also repeat the main theoretical finding of our Publication IV for completeness. Section 5.3 details our large scale matrix factorization experiment. Please note that the following sections only aim to convey the ideas, and we refer the reader to Publication IV for the proofs and more detail.

\subsection{Background}

The main problem of interest in this chapter is MAP estimation, which was first presented in the context of numerical optimization (Section 2.3.3). As previously, we are given a set of $N$ data points $\mathcal{D} \equiv\left\{\mathbf{x}_{1}, \ldots, \mathbf{x}_{N}\right\}$ and $\boldsymbol{\theta} \in \mathbb{R}^{P}$ denotes our model parameters. $\boldsymbol{\theta}^{*}$ stands for the optimum of the posterior density $p(\boldsymbol{\theta} \mid \mathcal{D}) \propto \exp (-U(\boldsymbol{\theta}))$, where $U(\boldsymbol{\theta})$ is often called the potential energy function. A stochastic unbiased estimate of the gradient 
$\nabla U(\boldsymbol{\theta})$ is given by

$$
\nabla \tilde{U}(\boldsymbol{\theta})=-\left[\nabla \log p(\boldsymbol{\theta})+\frac{N}{N_{\Omega}} \sum_{i \in \Omega} \nabla \log p\left(\mathbf{x}_{i} \mid \boldsymbol{\theta}\right)\right],
$$

where $\Omega \subset\{1, \ldots, N\}$ denotes a random data subsample that is drawn with replacement and $N_{\Omega}=|\Omega|$ is the cardinality of $\Omega$.

\subsubsection{Stochastic L-BFGS}

The L-BFGS algorithm iteratively applies the following equation in order to find the MAP estimate given in (5.1):

$$
\boldsymbol{\theta}_{n}=\boldsymbol{\theta}_{n-1}-h H_{n} \nabla U\left(\boldsymbol{\theta}_{n-1}\right),
$$

where $n$ denotes the iterations and $h$ is the step size. Here, $H_{n}$ is an approximation to the inverse Hessian at $\boldsymbol{\theta}_{n-1}$ and is computed by using the $M$ past values of the "iterate differences" $\mathbf{s}_{n} \triangleq \boldsymbol{\theta}_{n}-\boldsymbol{\theta}_{n-1}$, and "gradient differences" $\mathbf{y}_{n} \triangleq \nabla U\left(\boldsymbol{\theta}_{n}\right)-\nabla U\left(\boldsymbol{\theta}_{n-1}\right)$ (Nocedal and Wright, 2006). The collection of the iterate and gradient differences is called the $L$-BFGS memory.

In order to achieve computational scalability, stochastic L-BFGS algorithms replace $\nabla U$ with $\nabla \tilde{U}$. This turns out to be problematic since the gradient differences $y_{n}$ would be inconsistent, meaning that the stochastic gradients in different iterations will be computed on different data subsamples, i.e. $\Omega_{n-1} \neq \Omega_{n}$. Furthermore, in the presence of the stochastic gradients, L-BFGS is no longer guaranteed to produce positive definite approximations even in convex problems, therefore more considerations should be taken in order to make sure that $H_{n}$ is positive definite.

\subsubsection{Tempered SGLD}

The connection between SGLD and SGD can be clearly seen by the following tempered version of SGLD:

$$
d \boldsymbol{\theta}_{t}=\nabla U\left(\boldsymbol{\theta}_{t}\right) d t+\sqrt{2 / \beta} W_{t},
$$

where $W_{t}$ denotes the standard Brownian motion in $\mathbb{R}^{P}$. Under mild regularity conditions on $U$, the solution process $\left(\boldsymbol{\theta}_{t}\right)_{t \geq 0}$ attains a unique stationary distribution with a density that is proportional to $\exp (-\beta U(\boldsymbol{\theta}))$ (Roberts and Stramer, 2002). An important property of this distribution is that, as $\beta$ goes to infinity, this density concentrates around the global maximum of $U(\boldsymbol{\theta})$ (Hwang, 1980; Gelfand and Mitter, 1991). Therefore, for large enough $\beta$, a random sample that is drawn for the stationary distribution of $\left(\boldsymbol{\theta}_{t}\right)_{t \geq 0}$ would be close to $\boldsymbol{\theta}^{\star}$.

Building upon this property, Chen et al. (2016) developed an annealed SGMCMC algorithm for non-convex optimization, which was later extended by Ye et al. (2017). Raginsky et al. (2017) and Xu et al. (2018) 
provided finite-time guarantees for SGLD to find an approximate global minimizer that is close to $\boldsymbol{\theta}^{\star}$. Yet, none of these SGMCMC-based techniques describes how to incorporate Hessian information within an asynchronous framework, which we aim to answer in the following.

\subsection{Asynchronous Stochastic L-BFGS}

In this section, we describe our asynchronous L-BFGS-based (tempered) SGMCMC algorithm that aims to provide an approximate optimum that is close to $\boldsymbol{\theta}^{\star}$ by generating samples from a distribution that has a density that is proportional to $\exp (-\beta U(\theta))$. For this, we carefully choose the matrices in (2.21-2.22) so that the resulting system

- utilizes the Hessian information

- allows for tempering to reach the optimum

- becomes computationally feasible and suitable for asynchronous settings

We call the proposed algorithm asynchronous parallel stochastic L-BFGS (as-L-BFGS).

\subsubsection{Designing the Correct SDE}

Similar to Hamiltonian Monte Carlo sampler presented in Section 2.3.2, we consider an augmented setting $X_{t}=\left[\boldsymbol{\theta}_{t}^{\top}, \mathbf{p}_{t}^{\top}\right]^{\top} \in \mathbb{R}^{2 P}$ where $\mathbf{p}_{t} \in \mathbb{R}^{P}$ is called the momentum variable. Given an energy function of the form

$$
\mathcal{E}(X) \triangleq \beta U(\boldsymbol{\theta})+\frac{\beta}{2} \mathbf{p}^{\top} \mathbf{p}
$$

the following SDE has a stationary distribution proportional to $\exp (-\mathcal{E}(X))$ :

$$
\begin{aligned}
d X_{t}=\{ & -(\underbrace{\left[\begin{array}{cc}
0 & 0 \\
0 & \frac{\gamma}{\beta} I
\end{array}\right]}_{\mathbf{D}}+\underbrace{\left[\begin{array}{cc}
0 & -\frac{H_{t}\left(\boldsymbol{\theta}_{t}\right)}{\beta} \\
\frac{H_{t}\left(\boldsymbol{\theta}_{t}\right)}{\beta} & 0
\end{array}\right]}_{\mathbf{Q}_{t}(X)}) \underbrace{\left[\begin{array}{c}
\beta \nabla_{\boldsymbol{\theta}} U\left(\boldsymbol{\theta}_{t}\right) \\
\beta \mathbf{p}_{t}
\end{array}\right]}_{\nabla_{X} \mathcal{E}\left(X_{t}\right)}+\underbrace{\left[\begin{array}{c}
0 \\
\frac{1}{\beta} \Gamma_{t}\left(\boldsymbol{\theta}_{t}\right)
\end{array}\right]}_{\boldsymbol{\Gamma}_{t}\left(X_{t}\right)}] d t \\
& +\sqrt{2 \mathbf{D} d W_{t} .}
\end{aligned}
$$

where $\boldsymbol{\Gamma}_{t}(X)$ was defined in (2.22). Here, $\mathbf{D}$ is positive semi-definite and $\mathbf{Q}$ is skew-symmetric as required in Section 2.3.2. Extending the terms in (5.2) yields the following simplified SDE system:

$$
\begin{aligned}
d \mathbf{p}_{t} & =\left[\frac{1}{\beta} \Gamma_{t}\left(\boldsymbol{\theta}_{t}\right)-H_{t}\left(\boldsymbol{\theta}_{t}\right) \nabla_{\boldsymbol{\theta}} U\left(\boldsymbol{\theta}_{t}\right)-\gamma \mathbf{p}_{t}\right] d t+\sqrt{\frac{2 \gamma}{\beta}} d W_{t} \\
d \boldsymbol{\theta}_{t} & =H_{t}\left(\boldsymbol{\theta}_{t}\right) \mathbf{p}_{t} d t
\end{aligned}
$$


with

$$
\left[\Gamma_{t}(\boldsymbol{\theta})\right]_{i} \triangleq \sum_{j=1}^{P} \frac{\partial\left[H_{t}(\boldsymbol{\theta})\right]_{i j}}{\partial[\boldsymbol{\theta}]_{j}}
$$

where $[v]_{i}$ denotes the $i$ th component of a vector $v$ and similarly $[M]_{i j}$ denotes a single element of a matrix $M$. Here, $H_{t}\left(\boldsymbol{\theta}_{t}\right)$ is an arbitrary symmetric matrix, which we choose to be the L-BFGS matrix. Consequently, (5.3) becomes a dynamical system that uses local geometry information to update the momentum component. Thanks to the convergence property of the SDE, for large enough $\beta$ and $t, \boldsymbol{\theta}_{t}$ would be close to the global optimum $\theta^{\star}$ as desired.

\subsubsection{A Practical Distributed Algorithm}

In order to obtain a practical algorithm, we consider Euler-Maruyama discretization of the SDE system (5.3) with a fixed step size $h$. We also define $\mathbf{u}_{n} \triangleq h \mathbf{p}_{n}, \gamma^{\prime} \triangleq h \gamma, h^{\prime} \triangleq h^{2}$, and discard the term $\Gamma_{n}$ as described in Şimşekli et al. (2016b). These simplifications lead to the following update equations:

$$
\begin{aligned}
& \mathbf{u}_{n+1}=\mathbf{u}_{n}-h^{\prime} H_{n}\left(\boldsymbol{\theta}_{n}\right) \nabla_{\boldsymbol{\theta}} U\left(\boldsymbol{\theta}_{n}\right)-\gamma^{\prime} \mathbf{u}_{n}+\sqrt{2 h^{\prime} \gamma^{\prime} / \beta} Z_{n+1} \\
& \boldsymbol{\theta}_{n+1}=\boldsymbol{\theta}_{n}+H_{n}\left(\boldsymbol{\theta}_{n}\right) \mathbf{u}_{n}
\end{aligned}
$$

\section{Our Distributed Framework}

Next we describe how to adapt above update equations to a distributed setting. We consider a classical asynchronous optimization architecture, which is composed of (i) a master node, (ii) several worker nodes, and a (iii) a data server.

Master node The main task of the master node is to maintain the newest iterate of the algorithm. At each iteration, the master node receives an additive update vector from a worker node, adds this vector to the current iterate in order to obtain the next iterate, and then sends the new iterate to the worker node which has sent the update vector.

Worker node The worker nodes work in a completely asynchronous manner. A worker node receives the iterate from the master node, computes an update vector, and sends the update vector to the master node. However, since the iterate would be possibly modified by another worker node which runs asynchronously in the mean time, the update vector that is sent to the server will thus be computed on an old iterate, which causes both practical and theoretical challenges. Such updates are aptly called "delayed" or "stale". 
Data server The full data is kept in the data server and we assume that all the workers have access to the data server.

In a distributed scenario, the gradient $\nabla U$ in (5.4) needs to be replaced with its stochastic counterpart (5.1). Furthermore, the variables $\boldsymbol{\theta}_{n}$ and $\mathbf{u}_{n}$ are replaced with stale ones $\boldsymbol{\theta}_{n-l_{n}}$ and $\mathbf{p}_{n-l_{n}}$ due to the asynchronous nature of our algorithm. Here, $l_{n} \geq 0$ denotes the "staleness" of a particular update and measures the delay between the current update and the up-todate iterate that is stored in the master node. We assume that the delays are bounded, i.e. $\max _{n} l_{n} \leq l_{\max }<\infty$.

\section{Update Equations}

Master updates The proposed algorithm iteratively applies the following update equations in the master node:

$$
\mathbf{u}_{n+1}=\mathbf{u}_{n}+\Delta \mathbf{u}_{n+1}, \quad \boldsymbol{\theta}_{n+1}=\boldsymbol{\theta}_{n}+\Delta \boldsymbol{\theta}_{n+1},
$$

where $n$ is the iteration index, and $\Delta \mathbf{u}_{n+1}$ and $\Delta \boldsymbol{\theta}_{n+1}$ are the update vectors that are computed by the worker nodes (see below).

Worker updates A worker node runs the following equations in order to compute the update vectors:

$$
\begin{aligned}
& \Delta \mathbf{u}_{n+1} \triangleq-h^{\prime} H_{n+1}\left(\boldsymbol{\theta}_{n-l_{n}}\right) \nabla \tilde{U}_{n+1}\left(\boldsymbol{\theta}_{n-l_{n}}\right)-\gamma^{\prime} \mathbf{u}_{n-l_{n}}+\sqrt{2 h^{\prime} \gamma^{\prime} / \beta} Z_{n+1} \\
& \Delta \boldsymbol{\theta}_{n+1} \triangleq H_{n+1}\left(\boldsymbol{\theta}_{n-l_{n}}\right) \mathbf{u}_{n-l_{n}},
\end{aligned}
$$

where $\nabla \tilde{U}_{n+1}$ denotes the stochastic gradient computed at iteration $n$.

Handling stochastic L-BFGS The master node in mb-L-BFGS algorithm stores a central L-BFGS memory (i.e. the collection of the gradient and iterate differences), and delivers the stored information to the workers in synchronously. However, we let each worker have their own local L-BFGS memories since the master node would not be able to keep track of the gradient and iterate differences due to asynchronousy. In our strategy, each worker updates its own L-BFGS memory right after sending the update vector to the master node.

\subsubsection{Main Theoretical Result}

For completeness, we repeat the main theoretical result of the paper (Theorem 1 in Publication IV).

Theorem 1. Assuming all the conditions presented in Publication IV hold, 
the ergodic error of the proposed algorithm is bounded as follows:

$$
\begin{aligned}
\left|\mathbb{E}\left[\hat{U}_{N}-U^{\star}\right]\right| & =\mathcal{O}\left(\frac{1}{N h}+\max \left(1, l_{\text {max }}\right) h+\frac{1}{\beta}\right) \\
\hat{U}_{N} & \triangleq \frac{1}{N} \sum_{n=1}^{N} U\left(\boldsymbol{\theta}_{n}\right) \\
U^{\star} & \triangleq U\left(\boldsymbol{\theta}^{\star}\right)
\end{aligned}
$$

Theorem 1 provides a non-asymptotic guarantee for convergence to a point that is close to the global optimizer $\boldsymbol{\theta}^{\star}$ even when $U$ is non-convex. The suggested bound on the optimal rate of convergence is in line with the rate of non-convex asynchronous gradient descent algorithm (Lian et al., 2015). Finally, as expected, the error grows linearly with the staleness of the updates $l_{\max }$.

The temperature parameter $\beta$ As shown in the above theorem, the convergence bound is inversely proportional to $\beta$. Hence, we propose to set $\beta$ to large values to speed up the convergence. Note that the inverse of $\beta$ appears as a multiplicative factor of $\Gamma_{n}$, a term that we discarded (see (5.3) again). Consequently, high values of $\beta$ help us diminish the error caused by our negligence, as well. In the following experiment, we set $\beta=10^{3}$ by cross-validation.

\subsection{Large-scale Matrix Factorization Experiment}

The performance of asynchronous stochastic gradient methods has been evaluated in several studies, where the advantages and limitations have been illustrated in various scenarios (Dean et al., 2012; Zhang et al., 2015; Zheng et al., 2017). In this study, in order to illustrate the advantages of asynchrony, we compare as-L-BFGS with mb-L-BFGS Berahas et al. (2016); and in order to illustrate the advantages that are brought by using higherorder geometric information, we compare as-L-BFGS to asynchronous SGD (a-SGD) Lian et al. (2015). We also explore the speedup behavior of as-L-BFGS for increasing the number of workers $W$.

Experiment setup We showcase our method on a large-scale matrix factorization problem (Gemulla et al., 2011), where the goal is to obtain the MAP solution of the following probabilistic generative model:

$$
\begin{aligned}
F_{r k} & \sim \mathcal{N}(0,1) \\
G_{k s} & \sim \mathcal{N}(0,1) \\
Y_{r s} \mid F, G & \sim \mathcal{N}\left(\sum_{k} F_{r k} G_{k s}, 1\right)
\end{aligned}
$$


Table 5.1. A summary of the datasets.

\begin{tabular}{llll}
\hline & \# NONZEROS $(N)$ & \# MOVies $(R)$ & \# USERS $(S)$ \\
\hline ML-1M & 1 million & 3883 & 6040 \\
ML-10M & 10 million & 10681 & 71567 \\
ML-20M & 20 million & 27278 & 138493 \\
\hline
\end{tabular}

Here, $Y \in \mathbb{R}^{R \times S}$ is the data matrix, and $F \in \mathbb{R}^{R \times K}$ and $G \in \mathbb{R}^{K \times S}$ are the factor matrices to be estimated.

In this context, we evaluate the algorithms on three large-scale movie ratings datasets, namely MovieLens (grouplens.org). The properties of the datasets are given in Table 5.1. We have conducted the experiments on a cluster consisting of more than 500 interconnected computers, each of which is equipped with variable quality CPUs and memories. All the three algorithms are implemented in $\mathrm{C}++$ by using a low-level message passing protocol for parallelism, namely the OpenMPI library. This code can be used both in a distributed environment or a single computer with multiprocessors.

Ablations First, we investigate the speedup properties of as-L-BFGS. For this, we only consider the ML-1M dataset and run the as-L-BFGS algorithm for different number of workers. Figure 5.2 illustrates the results of this experiment. As we increase the number of workers $W$ from 1 to 10 , we obtain a decent speedup that is close to linear. However, when we set $W=20$ the algorithm becomes unstable since the term $l_{\max }$ in (5.5) dominates the error. Therefore, for $W=20$ we need to decrease the step-size $h$, which requires the algorithm to be run for a longer amount of time in order to achieve the same error as achieved when $W$ was smaller.

Comparisons Figure 5.3 shows the performance of the three algorithms on the MovieLens datasets in terms of the root-mean-squared-error (RMSE), which is a standard metric for recommendation systems, and the norm
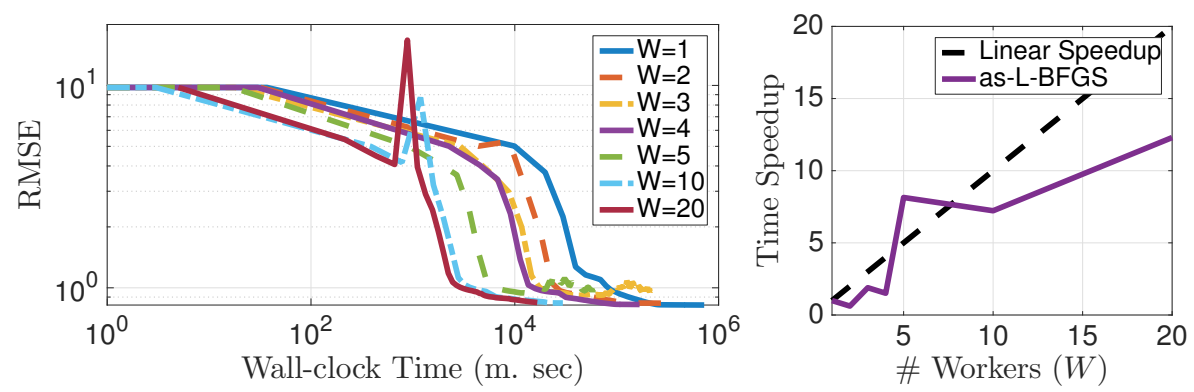

Figure 5.2. The convergence behavior of as-L-BFGS on the ML-1M dataset for increasing number of workers (taken from Publication IV). The "time speedup" is computed as the ratio of the running time with 1 worker to the running time of $W$ workers. 

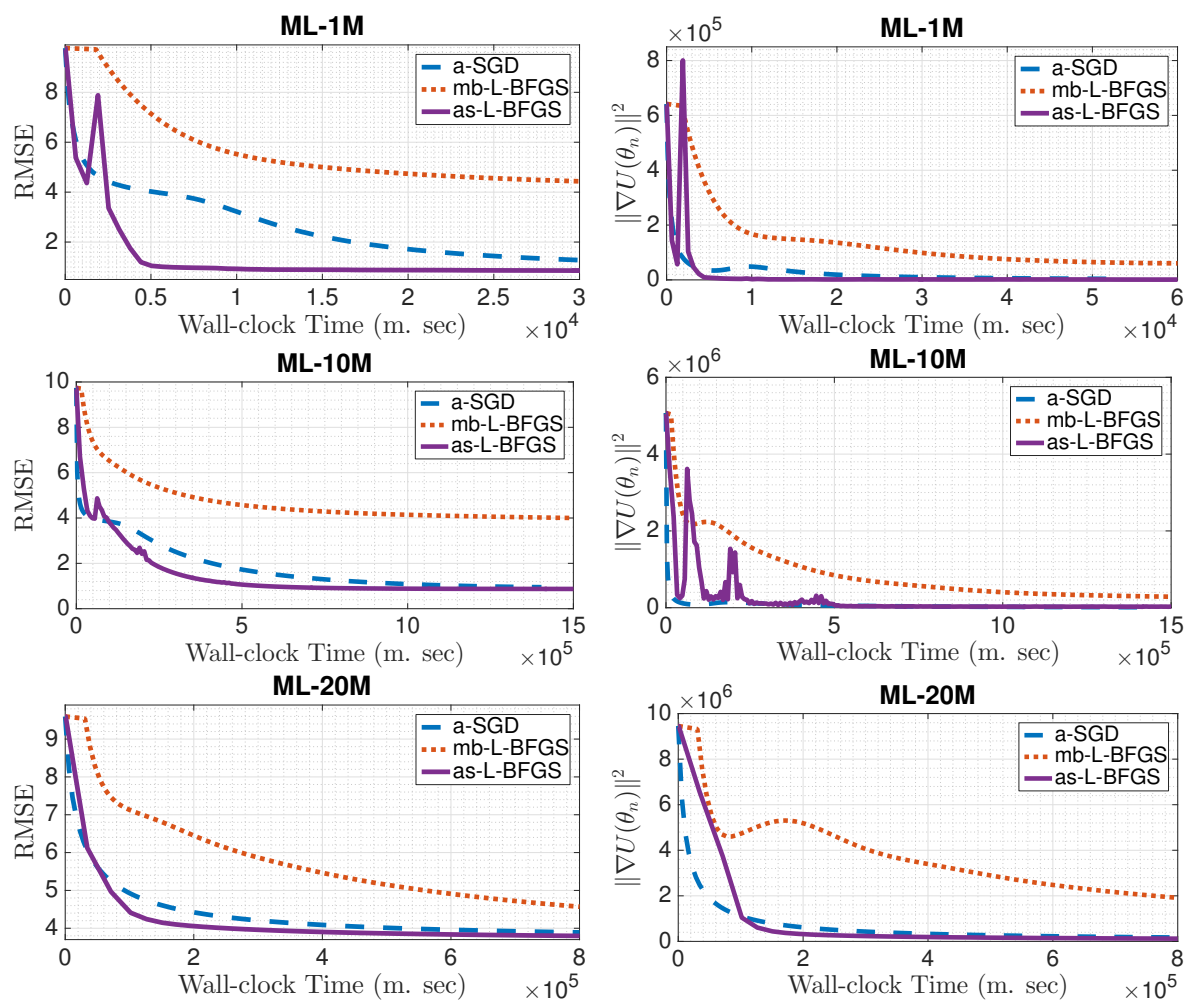

Figure 5.3. The convergence behavior of the algorithms on the MovieLens datasets for $W=10$ (taken from Publication IV).

of the gradients through iterations to measure convergence. In these experiments, we set $K=5$ for all the three datasets and we set the number of workers to $W=10$. The results show that, in all datasets, as-L-BFGS provides a significant speedup over mb-L-BFGS thanks to asynchrony. We can observe that even when the speed of convergence of mb-L-BFGS is comparable to a-SGD and as-L-BFGS (cf. the plots showing the norm of the gradients), the final RMSE yielded by mb-L-BFGS is poorer than the two other methods, which is an indicator that the asynchronous algorithms are able to find a better local minimum. On the other hand, the asynchrony causes more fluctuations in as-L-BFGS when compared to a-SGD.

We also observe that as-L-BFGS provides a slight improvement in the convergence speed when compared to a-SGD. This indicates that a-SGD is able to achieve a comparable convergence speed by taking more steps while as-L-BFGS is computing the matrix-vector products. However, this gap can be made larger by considering a more efficient, yet more sophisticated implementation for L-BFGS computations Chen et al. (2014). 



\section{Continuous-Time Model-Based Reinforcement Learning}

The goal in reinforcement learning $(\mathrm{RL})$ is to build intelligent agents that achieve a goal by learning through experience. In the most general sense, the agent interacts with the outside world, which we refer to as environment. Throughout this chapter, we assume that the environment can be represented by a state vector $\mathbf{s} \in \mathcal{S} \in \mathbb{R}^{n}$ with $\mathcal{S}$ denoting the set of all possible states. The agent interacts with the environment through actions a $\in \mathcal{A} \in \mathbb{R}^{m}$, where $\mathcal{A}$ is a bounded action set. The environment also gives rise to rewards $r \in \mathbb{R}$ to be maximized over a long run (Sutton and Barto, 2018).

The properties of the environment are typically determined by the task of interest. While some of the commonly used benchmark tasks such as chess or Atari games (Schrittwieser et al., 2020) have countably many states and actions, classical RL tasks such as pole balancing (Sutton and Barto, 2018) are naturally defined in continuous state and action spaces. Similarly, the environment may be inherently stochastic or completely deterministic. In certain tasks, the reward is obtained at a fixed frequency while other environments might return the reward once the task terminates (known as sparse rewards). In this work, we focus our attention on continuoustime, state and action problems, where the dynamics are defined by ODEs and the immediate reward is always available. The setting is formally described in Section 6.1.

The particular approach we take to solve RL problems is known as modelbased RL (MBRL). The idea behind MBRL is to infer an approximate dynamics model that can be used to simulate the environment. In turn, the simulations allow the agent to learn a policy that specifies how to act. The vast majority of MBRL methods are based on auto-regressive, discrete-time transition functions that take as input the current state and action and output a new state - thus are infeasible to learn ODEs. To resolve this, we propose to adopt the ideas presented in previous chapters to learn unknown continuous-time dynamical systems involving actions (Section 6.2). We also describe a novel and theoretically consistent continuous-time policy learning algorithm that generalizes its discrete-time counterparts 
Table 6.1. A comparison of existing MBRL methods: PILCO (Deisenroth and Rasmussen, 2011), GP-MPC (Kamthe and Deisenroth, 2018), PETS (Chua et al., 2018), RSSM (Hafner et al., 2018), Dreamer (Hafner et al., 2020), DeLaN (Lutter et al., 2019), SymODEN (Zhong et al., 2020). "Model predictive control" and "proportional-derivative" are abbreviated as MPC and PD.

\begin{tabular}{llll}
\hline MODEL & DynAmics & APPROXimation & CONTROL/RL \\
\hline PILCO & Discrete & GP & Policy \\
GP-MPC & Discrete & GP & MPC \\
PETS & Discrete & Ensembles & MPC \\
RSSM & Discrete & RNN & MPC \\
Dreamer & Discrete & RNN & Policy \\
DeLaN & Lagrangian & NN & PD-controller \\
SymODEN & Hamiltonian & NN & Energy shaping \\
Publication V & Continuous & Ensembles & Policy \\
\hline
\end{tabular}

(Section 6.3). A detailed description of existing approaches, which also highlight the novel aspects of our method, is presented in Section 6.4. Please see Table 6.1 for a summary of the most recent MBRL approaches. Ever-increasing number of RL methods are typically benchmarked on simulation frameworks such as OpenAI Gym (Brockman et al., 2016) and DeepMind Control Suite (Tassa et al., 2018). Both frameworks by-pass the need to contact with the physical world thanks to the MuJoCo physics engine (Todorov et al., 2012). MuJoCo represents multi-joint dynamics in generalized coordinates and performs numerical integration to simulate real-world systems. Consequently, the accuracy of the engine is inherently restricted by the underlying numerical approaches. This often overlooked property of MuJoCo turns out to be highly crucial from a continuous-time modeling standpoint. We provide experimental evidence demonstrating the inaccuracies of existing benchmarks, and present a new framework in Section 6.5. We conclude by demonstrating our method on three different RL problems and show its robustness to both noisy and irregular data, which pose major challenges to conventional discrete-time approaches.

\subsection{Problem Formulation}

A continuous-time and state RL problem is defined by an ODE (Doya, 1996):

$$
\dot{\mathbf{s}}(t)=\mathbf{f}(\mathbf{s}(t), \mathbf{a}(t)),
$$

which was first given in (2.4). The state $\mathbf{s}(t)$ at real-valued time $t \in \mathbb{R}_{+}$ depends on the initial state $\mathbf{s}_{0}$ as well as the infinitesimal sequence of past 
actions $\mathbf{a}[0, t)$, and can be solved with

$$
\mathbf{s}(t)=\mathbf{s}_{0}+\int_{0}^{t} \mathbf{f}\left(\mathbf{s}_{\tau}, \mathbf{a}_{\tau}\right) d \tau
$$

where $\tau \in \mathbb{R}_{+}$is an auxiliary time variable. Our goal is to learn a policy function parameterized by $\phi$ that maps states into actions

$$
\mathbf{a}_{t}=\boldsymbol{\pi}_{\phi}\left(\mathbf{s}_{t}\right)
$$

such that the following discounted infinite horizon reward integral, or value function, is maximized (Sutton and Barto, 2018):

$$
\max _{\phi} V\left(\mathbf{s}_{t}\right)=\int_{t}^{\infty} e^{-\frac{\tau-t}{\eta}} r\left(\mathbf{s}_{\tau}, \mathbf{a}_{\tau}\right) d \tau
$$

Above, the state evolution $\mathbf{s}(t)$ is given by the ODE dynamics (6.2). We also assume a given discount term $\eta>0$ and the instantaneous reward function $r\left(\mathbf{s}_{\tau}, \mathbf{a}_{\tau}\right): \mathbb{R}^{n+m} \rightarrow \mathbb{R}$ which can be differentiated wrt $\mathbf{s}_{\tau}$ and $\mathbf{a}_{\tau}$. Figure 6.1 contrasts the discrete and continuous-time modeling approaches.

Maximizing $V\left(\mathbf{s}_{t}\right)$ wrt policy parameters $\phi$ brings two challenges: (i) computing the derivatives along the path $s[t, \infty)$ governed by the unknown dynamics $\mathbf{f}$ (6.1) and (ii) computing the infinite integral (6.3). We circumvent the first problem by learning neural network dynamics approximation $\mathbf{f}_{\mathcal{W}}$, which allows differentiation via forward sensitivities or adjoints as described in Section 2.2.4. The second problem is tackled by a novel dynamic programming approach to approximate $V\left(\mathbf{s}_{t}\right)$. The following two sections describe our method in detail.

\subsection{Dynamics Learning}

Throughout this section, we assume that the dataset consists of $N$ observed state-action trajectories $\mathcal{D}=\left\{\left(\mathbf{s}_{0: T}^{(n)}, \mathbf{a}_{0: T}^{(n)}\right)\right\}_{n=1}^{N}$ collected from a continuoustime environment we aim to model or, in case of simulated environments, obtained by solving the true ODE system at observation time points $t_{0: T}$. The $i$ 'th observation within a trajectory is denoted by $\mathbf{s}_{i}:=\mathbf{s}\left(t_{i}\right)$ and $\Delta t_{i}=$ $t_{i+1}-t_{i}$ stands for the time until the next observation $\mathbf{s}_{i+1}$. We note that observations may arrive irregularly in time.

We consider deep neural ODE models $\mathbf{f}_{\mathcal{W}}$ to learn the observed trajectories, where $\mathcal{W}$ denotes the network parameters (Chen et al., 2018b). As pointed out in Section 4.2, non-linear neural networks lead to intractable posterior distributions, and we resort to variational inference by introducing an approximate posterior distribution $q(\mathcal{W})$ and evidence lower bound (ELBO):

$$
\log p(\mathcal{D}) \geq \mathbb{E}_{q(\mathcal{W})}[\log p(\mathcal{D} \mid \mathcal{W})]-\mathrm{KL}[q(\mathcal{W}) \| p(\mathcal{W})]
$$



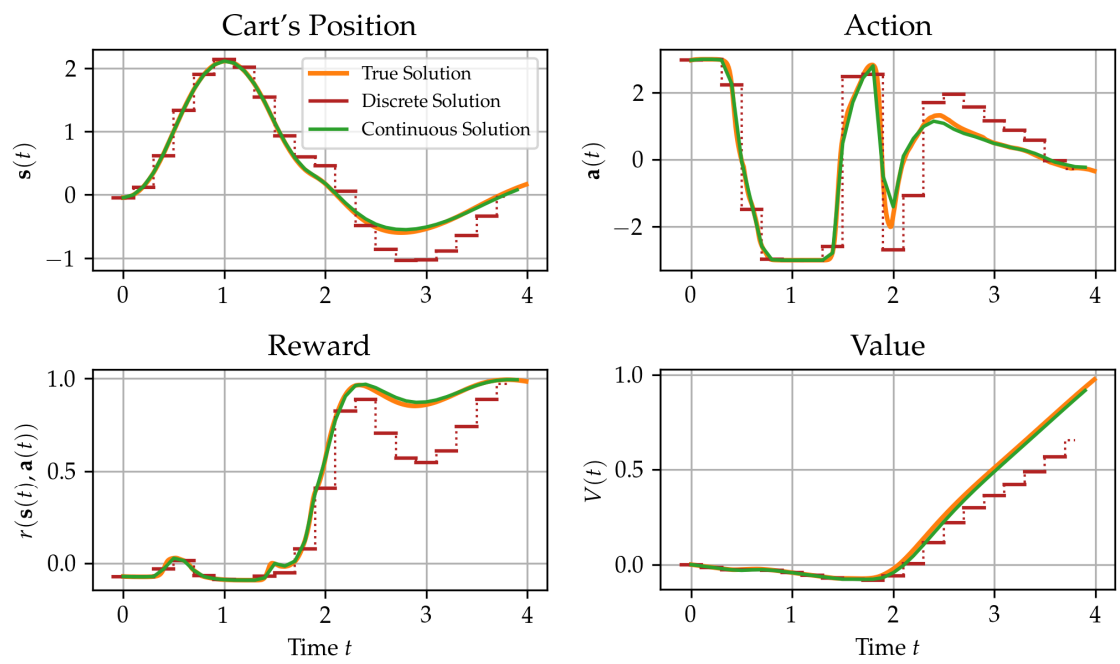

Figure 6.1. We contrast discrete (red) and continuous (green) dynamics approximations against true underlying system (yellow) on the CartPole swing-up problem. While continuous-time solution almost perfectly matches the true solution, the discrete trajectory diverges from it since the discrete approximation error accumulates over time (the figure is reproduced from Publication V).

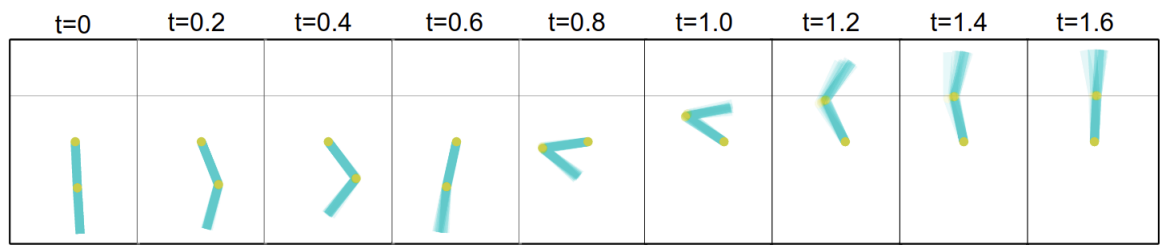

Figure 6.2. We learn a dataset of sequences collected from the Acrobot environment by an ENODE dynamics approximation with 10 ensemble members. We forward simulate all ensemble members starting from the same initial state and applying the same action sequence. Resulting states are visualized at uniform intervals. As shown, the uncertainty grows over time and reaches the peak when the pole is in upright position (the figure is taken from Publication V).

where $p(\mathcal{W})$ is the parameter prior. Note that the likelihood $p(\mathcal{D} \mid \mathcal{W})$ is evaluated only on the state-trajectories, i.e., $p(\mathcal{D} \mid \mathcal{W})=p\left(\mathcal{D}_{\mathbf{s}} \mid \mathcal{D}_{\mathbf{a}}, \mathcal{W}\right)$, where $\mathcal{D}_{\mathbf{s}}=\left\{\mathbf{s}_{0: T}^{(n)}\right\}_{n=1}^{N}$ and $\mathcal{D}_{\mathbf{a}}=\left\{\mathbf{a}_{0: T}^{(n)}\right\}_{n=1}^{N}$. Similar to ODE ${ }^{2}$ VAE, the first expectation above is approximated by Monte Carlo sampling:

$$
\mathbb{E}_{q(\mathcal{W})}[\log p(\mathcal{D} \mid \mathcal{W})] \approx \frac{1}{L} \sum_{l=1}^{L} \sum_{n=1}^{N} \sum_{i=0}^{T} \log \mathcal{N}\left(\mathbf{s}_{i}^{(n)} \mid \hat{\mathbf{s}}_{i}^{(n, l)}, \Sigma\right),
$$

where $\Sigma$ denotes a diagonal matrix of trainable observation noise variances. A trajectory sample $\hat{\mathbf{s}}_{0: T}^{(n, l)}$ is obtained by first sampling from the posterior $\mathcal{W}^{(l)} \sim q(\mathcal{W})$ and then forward simulating the dynamics model $\mathbf{f}_{\mathcal{W}^{(l)}}(\mathbf{s}, \mathbf{a})$.

Ensemble of neural ODEs (ENODEs) A series of MBRL works (Kurutach et al., 2018; Chua et al., 2018; Janner et al., 2019; Pan et al., 2020) showed that deep ensembles typically excel at learning multi-modal posterior 
distributions. In turn, this provides means for epistemic uncertainty modeling, which can be defined as our lack of knowledge about the dynamics in certain regions in the state space due to lack of data. Since epistemic uncertainty handling lies at the heart of successful MBRL applications (Chua et al., 2018), we choose our variational posterior to be a mixture of Dirac densities on the weights, resulting in an ensemble of neural ODEs approximation (ENODEs). As demonstrated in Figure 6.2, each ensemble member indeed leads to slightly different vector fields.

Comparison with our previous models The presented approach is in large part built upon the ideas in Publication I and Publication III. Similar to npODE, we consider a first-order dynamical system (6.5) that is defined in the same (state) space as the observations. As in $\mathrm{ODE}^{2} \mathrm{VAE}$, we opt for a neural network differential function approximation and variational inference for the model parameters. Finally, our approximate posterior distribution is a mixture of Dirac densities since the MAP estimation in Publication I and mean-field approximation in Publication III only provide unimodal approximations to a complicated, multimodal posterior (Izmailov et al., 2021), which prevents us from handling epistemic uncertainty.

Time-continuous actions Any state trajectory is fully determined by the initial state $\mathbf{s}_{0}$ and the infinitesimal actions $\mathbf{a}[0, t)$ applied during integration. Since the numerical integration methods may evaluate the integral at arbitrary time points, the actions must be continuous in time, whereas they are assumed to be known only at observation time points. A simple yet convenient mean to obtain time-continuous actions is interpolating between observed discrete actions. We opt for kernel interpolation with a squared exponential kernel function whose length-scale parameter can be set depending on the smoothness of the action sequence.

\subsection{Continuous-time Actor-Critic}

Actor-critic methods are among the most prominent policy learning techniques (Sutton and Barto, 2018). As implied by its name, actor-critic methods consist of two modules: the actor (policy) that is used to select the actions, and the critic that estimates the value function (6.3). To the best of our knowledge, the actor-critic methods have been presented solely in the context of discrete-transitions previously (Sutton and Barto, 2018). In the following, we describe a novel algorithm that is fully compatible with our continuous-time framework, and the standard discrete-time actor-critic methods (Schulman et al., 2015; Hafner et al., 2020) appear as special cases of our algorithm.

Our main policy learning goal is to maximize the discounted cumulative reward (6.3) when the policy is executed in the real-world. Because the 
true differential function is unknown, we leverage our proxy dynamics $\mathbf{f}_{\mathcal{W}}$ to maximize a surrogate objective:

$$
V\left(\mathbf{s}_{0}\right) \approx \hat{V}\left(\mathbf{s}_{0}\right)=\mathbb{E}_{q(\mathcal{W})}\left[\int_{0}^{\infty} e^{-\frac{\tau}{\eta}} r\left(\hat{\mathbf{s}}_{\tau}, \hat{\mathbf{a}}_{\tau}\right) d \tau\right],
$$

where $\hat{\mathbf{s}}[0, t)$ and $\hat{\mathbf{a}}[0, t)$ are referred to as imagined trajectory and imagined actions ${ }^{1}$ and $\mathbf{s}_{0}$ denotes an initial value. The imagination is controlled by a policy function $\pi_{\phi}(\cdot)$ with parameters $\phi$ :

$$
\begin{aligned}
\hat{\mathbf{s}}(t) & =\mathbf{s}_{0}+\int_{0}^{t} \mathbf{f}_{\mathcal{W}}\left(\hat{\mathbf{s}}_{\tau}, \hat{\mathbf{a}}_{\tau}\right) d \tau \\
\hat{\mathbf{a}}_{t} & =\boldsymbol{\pi}_{\phi}\left(\hat{\mathbf{s}}_{t}\right) .
\end{aligned}
$$

Note that for the dynamics differential $\dot{\mathbf{s}}(t)$ (6.1) to exist, the function $\mathbf{f}_{\mathcal{W}}$ needs to be differentiable over time. When the actions are given by a policy function as in our imagination framework, both $\mathbf{f}_{\mathcal{W}}$ and $\boldsymbol{\pi}_{\phi}$ need to be deterministic. Also, the initial state $\mathbf{s}_{0}$ generally follows a distribution $\mathbf{s}_{0} \sim p\left(\mathbf{s}_{0}\right)$. In such cases, the optimization target becomes $\mathbb{E}_{p\left(\mathbf{s}_{0}\right)}\left[\hat{V}\left(\mathbf{s}_{0}\right)\right]$, which is usually approximated by Monte-Carlo averaging.

\subsubsection{State-Value Approximation (Critic)}

The optimization objective in (6.6) requires computing an infinite integral. To circumvent this problem, we first re-write the value function in a recursive manner:

$$
\hat{V}^{H}\left(\mathbf{s}_{0}\right)=\mathbb{E}_{q(\mathcal{W})}\left[\int_{0}^{H} e^{-\frac{\tau}{\eta}} r\left(\hat{\mathbf{s}}_{\tau}, \hat{\mathbf{a}}_{\tau}\right) d \tau+e^{-\frac{H}{\eta}} \hat{V}\left(\hat{\mathbf{s}}_{H}\right)\right],
$$

where $H$ is known as horizon, $\hat{\mathbf{s}}_{H}:=\hat{\mathbf{s}}(H)$ is the end-state of the integral, and $\hat{V}\left(\hat{\mathbf{s}}_{H}\right)$ is future reward. The first term inside the expectation represents the imagined reward, which can be computed by evaluating the reward function along the trajectory given by (6.7-6.8), whereas the second term is still an infinite integral. Next we introduce the critic, or state-value approximation, that replaces intractable future reward term:

$$
\hat{V}(\mathbf{s}) \approx \nu_{\boldsymbol{\xi}}(\mathbf{s}),
$$

where $\nu_{\boldsymbol{\xi}}$ is a neural network with parameters $\boldsymbol{\xi}$. With a slight abuse of notation, we rewrite the value function as follows:

$$
\hat{V}^{H}\left(\mathbf{s}_{0}\right)=\mathbb{E}_{q(\mathcal{W})}\left[\int_{0}^{H} e^{-\frac{\tau}{\eta}} r\left(\hat{\mathbf{s}}_{\tau}, \hat{\mathbf{a}}_{\tau}\right) d \tau+e^{-\frac{H}{\eta}} \nu_{\boldsymbol{\xi}}\left(\hat{\mathbf{s}}_{H}\right)\right] .
$$

Consequently, our new objective seeks to learn a policy function $\pi_{\phi}$ that maximizes the reward integral over horizon $H$ and ends up at a high-value state.

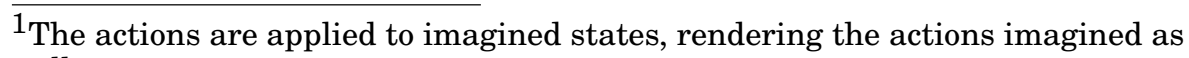
well. 


\subsubsection{Estimating the Actor and Critic}

Actor The actor aims to maximize the value estimates (6.9) for a batch of initial values. In order to learn the optimal policy everywhere, initial values are drawn from the previous experience. More formally, we solve the following maximization problem:

$$
\max _{\phi} \mathbb{E}_{q(\mathbf{s})}\left[\hat{V}^{H}(\mathbf{s})\right], \quad q(\mathbf{s})=\frac{1}{|\mathcal{D}|} \sum_{n, i} \delta\left(\mathbf{s}-\mathbf{s}_{i}^{(n)}\right),
$$

where $|\mathcal{D}|$ denotes the cardinality of the dataset. Observe that both terms in (6.9) depend on the imagined state trajectory, which in turn depends on the actions. Therefore, the gradient of $\hat{V}^{H}(\mathbf{s})$ wrt $\phi$ can easily be computed in auto-differentiation frameworks that support ODE gradients.

Critic Similar to discrete-time frameworks, our method utilizes temporal difference learning to estimate the state-value function. In particular, the value approximation $\nu_{\boldsymbol{\xi}}(\cdot)$ is regressed on the sum of the finite reward integral and future reward given in (6.9). Since the value approximation holds for any horizon length $H$, we propose to average it out:

$$
\min _{\boldsymbol{\xi}} \mathbb{E}_{q(\mathbf{s})}\left[\left(\nu_{\boldsymbol{\xi}}(\mathbf{s})-\mathbb{E}_{q(h)}\left[\hat{V}^{h}(\mathbf{s})\right]\right)^{2}\right], \quad q(h)=\mathcal{U}[0, H]
$$

where the outer expectation is wrt $q(\mathbf{s})$ as shown in (6.10), $\mathcal{U}$ denotes the uniform distribution, and the inner expectation is approximated by MonteCarlo sampling. We use a separate target network that generates the future rewards, an idea that is known to stabilize the critic training (Mnih et al., 2015). We update the target network with a copy of the critic every 100 optimization iterations.

Both actor and critic loss functions require trajectory sampling from the dynamics model. In practice, we forward integrate the dynamics model once and utilize the resulting state-action trajectories to optimize both objectives. The inner expectation in (6.11) can be evaluated using the intermediate states without incurring any additional overhead.

\subsubsection{Connection to Temporal Difference}

Discrete state-value formulation can be retrieved by discretizing the reward integral (6.3) with a predetermined step size $\Delta t_{i} \equiv t_{i}-t_{i-1}$ (Sutton and Barto, 2018):

$$
\bar{V}\left(\mathbf{s}_{t}\right)=\sum_{l=0}^{\infty} \gamma^{l} r\left(\mathbf{s}_{t+l}, \mathbf{a}_{t+l}\right)
$$

where the overbar sign denotes discrete formulation and $\gamma$ is the discount factor. In such a scenario, $\hat{V}^{h}(\mathrm{~s})$ would reduce to so-called $h$-step return: 
$\bar{V}^{h}\left(\mathbf{s}_{t}\right)=\sum_{l=0}^{h-1} \gamma^{l} r_{t+l}+\bar{V}\left(\mathbf{s}_{t+h}\right)$. Marginalizing the horizon would correspond to computing the mean of the $h$-step returns, a commonly used technique to reduce the gradient variance (Schulman et al., 2016). Finally, TD- $h$ learning would be retrieved by simply keeping the horizon fixed at $h$, i.e., with $q(h)$ being a Dirac distribution.

\subsection{Previous Work}

Model-based RL The majority of model-based reinforcement learning methods assume auto-regressive transitions, which effectively learn a distribution over the next state given the current state and action. Unknown transitions are typically approximated by a Gaussian process (Kocijan et al., 2004; Deisenroth and Rasmussen, 2011; Kamthe and Deisenroth, 2018; Levine et al., 2011), multi-layer perceptron (Gal et al., 2016; Depeweg et al., 2017; Nagabandi et al., 2018; Chua et al., 2018) or recurrent neural network (Ha and Schmidhuber, 2018; Hafner et al., 2018, 2020). Such models are typically developed in conjunction with model predictive control (Richards, 2005) used for planning or with a parametric policy.

Continuous-time RL (CTRL) The first model-free CTRL approach, which was proposed by Baird (1993), simultaneously learns value and advantage functions. Bradtke and Duff (1994) developed Q-functions and temporal different learning in the context of semi-Markov decision processes (continuous time, discrete state). Later, Doya (1996) proposed a continuous-time and state version of the temporal difference algorithm. In their seminal paper, Doya (2000) approximated the continuous-time value function by a neural network and derived a greedy policy formulation based on value gradients. Abu-Khalaf and Lewis (2005) proposed a policy-iteration algorithm for the optimal control of continuous-time systems with constrained controllers. An online version of this algorithm was derived in Vrabie and Lewis (2009), which was extended in a series of papers (Luo et al., 2014; Modares et al., 2016; Zhu et al., 2016; Lee and Sutton, 2019). A direct least-squares solution to Hamilton-Jacobi-Bellman equation was studied in Tassa and Erez (2007), requiring no forward integration for value estimation but a bag of tricks to deal with numerical instabilities. In a related work, Mehta and Meyn (2009) proposed an adaptive controller for nonlinear continuous-time systems via a continuous-time analog of the Q-function. Above-mentioned methods are either built upon known dynamics or they are model-free. In either case, the dynamics are assumed to be linear with respect to the action, a premise needed for closed-form optimal policies.

Neural CTRL The neural ODE breakthrough (Chen et al., 2018b) has opened a new research avenue in CTRL. In particular, physics-informed 
continuous-time dynamical systems have gained popularity. For example, Lagrangian mechanics are imposed on the architecture presented in Lutter et al. (2019), which results in near-perfect real-time control of a robot with seven degrees of freedom. Hamiltonian framework, coupled with symplectic solvers, is proven useful for inferring controls from generalized coordinates and momenta (Zhong et al., 2020). Later in Zhong and Leonard (2020), an interpretable latent Lagrangian dynamical system and controller are trained from images. Note that above-mentioned methods describe model-specific recipes for learning controls.

Continuous-time Value Functions In one of the earliest CTRL studies, Baird (1994) showed that the Q-function that appears in Q-learning collapses to the value function in continuous-time, which prevents the use of off-the-shelf discrete-time policy learning methods. Continuous-time counterpart of the Q-function proposed by Baird (1994) and later extended by Tallec et al. (2019) is known as advantage updating. Doya (2000) considered the Euler discretization of the infinite value integral, which leads to an update equation that coincides with the discrete temporal difference update. Later, Tassa and Erez (2007) described an iterative least squares method that directly minimizes Hamiltonian-Jacobi-Bellman (HJB) estimation error, where HJB famously gives the necessary and sufficient condition for optimality.

\subsection{A New CTRL Evaluation Environment}

We experimentally evaluate our model on three CTRL benchmark environments: Pendulum, CartPole and Acrobot, which are already implemented in standard RL frameworks such as OpenAI Gym (Brockman et al., 2016) and DeepMind Control Suite (Tassa et al., 2018). Existing implementations either perform discrete transitions or inaccurate numerical integration routines, such as Euler's and Runge-Kutta's methods with too big discretization step sizes. Thus, we implement a new RL framework which takes as input a policy function $\pi(\cdot)$ and observation time points, performs numerically-stable forward integration, and returns the state trajectory at corresponding time points:

$$
\mathbf{s}(T)=\mathbf{s}_{0}+\int_{0}^{T} \mathbf{f}\left(\mathbf{s}_{\tau}, \boldsymbol{\pi}\left(\mathbf{s}_{\tau}\right)\right) d \tau .
$$

Note that the environment-specific initial state $\mathbf{s}_{0}$ and the true (hidden) time differential $\mathbf{f}(\mathbf{s}, \mathbf{a})$ are specified in the supplementary material of Publication V.

Next, we ask two questions in relation with the simulation environment and numerical integration: (i) which numerical ODE solver one should use, (ii) to what extent our continuous framework differ from its discrete 


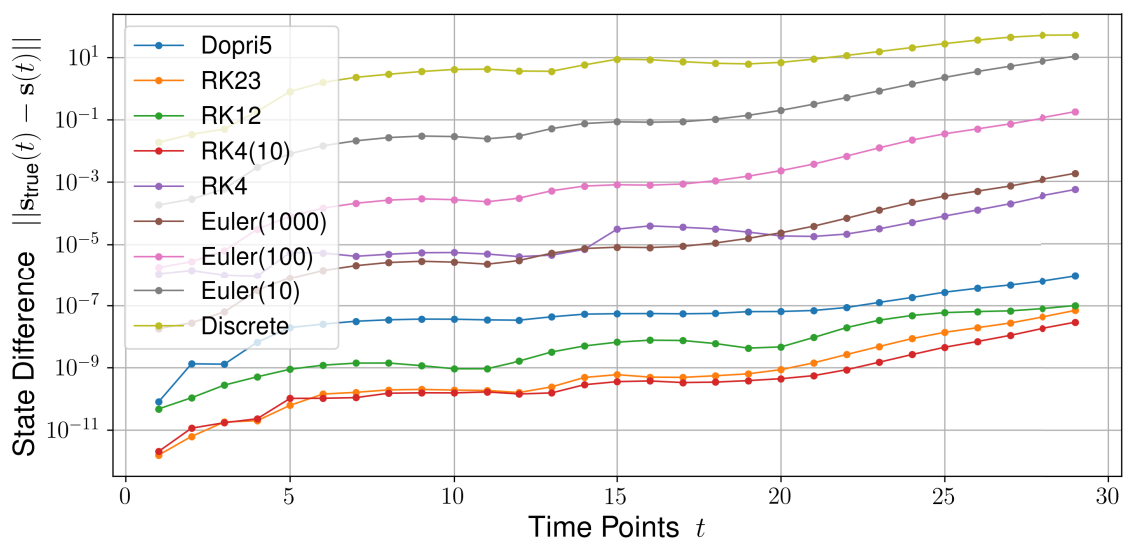

Figure 6.3. Error estimates of different numerical integration methods plotted against integration time (the figure is taken from Publication V).

counterparts? To answer, we have built a simple experiment on CartPole environment where several ODE solvers are compared: three adaptive step solvers (dopri5 (RK45), RK23 and RK12), five fixed step solvers (RK4 with 1/10 intermediate steps, and Euler with 10/100/1000 intermediate steps), as well as discrete transitions. Due to the lack of a closed-form ODE solution, true ODE solutions are obtained by Runge-Kutta 7(8) solver, the numerical integrator which achieves the smallest local error to the best of our knowledge (Prince and Dormand, 1981). Each ODE solver takes as input the same set of initial values and policy functions.

Figure 6.3 demonstrates the distance between the true state solutions and those given by different ODE solvers. The most striking observation is that discrete transitions of the form $\mathbf{s}_{t+1}-\mathbf{s}_{t}=h \cdot \mathbf{f}\left(\mathbf{s}_{t}, \mathbf{a}_{t}\right)$ are highly erroneous. Moreover, adaptive solvers as well as fixed-step solvers with sufficiently many intermediate steps attain practically zero error. Unsurprisingly, approximate state solutions deteriorate over time since the error accumulates. In our experiments, we use RK78 to mimic the interactions with the real world, and dopri5 to forward simulate model dynamics.

\subsection{Experimental Results}

In all three environments, our goal is to bring the pole from downright to upright position (also at the origin in CartPole task). We define the differentiable reward functions as follows:

$$
r(\mathbf{s}, \mathbf{a})=\exp \left(\left\|\mathbf{s}-\mathbf{s}_{\text {goal }}\right\|_{2}\right)-\lambda\|\mathbf{a}\|_{2},
$$

which favors the states that are close to the goal state and the actions small in magnitude. Environment-specific goal states, constant $\lambda$, and lower/upper bounds of allowed actions can also be found in Publication V. 
Learning procedure To learn optimum policy functions, MBRL techniques typically start with a dataset $\mathcal{D}$ of transitions collected by executing a random policy in the real world (Deisenroth and Rasmussen, 2011; Janner et al., 2019). Afterwards, the following procedure is performed: (i) fitting the dynamics model $\mathbf{f}_{\mathcal{W}}$ to the dataset $\mathcal{D}$, (ii) optimizing the policy $\boldsymbol{\pi}_{\phi}$ using the dynamics model, (iii) collecting new sequences by executing the current policy $\pi_{\phi}$ in the real world, and (iv) stop if the problem is solved, else go to step (i). We refer to each iteration of this procedure as a "round".

Observation times and noise We consider three scenarios in which observation arrival times are (i) constant $\kappa$, (ii) uniformly random within a range $\Delta t \sim \mathcal{U}(0,2 \kappa]$, and (iii) exponentially distributed $\operatorname{Exp}(1 / \kappa)$ with $\kappa$ being the rate parameter. Observe that all three cases have the same mean arrival times $\kappa$. We also add an observation noise with standard deviation $\sigma=0.025$.

Compared methods We compare our model with ensemble neural ODE (ENODE) approximation against the state-of-the-art probabilistic ensembles with trajectory sampling (PETS) by Chua et al. (2018). As pointed out in Section 3.1, auto-regressive methods such as PETS are unable to learn irregularly sampled time sequences. In the following, we propose to parameterize the transition with the time increments as follows:

$$
\mathbf{s}_{i+1}=\mathbf{s}_{i}+\mathbf{f}\left(\mathbf{s}_{i}, \mathbf{a}_{i}, \Delta t_{i}\right)
$$

We rename this new model variant modified PETS (MPETS).

Results The results are presented in Figure 6.4. Each panel demonstrates the performance on a single environment with a fixed mean arrival time $\kappa$. $x$-axes correspond to rounds and $y$-axes illustrate the mean values $V\left(\mathbf{s}_{0}\right)$ obtained by executing policies in the real world. We repeat each experiment 20 times and report the mean rewards and 90/10 percent quantiles. As expected, our continuous-time model is rather robust against arrival frequency while small $\kappa$ values are particularly problematic for MPETS. In another ablation study, we observed that both models perform comparably in noise-free scenarios. Therefore, we conclude that our model is more suitable than its discrete counterparts for noisy datasets, and also provide a fundamentally sound approach to handle irregularly sampled sequences. 

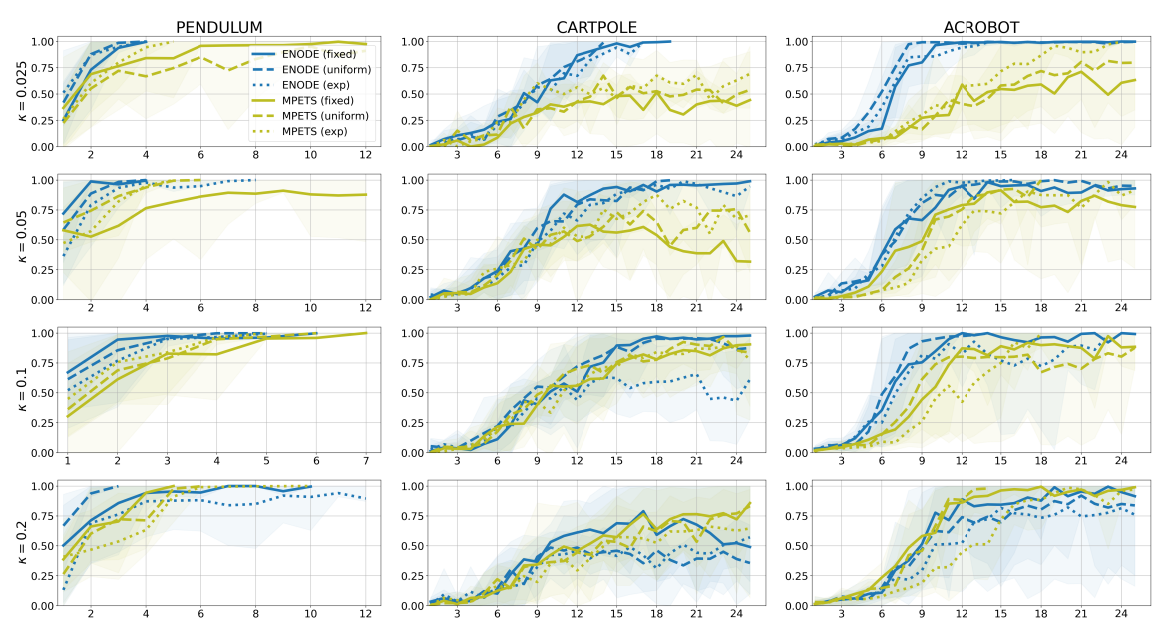

Figure 6.4. ENODE vs. MPETS on noisy environments $(\sigma=0.025)$, taken from Publication V. 


\section{Conclusion and Future Directions}

Thanks to their solid theoretical foundations and decades of previous research, differential equations arise in a wide range of statistical learning problems. This dissertation develops a series of methods in which differential equations played a key role for solving time series prediction, optimization and reinforcement learning problems. Our most significant findings are as follows:

- Parametric ODE and SDE systems can be inferred in a completely data-driven way using black-box function approximations such as GPs and NNs.

- Inferring unknown continuous-time systems with black-box ODEs leads to better predictive performance compared to discrete-time approaches.

- Latent ODE systems can be inferred simultaneously with observation mapping from video sequences.

- An SDE perspective to SGMCMC allows us to conveniently utilize Hessian information and paves the way for an asynchronous and distributed optimization algorithm.

- When coupled with a suitable actor-critic framework, continuous-time dynamical systems provide an alternative avenue for MBRL.

Further research has demonstrated that differential equation formulations have applications in variational inference (Grathwohl et al., 2018), node classification (Poli et al., 2019), generative modeling (Song et al., 2020), speech recognition (Kidger et al., 2020) and so on. Similar to our proposed methods, these techniques utilize differential equations to develop novel strategies for solving distinct statistical learning problems. We conjecture that this very recent marriage between differential equations and machine learning will lead to more exciting and theoretically grounded works and believe that contributions in this dissertation will aid researchers to develop the field further. 
We conclude this dissertation by briefly reminding the proposed ideas, their shortcomings and by suggesting future directions.

GPs for learning unknown differential equations Publication I-II present GP-based estimation schemes for unknown ODEs and SDEs. Our completely black-box approach does not require any prior knowledge about the system dynamics; thus, it presents an alternative paradigm to mechanistic modeling. We experimentally demonstrated that the resulting npODE model is capable of learning the dynamics evolution of human walking sequences for which there is no mechanistic model, and parametric ODE systems without seeing the form of the time differential. Likewise, npSDE model is proven useful for inferring the underlying dynamics of both simulated and real-world sequences such as double-well potential and climate records.

As mentioned in Chapter 3, we compute the MAP estimate of the inducing parameters, which makes the model prone to overfitting. The inference can alternatively be performed by sampling from the posteriors of drift and diffusion functions (Wilson et al., 2020). Similarly, MCMC sampling of the inducing point posterior would lead to trajectory samples instead of a single estimate. Time-varying functions and investigation of different kernel choices reflecting our prior knowledge about the dynamics might also increase model accuracy.

Continuous-time Bayesian modelling for high dimensional sequences In Publication III, we presented ODE ${ }^{2}$ VAE, a neural ODE system governing the dynamics evolution in a latent space induced by a VAE. Unlike our previous approach, we address the uncertainty of the dynamics by computing an approximate posterior over the ODE parameters. Based on the observation that many Newtonian systems are expressed in terms of generalized position and velocity components (Montague, 1995), we explicitly decompose the latent space to account for these components. As shown empirically, the model achieves state-of-the-art performance in long-term forecasting and imputation of high-dimensional image sequences.

Since both the latent mapping and the dynamical system can be arbitrarily expressive, identifying these components becomes a challenging task. The final optimization objective contains a KL term in addition to ELBO, which aims to minimize the distance between the encoder distribution and the distribution induced by the ODE flow. A more principled approach to handle this mismatch might provide new insights. The scope of the experiments was limited to gray-scale images; therefore, demonstrating the model capabilities or extensions to learn dynamics from colored images as well as other high-dimensional data sequences would be a fruitful area for future work. An alternative SDE formulation of the dynamics might further allow the model to learn more complicated scenarios. 
SDEs for stochastic asynchronous non-convex optimization Building upon the recent findings in SGMCMC literature, we propose a stochastic asynchronous non-convex optimization algorithm that converges to a point that is close to the global optimum. Our methodology relies on tempering the SGMCMC target posterior to ensure that the tempered density concentrates around the optimum. We also describe the first asynchronous L-BFGS framework, allowing faster convergence as demonstrated by the experiments.

Despite the non-asymptotic guarantee for convergence to a point that is close to the global optimizer, the experiments show that asynchrony causes a great deal of fluctuations in the loss term. A thorough investigation of how the fluctuations impact the Hessian matrix (and thus optimization) could shed more light on the methodology. Although presented as a nonconvex optimization method, the experiments were performed on convex problems. Further experiments demonstrating the technique on more complicated non-convex problems would be useful to show the merits of the technique.

Continuous-time model-based reinforcement learning Publication V describes a novel continuous-time perspective to MBRL by replacing the discrete-time dynamics approximations with ensemble of neural ODEs, which can learn arbitrarily complicated controlled ODE systems in an uncertainty aware way. We furthermore present the first actor-critic algorithm that operates in continuous time and space and develop a continuoustime RL benchmark that addresses the inaccuracies of standard benchmark frameworks. We experimentally demonstrate that our method is robust to environment changes such as observation noise, temporal sparsity and irregularity.

Although continuous-time formulation achieves a smaller long-term dynamics error, discrete-time baseline still requires a comparable number of trials to solve the problem. Further research should be undertaken to explore how dynamics error impacts the overall performance. Uncertain interpolations between discrete action points could also be tested for better uncertainty handling. Similar to $\mathrm{ODE}^{2} \mathrm{VAE}$, coupling the model with a VAE might allow learning controls from image sequences. 



\section{References}

Martín Abadi, Paul Barham, Jianmin Chen, Zhifeng Chen, Andy Davis, Jeffrey Dean, Matthieu Devin, Sanjay Ghemawat, Geoffrey Irving, Michael Isard, et al. Tensorflow: A system for large-scale machine learning. In Proceedings of the 12th USENIX conference on Operating Systems Design and Implementation, volume 16, pages 265-283, 2016.

Murad Abu-Khalaf and Frank Lewis. Nearly optimal control laws for nonlinear systems with saturating actuators using a neural network HJB approach. Automatica, 41(5):779-791, 2005.

Alekh Agarwal and John C Duchi. Distributed delayed stochastic optimization. In Advances in Neural Information Processing Systems, pages 873-881, 2011.

Tarmo Äijö, Kirsi Granberg, and Harri Lähdesmäki. Sorad: a systems biology approach to predict and modulate dynamic signaling pathway response from phosphoproteome time-course measurements. Bioinformatics, 29(10):12831291, 2013.

Alexander Alemi, Ben Poole, Ian Fischer, Joshua Dillon, Rif A Saurous, and Kevin Murphy. Fixing a broken ELBO. In Proceedings of the 35th International Conference on Machine Learning, pages 159-168. PMLR, 2018.

Christophe Andrieu, Nando De Freitas, Arnaud Doucet, and Michael I Jordan. An introduction to MCMC for machine learning. Machine Learning, 50(1):5-43, 2003.

Kendall Atkinson, Weimin Han, and David E Stewart. Numerical solution of ordinary differential equations, volume 108. John Wiley \& Sons, 2011.

Leemon Baird. Advantage updating. Technical report, Wright Lab, 1993.

Leemon Baird. Reinforcement learning in continuous time: Advantage updating. In IEEE International Conference on Neural Networks, volume 4, pages 24482453, 1994.

David Barber. On solving ordinary differential equations using gaussian processes. arXiv preprint arXiv:1408.3807, 2014.

David Barber, A Taylan Cemgil, and Silvia Chiappa. Bayesian time series models. Cambridge University Press, 2011.

Albert S Berahas, Jorge Nocedal, and Martin Takác. A multi-batch L-BFGS method for machine learning. In Advances in Neural Information Processing Systems, pages 1055-1063, 2016.

Christopher M. Bishop. Pattern Recognition and Machine Learning (Information Science and Statistics). Springer-Verlag, Berlin, Heidelberg, 2006. 
David Blei, Alp Kucukelbir, and Jon McAuliffe. Variational inference: A review for statisticians. Journal of the American Statistical Association, 112:859-877, 2016.

Imke Botha, Robert Kohn, and Christopher Drovandi. Particle methods for stochastic differential equation mixed effects models. Bayesian Analysis, 16(2): 575-609, 2021.

Stephen Boyd and Lieven Vandenberghe. Convex Optimization. Cambridge University Press, USA, 2004.

Phillip Boyle and Marcus Frean. Dependent Gaussian processes. Advances in Neural Information Processing Systems, 17:217-224, 2005.

Andrew M Bradley. PDE-constrained optimization and the adjoint method. Technical report, Technical Report. Stanford University., 2013. URL https: //cs.stanford.edu/ ambrad/adjoint_tutorial.pdf.

Steven Bradtke and Michael Duff. Reinforcement learning methods for continuoustime Markov decision problems. Advances in Neural Information Processing Systems, 7:393-400, 1994.

Greg Brockman, Vicki Cheung, Ludwig Pettersson, Jonas Schneider, John Schulman, Jie Tang, and Wojciech Zaremba. OpenAI gym. arXiv, 2016.

John Charles Butcher and Nicolette Goodwin. Numerical methods for ordinary differential equations, volume 2. Wiley Online Library, 2008.

Ben Calderhead, Mark Girolami, and Neil Lawrence. Accelerating Bayesian inference over nonlinear differential equations with Gaussian processes. Advances in Neural Information Processing Systems, 21:217-224, 2009.

Francesco Paolo Casale, Adrian Dalca, Luca Saglietti, Jennifer Listgarten, and Nicolo Fusi. Gaussian process prior variational autoencoders. In Advances in Neural Information Processing Systems, pages 10369-10380, 2018.

A Taylan Cemgil. A tutorial introduction to monte carlo methods, markov chain monte carlo and particle filtering. Academic Press Library in Signal Processing, $1: 1065-1114,2014$.

Changyou Chen, David Carlson, Zhe Gan, Chunyuan Li, and Lawrence Carin. Bridging the gap between stochastic gradient MCMC and stochastic optimization. In International Conference on Artificial Intelligence and Statistics, 2016.

Changyou Chen, Chunyuan Li, Liqun Chen, Wenlin Wang, Yunchen Pu, and Lawrence Carin. Continuous-time flows for efficient inference and density estimation. Proceedings of the 35th International Conference on Machine Learning, 2018a.

Tian Qi Chen, Yulia Rubanova, Jesse Bettencourt, and David K Duvenaud. Neural ordinary differential equations. In Advances in Neural Information Processing Systems, pages 6571-6583, 2018b.

Weizhu Chen, Zhenghao Wang, and Jingren Zhou. Large-scale L-BFGS using MapReduce. In Advances in Neural Information Processing Systems, pages 1332-1340, 2014.

Kurtland Chua, Roberto Calandra, Rowan McAllister, and Sergey Levine. Deep reinforcement learning in a handful of trials using probabilistic dynamics models. In Advances in Neural Information Processing Systems, pages 4754$4765,2018$. 
Junyoung Chung, Caglar Gulcehre, KyungHyun Cho, and Yoshua Bengio. Empirical evaluation of gated recurrent neural networks on sequence modeling. arXiv preprint arXiv:1412.3555, 2014.

Roberto Confalonieri, Gianni Bellocchi, Simone Bregaglio, Marcello Donatelli, and Marco Acutis. Comparison of sensitivity analysis techniques: a case study with the rice model warm. Ecological Modelling, 221(16):1897-1906, 2010.

Garrison W Cottrell. Learning internal representations from gray-scale images: An example of extensional programming. In Proceedings Ninth Annual Conference of the Cognitive Science Society, Irvine, CA., 1985.

Umut Simşekli, Rolan Badeau, A Taylan Cemgil, and Gael Richard. Stochastic quasi-Newton Langevin Monte Carlo. In Proceedings of the 33st International Conference on Machine Learning, 2016a.

Umut Şimşekli, Roland Badeau, Gale Richard, and A Taylan Cemgil. Stochastic thermodynamic integration: efficient Bayesian model selection via stochastic gradient MCMC. In IEEE Converence on Acoustics, Speech and Signal Processing, 2016b.

George Cybenko. Approximation by superpositions of a sigmoidal function. Mathematics of Control, Signals and Systems, 2(4):303-314, 1989.

Arnak Dalalyan. Further and stronger analogy between sampling and optimization: Langevin Monte Carlo and gradient descent. Proceedings of the 2017 Conference on Learning Theory, 2017.

Andreas Damianou, Michalis K Titsias, and Neil D Lawrence. Variational Gaussian process dynamical systems. In Advances in Neural Information Processing Systems, pages 2510-2518, 2011.

Michiel JL De Hoon, Seiya Imoto, Kazuo Kobayashi, Naotake Ogasawara, and Satoru Miyano. Inferring gene regulatory networks from time-ordered gene expression data of bacillus subtilis using differential equations. In Biocomputing 2003, pages 17-28. World Scientific, 2002.

Jeffrey Dean, Greg Corrado, Rajat. Monga, Kai Chen, Matthieu Devin, Mark Mao, Marc'aurelio Ranzato, Andrew Senior, Paul Tucker, Ke Yang, and Andrew Ng. Large scale distributed deep networks. In Advances in Neural Information Processing Systems, pages 1223-1231, 2012.

Marc Deisenroth and Carl E Rasmussen. PILCO: A model-based and data-efficient approach to policy search. In Proceedings of the 28th International Conference on Machine Learning, pages 465-472, 2011.

Stefan Depeweg, José Miguel Hernández-Lobato, Finale Doshi-Velez, and Steffen Udluft. Learning and policy search in stochastic dynamical systems with Bayesian neural networks. In International Conference on Learning Representations, 2017.

Frank Dondelinger, Maurizio Filippone, Simon Rogers, and Dirk Husmeier. ODE parameter inference using adaptive gradient matching with Gaussian processes. The Journal of Machine Learning Research, 2013.

Georg Dorffner. Neural networks for time series processing. In Neural Network World. Citeseer, 1996.

John R Dormand and Peter J Prince. A family of embedded runge-kutta formulae. Journal of Computational and Applied Mathematics, 6(1):19-26, 1980.

Kenji Doya. Temporal difference learning in continuous time and space. Advances in Neural Information Processing Systems, pages 1073-1079, 1996. 
Kenji Doya. Reinforcement learning in continuous time and space. Neural Computation, 12(1):219-245, 2000.

Simon Duane, Anthony D Kennedy, Brian J Pendleton, and Duncan Roweth. Hybrid monte carlo. Physics letters B, 195(2):216-222, 1987.

James Durbin and Siem Jan Koopman. Time series analysis by state space methods. Oxford university press, 2012.

Stephen P. Ellner, Yodit Seifu, and Rob Smith. Fitting population dynamic models to time-series data by gradient matching. Ecology, 83(8):2256-2270, 2002.

Paul F Evangelista, Mark J Embrechts, and Boleslaw K Szymanski. Taming the curse of dimensionality in kernels and novelty detection. In Applied soft computing technologies: The challenge of complexity, pages 425-438. Springer, 2006.

Erwin Fehlberg. Low-order classical Runge-Kutta formulas with stepsize control and their application to some heat transfer problems, volume 315. National Aeronautics and Space Administration, 1969.

Fabian Fröhlich, Barbara Kaltenbacher, Fabian Theis, and Jan Hasenauer. Scalable parameter estimation for genome-scale biochemical reaction networks. PLOS Computational Biology, 13:1-18, 2017.

Yarin Gal, Rowan McAllister, and Carl Edward Rasmussen. Improving PILCO with Bayesian neural network dynamics models. In ICML Data-Efficient Machine Learning Workshop, page 34, 2016.

Zhe Gan, Chunyuan Li, Ricardo Henao, David E Carlson, and Lawrence Carin. Deep temporal sigmoid belief networks for sequence modeling. In Advances in Neural Information Processing Systems, pages 2467-2475, 2015.

Pei Gao, Antti Honkela, Magnus Rattray, and Neil D Lawrence. Gaussian process modelling of latent chemical species: applications to inferring transcription factor activities. Bioinformatics, 24(16):i70-i75, 2008.

Saul Gelfand and Sanjoy Mitter. Recursive stochastic algorithms for global optimization in $\mathrm{R}^{\wedge} \mathrm{d}$. SIAM Journal on Control and Optimization, 29(5):9991018, 1991.

Rainer Gemulla, Erik Nijkamp, Haas. Peter J., and Yannis Sismanis. Large-scale matrix factorization with distributed stochastic gradient descent. In ACM Special Interest Group on Knowledge Discovery and Data Mining, 2011.

Ian Goodfellow, Yoshua Bengio, and Aaron Courville. Deep Learning. MIT Press, 2016.

Thore Graepel. Solving noisy linear operator equations by Gaussian processes: Application to ordinary and partial differential equations. Proceedings of the 20th International Conference on Machine Learning, 2003.

Will Grathwohl, Ricky TQ Chen, Jesse Betterncourt, Ilya Sutskever, and David Duvenaud. Ffjord: Free-form continuous dynamics for scalable reversible generative models. arXiv preprint arXiv:1810.01367, 2018.

Alex Graves. Practical variational inference for neural networks. In Proceedings of the 24th International Conference on Neural Information Processing Systems, Red Hook, NY, USA, 2011.

David Ha and Jürgen Schmidhuber. World models. arXiv:1803.10122, 2018. 
Danijar Hafner, Timothy Lillicrap, Ian Fischer, Ruben Villegas, David Ha, Honglak Lee, and James Davidson. Learning latent dynamics for planning from pixels. arXiv:1811.04551, 2018.

Danijar Hafner, Timothy Lillicrap, Jimmy Ba, and Mohammad Norouzi. Dream to control: Learning behaviors by latent imagination. In International Conference on Learning Representations, 2020.

Barbara Hammer and Kai Gersmann. A note on the universal approximation capability of support vector machines. Neural Processing Letters, 17(1):43-53, 2003.

Stephen José Hanson and Lorien Y Pratt. Comparing biases for minimal network construction with back-propagation. In Proceedings of the 1st International Conference on Neural Information Processing Systems, pages 177-185, 1988.

Markus Heinonen and Florence d'Alche Buc. Learning nonparametric differential equations with operator-valued kernels and gradient matching. Technical report, Telecom ParisTech, 2014.

Markus Heinonen, Cagatay Yildiz, Henrik Mannerström, Jukka Intosalmi, and Harri Lähdesmäki. Learning unknown ODE models with Gaussian processes. In Proceedings of the 35th International Conference on Machine Learning, volume 80 of Proceedings of Machine Learning Research, pages 1959-1968. PMLR, 2018.

James Henderson and George Michailidis. Network reconstruction using nonparametric additive ode models. PLOS ONE, 2014.

James Hensman, Nicolo Fusi, and Neil Lawrence. Gaussian processes for big data. In Uncertainty in Artificial Intelligence, pages 282-290. AUAI Press, 2013.

Karl Heun. Neue methoden zur approximativen integration der differentialgleichungen einer unabhängigen veränderlichen. Z. Math. Phys, 45:23-38, 1900.

Irina Higgins, Loic Matthey, Arka Pal, Christopher Burgess, Xavier Glorot, Matthew Botvinick, Shakir Mohamed, and Alexander Lerchner. beta-vae: Learning basic visual concepts with a constrained variational framework. In International Conference on Learning Representations, volume 3, 2017.

Sepp Hochreiter and Jürgen Schmidhuber. Long short-term memory. Neural Computation, 9(8):1735-1780, 1997.

Jun-Ting Hsieh, Bingbin Liu, De-An Huang, Li F Fei-Fei, and Juan Carlos Niebles. Learning to decompose and disentangle representations for video prediction. In Advances in Neural Information Processing Systems, pages 517-526, 2018.

Chii-Ruey Hwang. Laplace's method revisited: weak convergence of probability measures. The Annals of Probability, pages 1177-1182, 1980.

Pavel Izmailov, Sharad Vikram, Matthew D Hoffman, and Andrew Gordon Gordon Wilson. What are bayesian neural network posteriors really like? In Proceedings of the 38th International Conference on Machine Learning, volume 139, pages 4629-4640, 2021.

Michael Janner, Justin Fu, Marvin Zhang, and Sergey Levine. When to trust your model: Model-based policy optimization. In Advances in Neural Information Processing Systems, pages 12519-12530, 2019.

Arne Jensen. Lecture notes on difference equations. Department of mathematical science, Aalborg University, Denmark, July, 18, 2011. 
Michael I. Jordan, Zoubin Ghahramani, Tommi S. Jaakkola, and Lawrence K. Saul. An introduction to variational methods for graphical models. Machine Learning, 37(2):183-233, 1999.

Rudolph Kalman. A New Approach to Linear Filtering and Prediction Problems. Journal of Basic Engineering, 82(1):35-45, 1960.

Sanket Kamthe and Marc Deisenroth. Data-efficient reinforcement learning with probabilistic model predictive control. In International Conference on Artificial Intelligence and Statistics, pages 1701-1710. PMLR, 2018.

Maximilian Karl, Maximilian Soelch, Justin Bayer, and Patrick van der Smagt. Deep variational Bayesian filters: Unsupervised learning of state space models from raw data. International Conference on Learning Representations, 2016.

Patrick Kidger, James Morrill, James Foster, and Terry Lyons. Neural controlled differential equations for irregular time series. Advances in Neural Information Processing Systems, 2020.

Diederik P. Kingma and Max Welling. Auto-Encoding Variational Bayesian. In International Conference on Learning Representations, 2014.

Jus Kocijan, Roderick Murray-Smith, Carl Edward Rasmussen, and Agathe Girard. Gaussian process model based predictive control. In Proceedings of the 2004 American Control Conference, volume 3, pages 2214-2219. IEEE, 2004.

Anders Krogh and John Hertz. A simple weight decay can improve generalization. In Advances in Neural Information Processing Systems, volume 4, 1992.

Thanard Kurutach, Ignasi Clavera, Yan Duan, Aviv Tamar, and Pieter Abbeel. Model-ensemble trust-region policy optimization. In International Conference on Learning Representations, 2018.

Malte Kuss and Carl Edward Rasmussen. Assessing approximate inference for binary Gaussian process classification. Journal of Machine Learning Research, 6(Oct):1679-1704, 2005.

Wilhelm Kutta. Beitrag zur naherungsweisen integration totaler differentialgleichungen. Z. Math. Phys., 46:435-453, 1901.

Paul Langevin. Sur la théorie du mouvement Brownien (on the theory of Brownian motion). Comptes-rendus de l'Académie des Sciences (Paris), pages 530-533, 1908.

Neil Lawrence and Aapo Hyvärinen. Probabilistic non-linear principal component analysis with Gaussian process latent variable models. Journal of Machine Learning Research, 6(11), 2005.

Yann LeCun, Bernhard Boser, John S Denker, Donnie Henderson, Richard E Howard, Wayne Hubbard, and Lawrence D Jackel. Backpropagation applied to handwritten zip code recognition. Neural Computation, 1(4):541-551, 1989.

Yann LeCun, Yoshua Bengio, and Geoffrey Hinton. Deep learning. Nature, 521 (7553):436-444, 2015.

Jae Young Lee and Richard S Sutton. Policy iterations for reinforcement learning problems in continuous time and space: Fundamental theory and methods. Automatica, 2019.

Don S Lemons and Anthony Gythiel. Paul Langevin's 1908 paper "on the theory of Brownian motion" [sur la théorie du mouvement Brownien," cr acad. sci.(paris) 146, 530-533 (1908)]. American Journal of Physics, 65(11):1079-1081, 1997. 
Sergey Levine, Zoran Popovic, and Vladlen Koltun. Nonlinear inverse reinforcement learning with Gaussian processes. In Advances in Neural Information Processing Systems, pages 19-27, 2011.

Xiangru Lian, Yijun Huang, Y. Li, and Ji Liu. Asynchronous parallel stochastic gradient for nonconvex optimization. In Advances in Neural Information Processing Systems, pages 2737-2745, 2015.

Seppo Linnainmaa. The representation of the cumulative rounding error of an algorithm as a taylor expansion of the local rounding errors. Master's Thesis (in Finnish), University of Helsinki, pages 6-7, 1970.

Haitao Liu, Yew-Soon Ong, Xiaobo Shen, and Jianfei Cai. When Gaussian process meets big data: A review of scalable gps. IEEE Transactions on Neural Networks and Learning Systems, 31(11):4405-4423, 2020.

Lennart Ljung. Perspectives on system identification. Annual Reviews in Control, 34(1):1-12, 2010.

Biao Luo, Huai-Ning Wu, Tingwen Huang, and Derong Liu. Data-based approximate policy iteration for affine nonlinear continuous-time optimal control design. Automatica, 50(12):3281-3290, 2014.

Michael Lutter, Christian Ritter, and Jan Peters. Deep Lagrangian networks: Using physics as model prior for deep learning. In International Conference on Learning Representations, 2019.

Yi-An Ma, Tianqi Chen, and Emily Fox. A complete recipe for stochastic gradient MCMC. In Advances in Neural Information Processing Systems, pages 28992907, 2015.

Benn Macdonald. Statistical inference for ordinary differential equations using gradient matching. PhD thesis, University of Glasgow, 2017.

Benn Macdonald, Catherine Higham, and Dirk Husmeier. Controversy in mechanistic modelling with Gaussian processes. In Proceedings of the 32nd International Conference on Machine Learning, pages 1539-1547, 2015.

David JC MacKay. A practical Bayesian framework for backpropagation networks. Neural Computation, 4(3):448-472, 1992.

Warren S McCulloch and Walter Pitts. A logical calculus of the ideas immanent in nervous activity. The Bulletin of Mathematical Biophysics, 5(4):115-133, 1943.

Prashant Mehta and Sean Meyn. Q-learning and Pontryagin's minimum principle. In IEEE Conference on Decision and Control, pages 3598-3605, 2009.

Volodymyr Mnih, Koray Kavukcuoglu, David Silver, Andrei A Rusu, Joel Veness, Marc G Bellemare, Alex Graves, Martin Riedmiller, Andreas K Fidjeland, Georg Ostrovski, et al. Human-level control through deep reinforcement learning. Nature, 518(7540):529, 2015.

Hamidreza Modares, Frank L Lewis, and Zhong-Ping Jiang. Optimal outputfeedback control of unknown continuous-time linear systems using off-policy reinforcement learning. IEEE Transactions on Cybernetics, 46(11):2401-2410, 2016.

BW Montague. Basic Hamiltonian mechanics. CERN EUROPEAN ORGANIZATION FOR NUCLEAR RESEARCH-REPORTS-CERN, pages 1-14, 1995.

Kevin P. Murphy. Machine learning : a probabilistic perspective. MIT Press, 2013. 
Anusha Nagabandi, Gregory Kahn, Ronald S Fearing, and Sergey Levine. Neural network dynamics for model-based deep reinforcement learning with model-free fine-tuning. In 2018 IEEE International Conference on Robotics and Automation (ICRA), pages 7559-7566. IEEE, 2018.

Radford m Neal. Bayesian Learning for Neural Networks. PhD thesis, University of Toronto, 1994.

Radford M Neal et al. Mcmc using Hamiltonian dynamics. Handbook of Markov chain Monte Carlo, 2(11):2, 2011.

Jorge Nocedal and Stephen J. Wright. Numerical Optimization: Springer Series in Operations Research and Financial Engineering. Springer, 2006.

Bernt Øksendal. Stochastic Differential Equations: An Introduction with Applications. Springer, 6th edition, 2014.

Feiyang Pan, Jia He, Dandan Tu, and Qing He. Trust the model when it is confident: Masked model-based actor-critic. In Advances in Neural Information Processing Systems, 2020.

Razvan Pascanu, Tomas Mikolov, and Yoshua Bengio. On the difficulty of training recurrent neural networks. In Proceedings of the 30th International Conference on Machine Learning, pages 1310-1318. PMLR, 2013.

Adam Paszke, Sam Gross, Francisco Massa, Adam Lerer, James Bradbury, Gregory Chanan, Trevor Killeen, Zeming Lin, Natalia Gimelshein, Luca Antiga, Alban Desmaison, Andreas Kopf, Edward Yang, Zachary DeVito, Martin Raison, Alykhan Tejani, Sasank Chilamkurthy, Benoit Steiner, Lu Fang, Junjie Bai, and Soumith Chintala. Pytorch: An imperative style, high-performance deep learning library. In Advances in Neural Information Processing Systems, volume $32,2019$.

Kokotovic Petar and James Heller. Direct and adjoint sensitivity equations for parameter optimization. IEEE Trans. on Automatic Control, 12:609-610, 1967.

Michael Poli, Stefano Massaroli, Junyoung Park, Atsushi Yamashita, Hajime Asama, and Jinkyoo Park. Graph neural ordinary differential equations. arXiv preprint arXiv:1911.07532, 2019.

Lev Semenovich Pontryagin. Mathematical theory of optimal processes. Routledge, 2018.

Lev Semenovich Pontryagin, V G Boltyanskii, R V Gamkrelidze, and E F Mishchenko. The mathematical theory of optimal processes. Wiley, New York, NY, 1962.

Lutz Prechelt. Early stopping-but when? In Neural Networks: Tricks of the Trade, This Book is an Outgrowth of a 1996 NIPS Workshop, page 55-69, Berlin, Heidelberg, 1998. Springer-Verlag.

Peter J Prince and John R Dormand. High order embedded runge-kutta formulae. Journal of Computational and Applied Mathematics, 7(1):67-75, 1981.

Joaquin Quiñonero-Candela and Carl Edward Rasmussen. A unifying view of sparse approximate Gaussian process regression. Journal of Machine Learning Research, 6(Dec):1939-1959, 2005.

Christopher Rackauckas, Yingbo Ma, Vaibhav Dixit, Xingjian Guo, Mike Innes, Jarrett Revels, Joakim Nyberg, and Vijay Ivaturi. A comparison of automatic differentiation and continuous sensitivity analysis for derivatives of differential equation solutions. arXiv preprint arXiv:1812.01892, 2018. 
M. Raginsky, A. Rakhlin, and M. Telgarsky. Non-convex learning via stochastic gradient Langevin dynamics: a nonasymptotic analysis. In Proceedings of the 2017 Conference on Learning Theory, volume 65, pages 1674-1703, 2017.

Maziar Raissi, Paris Perdikaris, and George Em Karniadakis. Inferring solutions of differential equations using noisy multi-fidelity data. Journal of Computational Physics, 335:736-746, 2017.

James Ramsay, Giles Hooker, David Campbell, and Jiguo Cao. Parameter estimation for differential equations: a generalized smoothing approach. Journal of the Royal Statistical Society: Series B, 69:741-796, 2007.

Carl E. Rasmussen and Christopher K.I. Williams. Gaussian processes for machine learning. MIT Press, 2006.

Danilo Rezende and Shakir Mohamed. Variational inference with normalizing flows. In Proceedings of the 32nd International Conference on Machine Learning, volume 37, pages 1530-1538. PMLR, 2015.

Danilo Jimenez Rezende, Shakir Mohamed, and Daan Wierstra. Stochastic backpropagation and approximate inference in deep generative models. In Proceedings of the 31st International Conference on Machine Learning, volume 32 of Proceedings of Machine Learning Research, pages 1278-1286. PMLR, 2014.

Arthur George Richards. Robust constrained model predictive control. PhD thesis, Massachusetts Institute of Technology, 2005.

Gareth Roberts and Osnat Stramer. Langevin Diffusions and Metropolis-Hastings Algorithms. Methodology and Computing in Applied Probability, 4(4):337-357, 2002.

James C Robinson. An introduction to ordinary differential equations. Cambridge University Press, 2004.

Frank Rosenblatt. Principles of neurodynamics. perceptrons and the theory of brain mechanisms. Technical report, Cornell Aeronautical Lab Inc Buffalo NY, 1961.

David E Rumelhart, Geoffrey E Hinton, and Ronald J Williams. Learning representations by back-propagating errors. Nature, 323(6088):533-536, 1986.

Carl Runge. Über die numerische auflösung von differentialgleichungen. Mathematische Annalen, 46(2):167-178, 1895.

Simo Sarkka. On sequential monte carlo sampling of discretely observed stochastic differential equations. In 2006 IEEE Nonlinear Statistical Signal Processing Workshop, pages 21-24. IEEE, 2006.

Simo Särkkä. Bayesian filtering and smoothing. Cambridge University Press, 2013.

Simo Särkkä and Arno Solin. Applied stochastic differential equations, volume 10. Cambridge University Press, 2019.

Jürgen Schmidhuber. Deep learning in neural networks: An overview. Neural Networks, 61:85-117, 2015.

Julian Schrittwieser, Ioannis Antonoglou, Thomas Hubert, Karen Simonyan, Laurent Sifre, Simon Schmitt, Arthur Guez, Edward Lockhart, Demis Hassabis, Thore Graepel, et al. Mastering atari, go, chess and shogi by planning with a learned model. Nature, 588(7839):604-609, 2020. 
John Schulman, Sergey Levine, Pieter Abbeel, Michael Jordan, and Philipp Moritz. Trust region policy optimization. In Proceedings of the 32nd International Conference on Machine Learning, pages 1889-1897, 2015.

John Schulman, Philipp Moritz, Sergey Levine, Michael Jordan, and Pieter Abbeel. High-dimensional continuous control using generalized advantage estimation. In International Conference on Learning Representations, 2016.

Matthias Seeger, Christopher K. I. Williams, and Neil D. Lawrence. Fast forward selection to speed up sparse Gaussian process regression. In In Workshop on AI and Statistic, 9, 2003.

Robert H Shumway, David S Stoffer, and David S Stoffer. Time series analysis and its applications, volume 3. Springer, 2000.

Yang Song, Jascha Sohl-Dickstein, Diederik P Kingma, Abhishek Kumar, Stefano Ermon, and Ben Poole. Score-based generative modeling through stochastic differential equations. arXiv preprint arXiv:2011.13456, 2020.

Ilya Sutskever, Geoffrey E Hinton, and Graham W Taylor. The recurrent temporal restricted Boltzmann machine. In Advances in Neural Information Processing Systems, pages 1601-1608, 2009.

Richard S Sutton and Andrew G Barto. Reinforcement learning: An introduction. MIT press, 2018.

Corentin Tallec, Léonard Blier, and Yann Ollivier. Making deep Q-learning methods robust to time discretization. In Proceedings of the 36th International Conference on Machine Learning, 2019.

Yuval Tassa and Tom Erez. Least squares solutions of the HJB equation with neural network value-function approximators. IEEE Transactions on Neural Networks, 18(4):1031-1041, 2007.

Yuval Tassa, Yotam Doron, Alistair Muldal, Tom Erez, Yazhe Li, Diego de Las Casas, David Budden, Abbas Abdolmaleki, Josh Merel, Andrew Lefrancq, et al. Deepmind control suite. arXiv preprint arXiv:1801.00690, 2018.

Morris Tenenbaum. Ordinary differential equations : an elementary textbook for students of mathematics, engineering and the sciences. Dover, New York, 1985.

Michalis Titsias. Variational learning of inducing variables in sparse Gaussian processes. In Proceedings of the Twelth International Conference on Artificial Intelligence and Statistics. PMLR, 2009.

Emanuel Todorov, Tom Erez, and Yuval Tassa. Mujoco: A physics engine for model-based control. In 2012 IEEE / RSJ International Conference on Intelligent Robots and Systems, pages 5026-5033. IEEE, 2012.

James M. Varah. A spline least squares method for numerical parameter estimation in differential equations. SIAM Journal on Scientific Computing, 3(1): 28-46, 1982.

Draguna Vrabie and Frank Lewis. Neural network approach to continuoustime direct adaptive optimal control for partially unknown nonlinear systems. Neural Networks, 22(3):237-246, 2009.

Jack Wang, Aaron Hertzmann, and David M Blei. Gaussian process dynamical models. In Advances in Neural Information Processing Systems, pages 1441$1448,2006$.

Jack M Wang, David J Fleet, and Aaron Hertzmann. Gaussian process dynamical models for human motion. IEEE Transactions on Pattern Analysis and Machine Intelligence, 30(2):283-298, 2008. 
Yali Wang and David Barber. Gaussian processes for Bayesian estimation in ordinary differential equations. Proceedings of the 31st International Conference on Machine Learning, 2014.

Frank Weber, Stefan Theers, Dirk Surmann, Uwe Ligges, and Claus Weihs. Sensitivity analysis of ordinary differential equation models. Technical report, Technische Universität Dortmund, 2018.

Max Welling and Yee Whye Teh. Bayesian learning via stochastic gradient Langevin dynamics. In Proceedings of the 28th International Conference on Machine Learning, pages 681-688, 2011.

Chris Williams, Edwin V Bonilla, and Kian M Chai. Multi-task Gaussian process prediction. Advances in Neural Information Processing Systems, pages 153-160, 2007.

James Wilson, Viacheslav Borovitskiy, Alexander Terenin, Peter Mostowsky, and Marc Deisenroth. Efficiently sampling functions from Gaussian process posteriors. In Proceedings of the 37th International Conference on Machine Learning, pages 10292-10302. PMLR, 2020.

Pan Xu, Jinghui Chen, Difan Zou, and Quanquan Gu. Global convergence of Langevin dynamics based algorithms for nonconvex optimization. In Advances in Neural Information Processing Systems, volume 31, 2018.

Nanyang Ye, Zhanxing Zhu, and Rafal Mantiuk. Langevin dynamics with continuous tempering for training deep neural networks. In Advances in Neural Information Processing Systems, pages 618-626, 2017.

Sixin Zhang, Anna E. Choromanska, and Yann LeCun. Deep learning with elastic averaging SGD. In Advances in Neural Information Processing Systems, pages 685-693, 2015.

Yuchen Zhang, Percy Liang, and Moses Charikar. A hitting time analysis of stochastic gradient Langevin dynamics. In Proceedings of the 2017 Conference on Learning Theory, volume 65, pages 1980-2022, 2017.

Shen-Yi Zhao and Wu-Jun Li. Fast asynchronous parallel stochastic gradient descent: A lock-free approach with convergence guarantee. In Proceedings of the Thirtieth AAAI Conference on Artificial Intelligence, pages 2379-2385, 2016.

Shuxin Zheng, Qi Meng, Taifeng Wang, Wei Chen, Nenghai Yu, Zhi-Ming Ma, and Tie-Yan Liu. Asynchronous stochastic gradient descent with delay compensation. In Proceedings of the 34th International Conference on Machine Learning, volume 70, pages 4120-4129, 2017.

Yaofeng Desmond Zhong and Naomi Leonard. Unsupervised learning of Lagrangian dynamics from images for prediction and control. In Advances in Neural Information Processing Systems, 2020.

Yaofeng Desmond Zhong, Biswadip Dey, and Amit Chakraborty. Symplectic ODEnet: Learning Hamiltonian dynamics with control. In International Conference on Learning Representations, 2020.

Yuanheng Zhu, Dongbin Zhao, and Xiangjun Li. Using reinforcement learning techniques to solve continuous-time non-linear optimal tracking problem without system dynamics. IET Control Theory \& Applications, 10(12):1339-1347, 2016.

Juntang Zhuang, Nicha Dvornek, Xiaoxiao Li, Sekhar Tatikonda, Xenophon Papademetris, and James Duncan. Adaptive checkpoint adjoint method for gradient estimation in neural ode. In Proceedings of the 37th International Conference on Machine Learning, pages 11639-11649. PMLR, 2020. 
Tarmo Äijö and Harri Lähdesmäki. Learning gene regulatory networks from gene expression measurements using non-parametric molecular kinetics. Bioinformatics, 25:2937-2944, 2009. 
